\title{
Variabilidade Extra-Binomial: Uso de Métodos Bayesianos
}

\author{
Juliano José Guimarães Junqueira
}

Orientador: Prof. Dr. Jorge Alberto Achcar

Dissertação apresentada ao Instituto de Ciências Matemáticas e de Computação - ICMC-USP, como parte dos requisitos para obtenção do título de Mestre em Ciências de Computação e Matemática Computacional.

USP - São Carlos

$\mathrm{Março/2001}$ 
A Comissão Julgadora:

Prof. Dr. Jorge Alberto Achcar
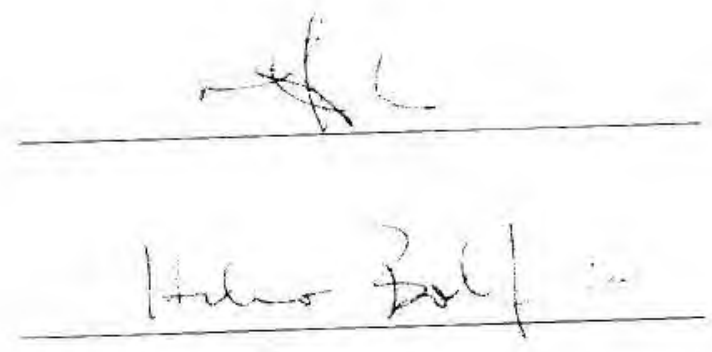

Prof. Dr. Heleno Bolfarine

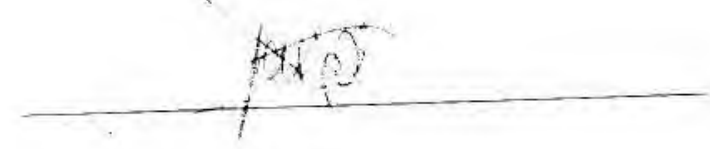

Prof. Dr. Francisco Louzada Neto 


\section{Agradecimentos}

Acima de tudo, agradeço a Deus por me conceder a vida até o presente momento.

Aos meus pais, José e Tânia, aos meus irmãos, Marcelo e Anamélia, e a todos os meus parentes, por acreditarem em mim e estarem sempre ao meu lado.

Ao Prof. Dr. Jorge Alberto Achcar, por conduzir este trabalho com admirável dedicação e por compartilhar comigo a sua "incontestável" experiência.

Ao Prof. Dr. Francisco Louzada Neto e a Profa. Dra. Maria Creusa Bretas Salles pelas valiosas sugestões dadas em meu exame de qualificação, que vieram a contribuir muito com esta dissertação.

Aos professores desta instituição: Prof. Dr. Marinho Gomes de Andrade Filho, Prof. Dr. Josemar Rodrigues e Prof. Dr. Dorival Leão Pinto Junior pela dedicação e empenho ao ensino.

À todos os meus amigos da USP de São Carlos, que direta ou indiretamente contribuíram para que este trabalho fosse realizado. Em especial agradeço a Cillene, Emerson, Guilherme, Juanita, Josenildo, Josmar, Juan, Leonilce, Lucimary, Manuel, Pio, Rubia, Sandra, Silvio, Ulisses, Valéria, Vanderly, Viviane e William.

A todos os funcionários do ICMC-USP.

À todos os meus colegas da UNESP de Presidente Prudente, pela convivência durante os 4 anos de graduação e pela grande amizade que se formou.

À todos os professores da UNESP de Presidente Prudente, pela dedicação ao ensino e por me incentivarem a fazer um curso de Pós-Graduação. 
A CAPES pelo apoio financeiro.

Enfim, gostaria de agradecer a todos que contribuiram para que este trabalho fosse realizado. 


\begin{abstract}
The Binomial distribution is often used for counts $y$ of successes in $n$ repetitions of a random experiment where each result is a success or a failure.

However, in many applications, we can have a larger or smaller variability as expected with the Binomial suposition with parameters $n$ and $p$. This variability is called extra-Binomial variability and it can be obtained from different sources

Some different models are introduced in the literature to fit the extra-Binomial variability. Among these models, we have the Beta-Binomial model, the correlated Binomial model and the mixture of Binomial distributions. In this dissertation, we analyse these models under the Bayesian approach and usin MCMC (Markov Chain Monte Carlo) methods to get the posterior summaries of interest. Other important point in this dissertation: the presence of covariates in the extra-Binomial variability. We illustrate the proposed methodology with same numerical examples. We also introduce some Bayesian discrimination procedures.
\end{abstract}




\section{Resumo}

A distribuição Binomial é freqüentemente usada quando estamos interessados em ajustar dados de contagens de $y$ sucessos em $n$ ensaios de um mesmo experimento aleatório, onde cada ensaio admite duas respostas: sucesso ou fracasso.

Contudo, em muitas aplicações, podemos ter uma variabilidade observada dos dados maior ou menor do que a variabilidade esperada a partir de uma suposição Binomial com parâmetros $n$ e $p$. Essa variabilidade superior ou inferior dos dados observados em relação a variabilidade do modelo Binomial é chamada variabilidade extra-Binomial e ela pode ser provocada por várias fontes.

Alguns modelos tem sido propostos na literatura para ajustar a variabilidade extraBinomial. Entre eles se destacam os modelos Beta-Binomial, Binomial Correlacionado e Mistura de duas distribuições Binomiais. Nesta dissertação, analisamos esses modelos sob o enfoque Bayesiano utilizando os métodos de Monte Carlo em Cadeia de Markov (MCMC). Em particular utilizamos os algoritmos Gibbs Sampling e Metropolis-Hastings para obter estimadores de Monte Carlo das quantidades a posteriori de interesse dos parâmetros. Outro importante objetivo do trabalho é o estudo da variabilidade extra-Binomial na presença de covariáveis. Apresentamos

exemplos com dados reais assumindo os diferentes modelos propostos e uma discriminação dos modelos via Fator de Bayes. 


\section{Sumário}

Capítulo 1: Introdução

1.1 - Alguns modelos para a variabilidade extra-Binomial __________ 3

1.2 - Características dos métodos Bayesianos e alguns objetivos da dissertação. _____ 4

1.3 - Organização da dissertação ___ 6

Capítulo 2: Análise do modelo Beta-Binomial ___ 7

2.1 - Características do modelo ___ 7

2.2 - Análise Clássica do modelo___ 10

2.3 - Análise Bayesiana do modelo ___ 14

Capítulo 3: Análise do modelo Binomial Correlacionado __ 16

3.1 - Características do modelo _ـ 16

3.2 - Análise Clássica do modelo 18

3.3 - Análise Bayesiana do modelo ______ 20

Capítulo 4: Análise do modelo de mistura de duas distribuiçôes Binomiais _ 23

4.1-Características do modelo 23

4.2 - Análise Clássica do modelo 24

4.3- Introdução de variáveis latentes ________________________ 27

4.4 - Análise Bayesiana e algoritmo de simulação

Capítulo 5: Discriminação dos modelos 
5.2 - Discriminação Bayesiana dos modelos: verossimilhança marginal e fator de Bayes ___ 33

5.2 .1 - Estimação da verossimilhança marginal e aplicação nos modelos _ 35

5.2.1.1 - Aproximação de Monte Carlo ___ 35

5.2.1.2 - Método de Laplace ___ _ _ _ _ _ _ _ _ 37

5.2.2 - Aplicação do Fator de Bayes no teste de hipotese. envolvendo os modelos Binomial e Beta-Binomial

Capítulo 6: Variabilidade extra-Binomial na presença de covariáveis 40

6.1 - Modelo de regressão logística 40

6.2 - Modelos para ajustar a variabilidade extra-Binomial na presença de covariáveis 42

6.3 - Análise estatística dos modelos 43

6.3.1 - Análise Clássica dos modelos 44

6.3.2 - Análise Bayesiana dos modelos 51

\section{Capítulo 7: Aplicações Numéricas}

7.1 - Primeira aplicação: dados genéticos 55

7.1.1 - Aplicação do modelo Binomial 56

7.1.2 - Aplicação do modelo Beta-Binomial 57

7.1.3 - Aplicação do modelo Binomial Correlacionado 59

7.1 .4 - Aplicação do modelo de mistura de duas distribuições Binomiais 61

7.1 .5 - Comparação dos modelos 64

7.2 - Segunda aplicação: dados de um jogador de basquete 66

7.2.1 - Resultados da análise Clássica 67

7.2.2 - Resultados da análise Bayesiana 69

7.3 - Terceira aplicação: um exemplo com covariáveis 72

7.3.1 - Aplicação do modelo de Regressão Logistica 74

7.3.2 - Aplicação do modelo Binomial Correlacionado com correlações homogêneas 76

7.3.3 - Aplicação do modelo Binomial Correlacionado com correlações diferentes 80 
Capítulo 8: Conclusões e Considerações Futuras

Apêndice A : Algoritmo Gibbs Sampling

Apêndice B : Algoritmo Metropolis-Hastings 86

Apêndice C : Critério de Convergência de Gelman e Rubin 88

Apêndice D : Análise Clássica e Bayesiana do modelo Binomial 90

Apêndice E : Programa Computacional para o modelo Beta-Binomial 94

Referências Bibliográficas 98 


\section{Capítulo 1: Introdução}

Em diversas situações trabalhamos com experimentos onde as unidades observadas admitem apenas dois resultados possiveis, que são chamados de sucesso e fracasso. Experimentos com essa característica ocorrem em diferentes áreas de interesse. Alguns exemplos:

- em uma pesquisa política, o indivíduo diz se aprova ou não o governo do prefeito de sua cidade

- num laboratório, um animal em teste pode morrer ao receber uma certa dose de veneno ou permanecer vivo.

- um missel pode atingir ou não um alvo estabelecido.

- numa partida de basquete, um jogador ao arremessar a bola para a cesta pode acertar a cesta ou errar o arremesso.

- em um experimento agronômico, podemos verificar se uma determinada semente germina ou não de acordo com algumas condições experimentais (temperatura, umidade, tipo de extrato de raiz, etc.).

Nesses exemplos as sucessivas repetições dos experimentos são conhecidas como provas ou ensaios. Definimos por $X$, a variável aleatória para representar o resultado do i-ésimo ensaio. Como cada ensaio admite apenas duas respostas possíveis, sucesso ou fracasso, a variável $X_{t}$ pode ser codificada pelos valores 0 ou 1. Freqüentemente dizemos que ocorre sucesso quando a variável $X_{t}$ assume o valor 1 e fracasso quando a variável $X$, assume o valor 0 e as 
probabilidades de sucesso e fracasso são dadas respectivamente por $p_{i}$ e $1-p_{2}$. Na prática e comum chamar de sucesso o resultado de interesse do pesquisador, mesmo que seja um evento pouco provável de ocorrer. A variável $X_{i}$ que assume apenas os valores 0 ou 1 , é conhecida como a variável aleatória com distribuição de Bernoulli, onde a função densidade de probabilidade, a média e a variância são dadas por,

$$
\begin{aligned}
& P\left(X_{i}=x_{i}\right)=p_{i}^{x_{i}}\left(1-p_{i}\right)^{1-x_{i}}, \text { onde } x_{i}=0 \text { ou } 1 . \\
& E\left(X_{i}\right)=p_{i} \\
& \operatorname{Var}\left(X_{i}\right)=p_{i}\left(1-p_{i}\right)
\end{aligned}
$$

Muitas vezes estamos interessados em determinar o número de sucessos em $n_{i}$ ensaios de um mesmo experimento aleatório, onde cada ensaio admite só duas respostas: sucesso ou fracasso. Definimos a variável aleatória $Y_{i}=X_{i 1}+X_{i 2} \ldots .+X_{i n_{i}}, i=1, \ldots, N$, para representar o número de sucessos nos $n_{i}$ ensaios, onde $X_{i j}\left(i=1, \ldots, N ; j=1, \ldots, n_{i}\right)$ representa o resultado do j-ésimo ensaio referente a i-ésima contagem de sucessos.

A distribuição Binomial é freqüentemente usada para modelar dados dessa forma, onde o objetivo é a contagem de $y_{i}$ sucessos num total de $n_{i}$ ensaios. Essa distribuição assume que os $n_{i}$ ensaios do experimento são independentes e que as probabilidades de sucesso e de fracasso são as mesmas para cada ensaio. Assim, assumindo uma distribuição Binomial com parâmetros $n_{i}$ e $p$ para a variável aleatória $Y_{i}$ temos,

$$
\begin{aligned}
& P\left(Y_{i}=y_{i}\right)=\left(\begin{array}{l}
n_{i} \\
y_{i}
\end{array}\right) p^{y_{i}}(1-p)^{n_{i} y_{i}}, \text { onde } y_{i}=0,1, \ldots, n_{i} \text { e } i=1, \ldots, N \\
& E\left(Y_{i}\right)=n_{i} p \\
& \operatorname{Var}\left(Y_{i}\right)=n_{i} p(1-p)
\end{aligned}
$$

Contudo, em muitas aplicações podemos ter uma variabilidade observada dos dados maior do que a variabilidade esperada se considerarmos a suposição ordinária de uma distribuição $\operatorname{Binomial}\left(n_{i}, p\right)$ para $Y_{t}, i=1, \ldots, N$. Ou seja, em alguns casos o modelo Binomial assumido pode não ser o melhor modelo para ajustar os dados, pois devido a grande 
variabilidade dos dados observados seria arriscado assumir uma probabilidade constante de sucesso para todos os ensaios e uma independência entre os ensaios. Podemos também encontrar algumas situações onde a variabilidade observada dos dados é menor do que a variabilidade esperada assumindo a distribuição $\operatorname{Binomial}\left(n_{i}, p\right)$ para $Y_{i}$. Neste caso também o modelo Binomial não seria o mais correto no ajuste dos dados. Essa variabilidade superior ou inferior dos dados observados em relação a variabilidade do modelo Binomial é chamada de variabilidade extra-Binomial e ela pode ser provocada por várias fontes (ver Hsiao (1994)).

São muitos os problemas práticos em que aparecem conjuntos de dados com variabilidade extra-Binomial. Um exemplo analisado por vários autores (ver Skellam (1948), Altham (1978) e Ruldolfer (1990) ) envolve dados genéticos, onde o objetivo é a associação secundária de cromossomos num tipo de couve-flor(Brassica). Este exemplo é discutido no capitulo 7 deste trabalho. Um outro exemplo interessante (ver Kupper \& Haseman (1978) ) considera um conjunto de dados num laboratório de animais onde são analisados mortes fetais em 3 grandes grupos de controle de ratos. Investigações prévias provam que o ajuste do modelo Binomial para estes conjuntos de dados não é muito bom, e isso motiva o desenvolvimento de outras estratégias de modelagem para ajustar a variabilidade extra-Binomial.

\section{1 - Alguns modelos para a variabilidade extra-Binomial}

Alguns modelos tem sido propostos na literatura para ajustar a variabilidade extraBinomial. Entre eles se destacam os modelos Beta-Binomial, Binomial Correlacionado e misturas de distribuições Binomiais.

No modelo Beta-Binomial (ver Skellam (1948), Griffths (1973) e Crowder (1978) ) a variabilidade extra-Binomial é originada pela distribuição de probabilidade na probabilidade de sucesso $p$ da distribuição Binomial. A distribuição Beta com parâmetros $\alpha$ e $\beta$ é a distribuição escolhida para $p$ e a função densidade de probabilidade para a variável $Y_{i}$ (número de sucessos em $n_{i}$ ensaios) dado $n_{i}, \alpha$ e $\beta$, é dada pela distribuição Beta-Binomial.

O modelo Binomial Correlacionado (ver Altham (1978) e Kupper \& Hasemam (1978) ) assume que a fonte da variabilidade extra-Binomial é a correlação $\delta$ entre as variáveis binárias $X_{i 1}, X_{i 2}, \ldots, X_{i n_{i}}$ de $Y_{i}$. Kupper \& Haseman (1978), utilizando testes da razão de verossimilhança, comparam os ajustes dos modelos Beta-Binomial e Binomial Correlacionado nos dados 
envolvendo mortes fetais em 3 grupos de ratos e encontram pequenas diferenças nos ajustes dos dois modelos, ao passo que ambos os modelos se comportam melhor do que o modelo considerando a distribuição Binomial ordinária.

Uma terceira possibilidade de modelagem para ajustar a variabilidade extra-Binomial é considerar que a contagem de sucessos $y_{i}$ é supostamente gerada por uma mistura finita de distribuições Binomiais (ver Hsiao, 1994). Ou seja, a observação $y_{i}$ origina-se de uma entre $J$ categorias mas a fonte exata é desconhecida.

Neste trabalho, esses modelos são analisados sob o enfoque Bayesiano que é uma área que esta cada vez mais ganhando seu espaço na literatura. A seção seguinte nos mostra as características dos métodos Bayesianos e alguns tópicos importantes considerados nesta dissertação.

\section{2 - Características dos métodos Bayesianos e alguns objetivos da dissertação.}

Métodos Bayesianos são métodos inferenciais alternativos ao métodos tradicionais que podem melhorar a precisão dos resultados. A principal diferença entre os métodos Clássicos e Bayesianos é que os métodos Bayesianos permitem incorporar ao modelo, informações prévias a respeito de um parâmetro de interesse para a análise. Ao contrário da inferência Clássica a inferência Bayesiana considera os parâmetros como variáveis aleatórias com distribuições de probabilidade chamadas de distribuições a priori, disponiveis para melhorar a precisão da inferência. Podemos determinar uma distribuição a priori de uma maneira subjetiva ou através do conhecimento prévio de um especialista da área de interesse. A escolha de uma distribuição a priori adequada é fundamental para garantir a qualidade da inferência Bayesiana

Após estipular as distribuições a priori para os parâmetros do modelo, combinamos estas distribuições com a função de verossimilhança e através da fórmula de Bayes encontramos a distribuição a posteriori conjunta para os parâmetros. Na análise Bayesiana toda a inferência de interesse será baseada nesta distribuição.

Uma vantagem da inferência Bayesiana em relação à inferência Clássica, é que quando temos um grande número de parâmetros envolvidos num modelo, o uso de métodos clássicos torna-se intratável, pois é extremamente trabalhoso a obtenção de estimadores de máxima 
verossimilhança e requer resoluções de equações que podem não ter solução analítica. Procedimentos Bayesianos evitam a necessidade dessas soluções numéricas através do uso dos métodos de Monte Carlo em cadeia de Markov (MCMC). Em particular, utilizamos neste trabalho os algoritmos Gibbs Sampling (ver por exemplo, Gelfand \& Smith (1990) ) e Metropolis-Hastings (ver por exemplo, Chib \& Greenberg (1995) ou Smith \& Roberts (1993) ) para obter estimadores de Monte Carlo das quantidades a posteriori de interesse dos parâmetros.

O algoritmo Gibbs Sampling é um método de simulação via cadeias de Markov, que obtém amostras de uma distribuição de interesse, baseada nas distribuições condicionais a posteriori dos parâmetros (ver apêndice A). Esse algoritmo é utilizado quando as distribuições condicionais apresentam a forma de uma distribuição conhecida.

Em modelos mais complexos as distribuições condicionais a posteriori não são facilmente identificadas como tendo uma forma conhecida, impossibilitando a geração direta das amostras a partir dessas distribuições. Neste caso usamos o algoritmo Metropolis-Hastings para a geração das amostras (ver apêndice B).

Um tópico de grande importância que será analisado neste trabalho é o diagnóstico de convergência das cadeias de Markov. Existem vários métodos para verificação da convergência e um dos mais utilizados é o método de Gelman \& Rubin (ver Gelman \& Rubin, 1992). A idéia deste método é que iniciando com diferentes pontos iniciais, as trajetórias de várias cadeias devem ser similares se convergirem para uma distribuição estacionária. Gelman \& Rubin (1992) formalizam essas idéias através do uso das técnicas de análise de variâncias (ver apêndice C). Neste trabalho, utilizamos este método para a verificação da convergência em todos os modelos usados para ajustar a variabilidade extra-Binomial.

Outro ponto importante do trabalho que abordamos na análise Bayesiana, é o estudo da variabilidade extra-Binomial na presença de covariáveis (ver Williams (1982) ). Um modelo que é freqüentemente usado para dados de contagens de sucessos, quando trabalhamos com covariáveis, é o modelo de regressão logística. Contudo, em algumas situações, esse modelo pode não se ajustar bem aos dados e um dos motivos está relacionado a presença da variabilidade extra-Binomial. Neste caso, consideramos outras estratégias de modelagem e desenvolvemos uma análise Bayesiana para esses modelos baseada nos métodos MCMC.

Para alguns modelos realizamos uma análise clássica, através da determinação de estimadores de máxima verossimilhança para os paràmetros e comparamos os resultados com aqueles obtidos sob o enfoque Bayesiano. 
Também, apresentamos exemplos com dados reais assumindo os diferentes modelos propostos e uma discriminação dos modelos seguindo alguns critérios que são discutidos no decorrer da dissertação.

\section{3 - Organização da dissertação}

Esta dissertação está dividida em 8 capitulos.

Neste capitulo inicial, discutimos o problema relacionado a variabilidade extra-Binomial, quando o nosso interesse é a contagem de sucessos dos ensaios de um experimento. Introduzimos alguns modelos usados para ajustar essa variabilidade e apresentamos as principais características dos métodos Bayesianos, utilizados no decorrer do trabalho.

Nos capítulos 2, 3 e 4, apresentamos uma análise estatística dos modelos usados para ajustar a variabilidade extra-Binomial, introduzidos na seção 1.1. Analisamos o modelo BetaBinomial no capitulo 2, o modelo Binomial Correlacionado no capítulo 3 e o modelo de mistura de duas distribuições Binomiais no capitulo 4.

Com o objetivo de verificar qual desses modelos é o mais apropriado para ajustar os dados e se o ajuste desses modelos é superior ao ajuste do modelo Binomial, apresentamos no capitulo 5 dois critérios usados para discriminar os modelos.

O capitulo 6 é dedicado ao estudo da variabilidade extra-Binomial na presença de covariàveis.

No capitulo 7, analisamos conjuntos de dados reais para ilustrar a metodologia proposta nos capitulos anteriores.

Para finalizar, no capitulo 8 apresentamos as conclusões do trabalho e sugerimos algumas considerações futuras. 


\section{Capítulo 2: Análise do modelo Beta-Binomial}

Neste capitulo, apresentamos uma análise estatística detalhada do modelo Beta-Binomial, utilizando os métodos Clássicos e Bayesianos. Inicialmente, na seção 3.1, apresentamos algumas caracteristicas do modelo, o desenvolvimento matemático para obter a expressão da densidade Beta-Binomial e comentamos sobre uma reparametrização $(\xi, \omega)$ que tem sido usada por alguns autores. $\mathrm{Na}$ análise Clássica do modelo descrita na seção 3.2 , introduzimos o procedimento para encontrar os estimadores de máxima verossimilhança utilizando o método iterativo de NewtonRaphson. $\mathrm{Na}$ análise Bayesiana do modelo descrita na seção 3.3, assumimos diferentes distribuições a priori para os parâmetros e encontramos as distribuições condicionais necessárias para o uso dos métodos de Monte Carlo em Cadeia de Markov (MCMC).

Continuando com a notação introduzida no capítulo 1, vamos considerar neste e nos demais capítulos a variável aleatória $Y_{i}$ para representar o número de sucessos em $n_{i}$ ensaios, onde cada ensaio admite duas respostas: sucesso ou fracasso.

\section{1 - Características do modelo}

Este modelo foi proposto por Skellam (1948) que apresenta expressões para os momentos fatoriais da distribuição Beta-Binomial e descreve a estimação dos paràmetros pelo método dos momentos e pelo método de máxima verossimilhança.

Consideremos a suposição usual dada no capítulo anterior onde,

$$
Y_{i} / n_{i}, p \sim \operatorname{Binomial}\left(n_{i}, p\right), i=1, \ldots, N
$$

Uma possibilidade para ajustar os dados com variabilidade extra-Binomial é assumir uma distribuição de probabilidade sobre o parâmetro $p$ da distribuição Binomial. No modelo BetaBinomial assumimos uma distribuição Beta para $p$ com parâmetros $\alpha$ e $\beta$. Essa distribuição 
tem sido usada por muitos autores (ver por exemplo, Griffiths (1973) and Crowder (1978)) pela sua simplicidade matemática. Assim, temos,

$$
f(p / \alpha, \beta)=\frac{\Gamma(\alpha+\beta)}{\Gamma(\alpha) \Gamma(\beta)} p^{\alpha-1}(1-p)^{\beta-1}, \quad 0 \leq p \leq 1, \quad \alpha>0 \text { e } \beta>0
$$

Desta forma, a função densidade de probabilidade da variável $Y_{1}, i=1, \ldots, N$, dado os parâmetros $n_{i}, \alpha$ e $\beta$, pode ser calculada por,

$$
\begin{aligned}
& f\left(y_{i} / n_{i}, \alpha, \beta\right)=\int_{i}^{1} f\left(y_{i}, p / n_{i}, \alpha, \beta\right) d p \\
& =\int_{0}^{1} f\left(y_{i} / p, n_{i}, \alpha, \beta\right) f\left(p / n_{i}, \alpha, \beta\right) d p \\
& =\int_{i}^{1}\left(\begin{array}{l}
n_{i} \\
y_{i}
\end{array}\right) p^{y_{i}}(1-p)^{n_{i}-y_{i}} \frac{\Gamma(\alpha+\beta)}{\Gamma(\alpha) \Gamma(\beta)} p^{\alpha-1}(1-p)^{\beta-1} d p
\end{aligned}
$$

Como, $\int_{0}^{1} p^{\alpha-1}(1-p)^{\beta-1} d p=\frac{\Gamma(\alpha) \Gamma(\beta)}{\Gamma(\alpha+\beta)}$

temos,

$$
\begin{aligned}
& f\left(y_{i} / n_{i}, \alpha, \beta\right)=\left(\begin{array}{l}
n_{i} \\
y_{i}
\end{array}\right) \frac{\Gamma(\alpha+\beta)}{\Gamma(\alpha) \Gamma(\beta)} \frac{\Gamma\left(\alpha+y_{i}\right) \Gamma\left(\beta+n_{i}-y_{i}\right)}{\Gamma\left(\alpha+\beta+n_{i}\right)} \\
& =\left(\begin{array}{l}
n_{i} \\
y_{i}
\end{array}\right) \frac{B\left(\alpha+y_{i}, \beta+n_{i}-y_{i}\right)}{B(\alpha, \beta)}
\end{aligned}
$$

onde: $B(\alpha, \beta)=\frac{\Gamma(\alpha) \Gamma(\beta)}{\Gamma(\alpha+\beta)}$

A função densidade da variável $Y_{i}$ dada em (2.4), é conhecida como a densidade da distribuição Beta-Binomial. A esperança e a variância de $Y_{i}, i=1, \ldots ., N$, são dadas por, 


$$
\begin{aligned}
& E\left(Y_{i} / n_{i}, \alpha, \beta\right)=\frac{n_{i} \alpha}{\alpha+\beta} \\
& \operatorname{Var}\left(Y_{t} / n_{i}, \alpha, \beta\right)=n_{i}\left(\frac{\alpha}{\alpha+\beta}\right)\left(1-\frac{\alpha}{\alpha+\beta}\right)\left(\frac{n_{i}+\alpha+\beta}{1+\alpha+\beta}\right)
\end{aligned}
$$

Observando a expressão $\operatorname{Var}\left(Y_{t} / n_{i}, \alpha, \beta\right)$ dada em (2.5), percebemos que o termo $\left(\frac{n_{i}+\alpha+\beta}{1+\alpha+\beta}\right)$ é sempre maior ou igual do que 1 , pois $\alpha>0, \beta>0$ e $n_{i} \geq 1$. Este fato torna a variância do modelo Beta-Binomial sempre maior ou igual do que a variância do modelo Binomial, o que permite ajustar dados com variabilidade superior a da distribuição Binomial ordinária (ver Rudolfer, 1990).

Uma parametrização usada por muitos autores (ver por exemplo, Williams (1982) e Tarone (1979) ) considera:

$$
\begin{aligned}
& \xi=\frac{\alpha}{\alpha+\beta} \\
& \omega=\frac{1}{\alpha+\beta}
\end{aligned}
$$

Com esta reparametrização $(\xi, \omega)$, as expressões (2.4) e (2.5) podem ser reescritas como:

$$
\begin{aligned}
& f\left(y_{i} / n_{i}, \alpha, \beta\right)=\left(\begin{array}{l}
n_{i} \\
y_{i}
\end{array}\right) \frac{\prod_{j=0}^{y_{i}-1}(\alpha+j) \prod_{j=0}^{n_{i}-y_{i}-1}(\beta+j)}{\prod_{j=0}^{n_{i}: 1}(\alpha+\beta+j)}=\left(\begin{array}{l}
n_{i} \\
y_{i}
\end{array}\right) \frac{\prod_{j=0}^{y_{i}-1}(\xi+\omega j) \prod_{j=0}^{n_{i}-y_{i}-1}(1-\xi+\omega j)}{\prod_{j=0}^{n_{i}-1}(1+\omega j)} \\
& E\left(Y_{i} / n_{i}, \xi\right)=n_{i} \xi \\
& \operatorname{Var}\left(Y_{i} / n_{i}, \xi, \omega\right)=n_{i} \xi(1-\xi) \frac{n_{i}+\omega^{1}}{1+\omega^{1}}
\end{aligned}
$$


Uma vantagem de usar os parâmetros $\xi$ e $\omega$ é que podemos verificar que a distribuição Beta-Binomial tende suavemente para a distribuição Binomial quando $\omega \rightarrow 0$. Assim, existe uma ligação entre os modelos Binomial e Beta-Binomial através do parâmetro $\omega$ e este parâmetro pode ser usado para selecionar qual o melhor modelo a partir do teste de hipótese,

$$
\begin{aligned}
& H_{0}: \omega=0 \text { (distribuição Binomial) } \\
& H_{1}: \omega>0 \text { (distribuição Beta-Binomial) }
\end{aligned}
$$

No capitulo 5, que é o capítulo dedicado à discriminação dos modelos, discutimos em detalhes esse teste de hipóteses.

\section{2 - Análise Clássica do modelo}

A abordagem clássica do modelo Beta-Binomial e de outros modelos é feita com base na função de verossimilhança que expressa toda a informação contida no conjunto de dados de tamanho $N$. Assumindo que as contagens de sucessos $y_{1}, y_{2}, \ldots, y_{N}$ são independentes, a função de verossimilhança para o modelo Beta-Binomial, utilizando a expressão (2.4), é dada por,

$$
\begin{aligned}
& L(\alpha, \beta)=\prod_{i=1}^{N}\left[\left(\begin{array}{l}
n_{i} \\
y_{i}
\end{array}\right) \frac{\Gamma(\alpha+\beta)}{\Gamma(\alpha) \Gamma(\beta)} \frac{\Gamma\left(\alpha+y_{i}\right) \Gamma\left(\beta+n_{i}-y_{i}\right)}{\Gamma\left(\alpha+\beta+n_{i}\right)}\right] \\
& =\prod_{i=1}^{N}\left[\left(\begin{array}{l}
n_{i} \\
y_{i}
\end{array}\right) \frac{\prod_{j=0}^{y_{i}-1}(\alpha+j) \prod_{j-0}^{n_{i}-y_{i}-1}(\beta+j)}{\prod_{j=0}^{n_{i}-1}(\alpha+\beta+j)}\right]
\end{aligned}
$$

$\mathrm{O}$ nosso objetivo é encontrar valores para os parâmetros $\alpha$ e $\beta$ que maximizem a função de verossimilhança. Esses valores são conhecidos como estimadores de máxima 
verossimilhança (EMV) de $\alpha$ e $\beta$ e são os valores dos parâmetros mais prováveis de gerar os dados observados. Matematicamente è mais fácil trabalhar com o logaritmo da função de verossimilhança. O logaritmo da expressão (2.9) é dado por,

$$
\begin{aligned}
& l(\alpha, \beta)=\log [L(\alpha, \beta)]
\end{aligned}
$$

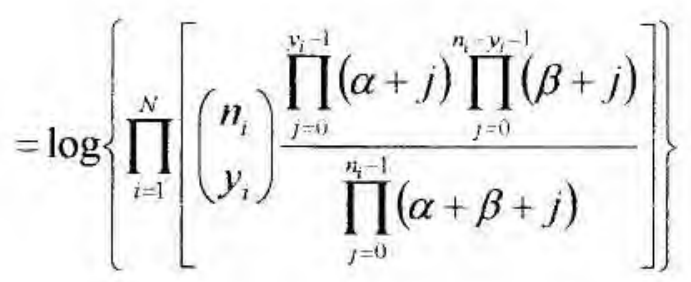

$$
\begin{aligned}
& =\sum_{i=1}^{N}\left\{\log \left[\left(\begin{array}{l}
n_{i} \\
y_{i}
\end{array}\right) \frac{\prod_{j=1}^{y_{j}-1}(\alpha+j) \prod_{j=0}^{n_{i}-y_{i}-1}(\beta+j)}{\prod_{j=0}^{n_{i}-1}(\alpha+\beta+j)}\right]\right\} \\
& =\sum_{i=1}^{N}\left[\log \left(\begin{array}{l}
n_{i} \\
y_{i}
\end{array}\right)\right]+\sum_{i=1}^{N}\left\{\log \left[\prod_{j=0}^{y_{i}-1}(\alpha+j)\right]\right\}+\sum_{i=1}^{N}\left\{\log \left[\prod_{j=1}^{n_{i}-y_{i}-1}(\beta+j)\right]\right\}-\sum_{i=1}^{N}\left\{\log \left[\prod_{j=0}^{n_{i}-1}(\alpha+\beta+j)\right]\right\} \\
& =\sum_{i=1}^{N}\left[\log \left(\begin{array}{l}
n_{i} \\
y_{i}
\end{array}\right)\right]+\sum_{i=1}^{N} \sum_{j=0}^{y_{j}-1}[\log (\alpha+j)]+\sum_{i=1}^{N} \sum_{j=1}^{n_{i}-y_{i}-1}[\log (\beta+j)]-\sum_{i=1}^{N} \sum_{j=1}^{n_{i}-1}[\log (\alpha+\beta+j)]
\end{aligned}
$$

Derivando o logaritmo da função de verossimilhança em relação aos parâmetros $\alpha$ e $\beta$ temos:

$$
\begin{aligned}
& \frac{\partial l(\alpha, \beta)}{\partial \alpha}=\sum_{i=1}^{N} \sum_{j=0}^{y_{j}-1}\left(\frac{1}{\alpha+j}\right)-\sum_{j=1}^{N} \sum_{j=0}^{n_{i}-1}\left(\frac{1}{\alpha+\beta+j}\right) \\
& \frac{\partial l(\alpha, \beta)}{\partial \beta}=\sum_{i=1}^{N} \sum_{j=0}^{n_{i}-y_{i}-1}\left(\frac{1}{\beta+j}\right)-\sum_{i=1}^{N} \sum_{j=0}^{n_{i}-1}\left(\frac{1}{\alpha+\beta+j}\right)
\end{aligned}
$$


Os EMV de $\alpha$ e $\beta$ podem ser determinados de (2.11), encontrando os valores de $\alpha$ e $\beta$ que são soluções das equações:

$$
\begin{aligned}
& \sum_{i=1}^{N} \sum_{j=0}^{y_{j}-1}\left(\frac{1}{\alpha+j}\right)-\sum_{i=1}^{N} \sum_{j=0}^{n_{j}-1}\left(\frac{1}{\alpha+\beta+j}\right)=0 \\
& \sum_{i=1}^{N} \sum_{j=0}^{n_{j}} \sum_{j}^{\prime}\left(\frac{1}{\beta+j}\right)-\sum_{i=1}^{N} \sum_{j=1}^{n_{i}-1}\left(\frac{1}{\alpha+\beta+j}\right)=0
\end{aligned}
$$

Como as equações dadas em (2.12) não são lineares em relação aos parâmetros $\alpha$ e $\beta$, precisamos utilizar algum método numérico para encontrar suas soluções. Um método prático muito utilizado é o método de Newton-Raphson (ver por exemplo, Lee (1992)). Este método tem um procedimento iterativo, onde $\alpha_{k}$ e $\beta_{k}$ denotam as estimativas de $\alpha$ e $\beta$ na k-ésima iteração e os valores para a $(k+1)$-ésima iteração são obtidos da seguinte forma:

$$
\left(\begin{array}{l}
\hat{\alpha}_{(k+1)} \\
\hat{\beta}_{(k+1)}
\end{array}\right)=\left(\begin{array}{l}
\hat{\alpha}_{(k)} \\
\hat{\beta}_{(k)}
\end{array}\right)+\left(I\left(\hat{\alpha}_{(k)}, \hat{\beta}_{(k)}\right)^{-1} U\left(\hat{\alpha}_{(k)}, \hat{\beta}_{(k)}\right)\right)
$$

onde: $U\left(\alpha(k), \beta_{(k)}\right)=\left(\frac{\frac{\partial l(\alpha, \beta)}{\partial \alpha}}{\frac{\partial l(\alpha, \beta)}{\partial \beta}}\right)_{(\alpha, \beta)}$ é chamado de vetor escore

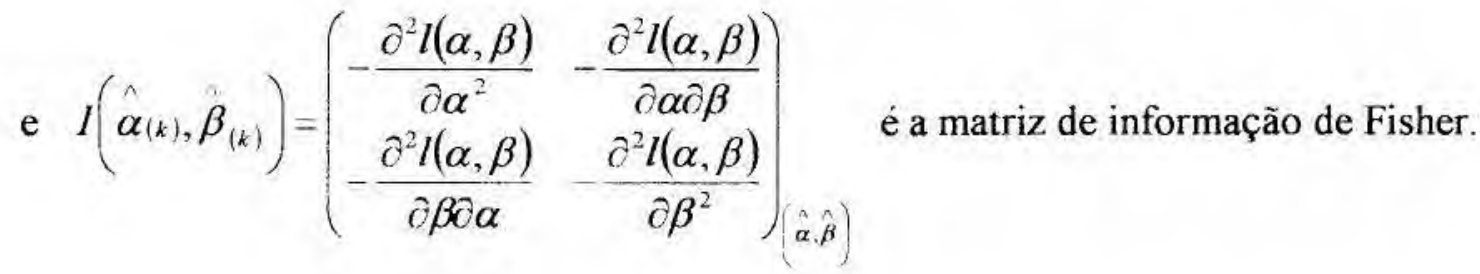


As segundas derivadas do logaritmo da função de verossimilhança em relação aos parâmetros $\alpha$ e $\beta$ são dadas por,

$$
\begin{aligned}
& \frac{\partial^{2} l(\alpha, \beta)}{\partial \alpha^{2}}=-\sum_{i=1}^{N} \sum_{j=0}^{y_{j}-1}\left(\frac{1}{\alpha+j}\right)^{2}+\sum_{i=1}^{N} \sum_{j=0}^{n_{i}-1}\left(\frac{1}{\alpha+\beta+j}\right)^{2} \\
& \frac{\partial^{2} l(\alpha, \beta)}{\partial \beta^{2}}=-\sum_{i=1}^{N} \sum_{j=1}^{n_{i}-y_{i}-1}\left(\frac{1}{\beta+j}\right)^{2}+\sum_{i=1}^{N} \sum_{j=0}^{n_{i}-1}\left(\frac{1}{\alpha+\beta+j}\right)^{2} \\
& \frac{\partial^{2} l(\alpha, \beta)}{\partial \alpha \partial \beta}=\frac{\partial^{2} l(\alpha, \beta)}{\partial \beta \partial \alpha}=\sum_{i=1}^{N} \sum_{j=0}^{n_{i}-1}\left(\frac{1}{\alpha+\beta+j}\right)^{2}
\end{aligned}
$$

$O$ processo iterativo termina quando as diferenças nos valores de $\alpha$ e $\beta$ em duas iterações consecutivas for desprezivel. É fundamental darmos estimativas iniciais adequadas para $\alpha$ e $\beta$, para garantir a convergência dos parâmetros em poucas iterações. Podemos ter, problemas com a aplicação do método de Newton-Raphson em situações onde temos um grande número de parâmetros para serem estimados, o que poderá levar a uma sobrecarga computacional, dificultando a convergência.

No passo final das iterações, além de obtermos estimativas para os parâmetros $\alpha$ e $\beta$, conseguimos também uma estimativa da matriz de variância-covariância de $\alpha$ e $\beta$, utilizando a propriedade assintótica dos estimadores de máxima verossimilhança. Esta propriedade diz que: se $\hat{\alpha}$ e $\beta$ são EMV de $\alpha$ e $\beta$, então $(\hat{\alpha}, \hat{\beta})^{a} \sim N\left((\alpha, \beta), I^{1}\right)$, onde $I$ é a matriz de informação de Fisher. Desta forma, podemos determinar intervalos de confiança assintóticos para os parâmetros. 


\section{3 - Análise Bayesiana do modelo}

Nesta seção, analisamos o modelo Beta-Binomial sob o enfoque Bayesiano. Para isso consideramos os parâmetros $\alpha$ e $\beta$ como variáveis aleatórias, ou seja assumimos distribuições de probabilidade a priori para esses parâmetros. Para encontrar amostras das distribuições a posteriori marginais de $\alpha$ e $\beta$, utilizamos os métodos de Monte Carlo em Cadeia de Markov (MCMC), introduzidos no capitulo 1. Com essas amostras obtidas por simulação, podemos estimar várias características dos parâmetros como a média e o desvio padrão, além de encontrar intervalos de credibilidade para os mesmos.

Assumimos as seguintes distribuições a priori para os parâmetros:

(i) $\alpha \sim \Gamma\left(a_{1}, b_{1}\right) ; \quad a_{1}, b_{1}$ conhecidos

(ii) $\beta \sim \Gamma\left(a_{2}, b_{2}\right) ; \quad a_{2}, b_{2}$ conhecidos

onde $\Gamma(a, b)$ denota uma distribuição Gama com parâmetros $a$ e $b$.

Considerando que as distribuições a priori para os parâmetros são independentes, a função densidade conjunta a priori para $\alpha$ e $\beta$ é dada por,

$$
\pi(\alpha, \beta) \propto \alpha^{a_{1}-1} e^{b_{1} \alpha} \beta^{a_{2}} e^{b_{2} \beta}
$$

Combinando (2.16) com a função de verossimilhança do modelo Beta-Binomial dada em (2.9), encontramos a densidade a posteriori conjunta para $\alpha$ e $\beta$ dada por,

$$
\pi(\alpha, \beta / y, n) \propto \alpha^{a_{1}-1} e^{-b_{1} \alpha} \beta^{a_{2}-1} e^{b_{2} \beta}\left\{\frac{\Gamma(\alpha+\beta)}{\Gamma(\alpha) \Gamma(\beta)}\right\}^{N} \prod_{i=1}^{N}\left[\frac{\Gamma\left(\alpha+y_{i}\right) \Gamma\left(\beta+n_{i}-y_{i}\right)}{\Gamma\left(\alpha+\beta+n_{i}\right)}\right]
$$

onde: $y=\left(y_{1}, \ldots, y_{N}\right)$ e $n=\left(n_{1}, \ldots, n_{N}\right)$. 
A partir de (2.17), encontramos as distribuições condicionais a posteriori de $\alpha$ e $\beta$, que são necessárias para o uso dos métodos MCMC. Essas distribuições são dadas por,

(i) $\pi(\alpha / \beta, y, n) \propto \alpha^{\alpha_{1}-1} e^{b_{1} \alpha}\left\{\frac{\Gamma(\alpha+\beta)}{\Gamma(\alpha)}\right\}^{N} \prod_{i=1}^{N}\left[\frac{\Gamma\left(\alpha+y_{i}\right)}{\Gamma\left(\alpha+\beta+n_{i}\right)}\right]$
$\propto \alpha^{a_{1}-1} e^{b_{1} \alpha} \exp \left\{N \log [\Gamma(\alpha+\beta)]-N \log [\Gamma(\alpha)]+\sum_{i=1}^{N} \log \left[\Gamma\left(\alpha+y_{i}\right)\right]-\sum_{i=1}^{N} \log \left[\Gamma\left(\alpha+\beta+n_{i}\right)\right]\right\}$

ou $\pi(\alpha / \beta, y, n) \propto \Gamma\left(a_{1}, b_{1}\right) \psi_{1}(\alpha, \beta)$

onde:

$\psi_{1}(\alpha, \beta)=\exp \left\{N \log [\Gamma(\alpha+\beta)]-N \log [\Gamma(\alpha)]+\sum_{i=1}^{N} \log \left[\Gamma\left(\alpha+y_{i}\right)\right]-\sum_{i=1}^{N} \log \left[\Gamma\left(\alpha+\beta+n_{i}\right)\right]\right\}$

(ii) $\pi(\beta / \alpha, y, n) \propto \beta^{a_{2}-1} e^{-b_{2} \beta}\left\{\frac{\Gamma(\alpha+\beta)}{\Gamma(\beta)}\right\}^{N} \prod_{i=1}^{N}\left[\frac{\Gamma\left(\beta+n_{i}-y_{i}\right)}{\Gamma\left(\alpha+\beta+n_{i}\right)}\right]$

$\propto \beta^{a_{2}-1} e^{-b_{2} \beta} \exp \left\{N \log [\Gamma(\alpha+\beta)]-N \log [\Gamma(\beta)]+\sum_{i=1}^{N} \log \left[\Gamma\left(\beta+n_{i}-y_{i}\right)\right]-\sum_{i=1}^{N} \log \left[\Gamma\left(\alpha+\beta+n_{i}\right)\right]\right\}$

ou $\pi\left(\beta / \alpha, y_{2} n\right) \propto \Gamma\left(a_{2}, b_{2}\right) \psi_{2}(\alpha, \beta)$

onde:

$\psi_{2}(\alpha, \beta)=\exp \left\{N \log [\Gamma(\alpha+\beta)]-N \log [\Gamma(\beta)]+\sum_{i=1}^{N} \log \left[\Gamma\left(\beta+n_{i}-y_{i}\right)\right]-\sum_{i=1}^{N} \log \left[\Gamma\left(\alpha+\beta+n_{i}\right)\right]\right\}$

Como as distribuições condicionais para $\alpha$ e $\beta$ não apresentam formas de distribuições conhecidas, utilizamos o algoritmo Metropolis-Hastings para realizar a simulação dos parâmetros e fazer as inferências desejadas. 


\section{Capítulo 3: Análise do modelo}

\section{Binomial Correlacionado}

Neste capitulo, analisamos o modelo Binomial Correlacionado introduzido por Altham (1978) e Kupper \& Haseman (1978). Na seção 3.1, apresentamos as idéias básicas do modelo, as expressões da função densidade, da média e da variância e as diferenças desse modelo em relação ao modelo Beta-Binomial. Nas seções 3.2 e 3.3 , analisamos o modelo Binomial Correlacionado através dos métodos Clássicos e Bayesianos, respectivamente, utilizando os mesmos procedimentos empregados na análise do modelo Beta-Binomial.

\section{1 - Características do modelo}

Como já comentamos no capitulo 1, umas das suposições necessárias ao assumir o modelo Binomial para a variável $Y_{i}=X_{i 1}+X_{i 2}+\ldots . .+X_{i n_{i}}$, que representa o número de sucessos em $n_{i}$ ensaios, é considerar que os $n_{t}$ ensaios são independentes, ou seja o resultado de um ensaio não tem influência no resultado de outro ensaio. Contudo, Altham (1978) e Kupper \& Haseman (1978) trabalhando com experimentos toxicológicos em laboratórios de animais, analisam dados de contagem de sucessos onde o modelo Binomial não se ajusta muito bem. Para resolver este problema, eles propõem o modelo Binomial Correlacionado que assume uma correlação entre as variáveis binárias $X_{i 1}, X_{i 2}, \ldots, X_{i n}$ de $Y_{1}$. Neste caso, a variabilidade extraBinomial é provocada por esta correlação. Este modelo surge como uma alternativa ao modelo Beta-Binomial, analisado no capítulo 2, que já existia na literatura.

Representamos por $\delta$ a correlação entre os pares de variáveis binárias de $Y_{i}, i=1, \ldots, N$. Esta correlação, chamada de correlação intra-classe, é suposta homogênea entre $Y_{1}, Y_{2}, \ldots, Y_{N}$.

Como $X_{i j} \sim \operatorname{Bernoulli}(p)$, temos, 


$$
\begin{aligned}
& \delta=\operatorname{Corr}\left(X_{i j}, X_{i k}\right)=\frac{\operatorname{Cov}\left(X_{i j}, X_{i k}\right)}{\sqrt{\operatorname{Var}\left(X_{i}\right) \operatorname{Var}\left(X_{i k}\right)}}= \\
& =\frac{E\left(X_{i j} X_{i k}\right)-E\left(X_{i j}\right) E\left(X_{i k}\right)}{\sqrt{\operatorname{Var}\left(X_{i j}\right) \operatorname{Var}\left(X_{i k}\right)}}=\frac{P\left(X_{i j}=1, X_{i k}=1\right)-p^{2}}{p(1-p)} \\
& \mathrm{p} / \mathrm{j} \neq k, \quad j, k=1, \ldots, n_{i}, i=1, \ldots, N .
\end{aligned}
$$

A função densidade de probabilidade da variável $Y_{i}, i=1, \ldots, N$, dado $n_{i}, \delta$ e $p$, é dada por,

$$
f\left(y_{i} / n_{i}, \delta, p\right)=\left(\begin{array}{l}
n_{i} \\
y_{i}
\end{array}\right) p^{y_{i}}(1-p)^{n_{i}-y_{i}}\left\{1+\frac{\delta}{2 p(1-p)}\left[\left(y_{i}-n_{i} p\right)^{2}+y_{i}(2 p-1)-n_{i} p^{2}\right]\right\}
$$

A esperança e a variância de $Y_{i}, i=1, \ldots, N$, são dadas por,

$$
\begin{aligned}
& E\left(Y_{i} / n_{i}, \delta, p\right)=n_{1} p \\
& \operatorname{Var}\left(Y_{i} / n_{i}, \delta, p\right)=n_{i} p(1-p)\left[1+\left(n_{i}-1\right) \delta\right] \Rightarrow \frac{-1}{n_{i}-1}<\delta \leq 1
\end{aligned}
$$

Uma propriedade do modelo Binomial Correlacionado que não é apresentada pelo modelo Beta-Binomial, é que além de ajustar conjuntos de dados com una variabilidade superior a variabilidade do modelo Binomial, permite também ajustar dados com uma dispersão inferior a da distribuição Binomial ordinária. Este fato é possivel pois a correlação $\delta$ pode assumir valores negativos, o que faz com que o termo $\left[1+\left(n_{i}-1\right) \delta\right]$ de $\operatorname{Var}\left(Y_{i}\right)$, dado em (3.3), assuma valores entre 0 e 1. Quando isto ocorre, a variância do modelo Binomial Correlacionado é menor do que variância do modelo Binomial (ver Prentice, 1986). Todavia, conjuntos de dados com essa dispersão inferior não são muito comuns e na maioria dos problemas envolvendo variabilidade extra-Binomial predominam a alta dispersão dos dados. 


\section{2 - Análise Clássica do modelo}

Nesta seção, analisamos o modelo Binomial Correlacionado sob o ponto de vista clássico, apresentando as expressões necessárias para a obtenção dos estimadores de máxima verossimilhança dos parâmetros $\delta$ e $p$.

Considerando independentes as contagens de sucessos $y_{1}, y_{2}, \ldots, y_{N}$, a função de verossimilhança para o modelo Binomial Correlacionado, utilizando a expressão (3.2), é dada por,

$$
\begin{aligned}
& L(\delta, p)=\prod_{i=1}^{N} f\left(y_{i} / n_{i}, \delta, p\right)= \\
& =\prod_{i=1}^{N}\left[\left(\begin{array}{l}
n_{i} \\
y_{i}
\end{array}\right) p^{y_{i}}(1-p)^{n_{i}-y_{i}}\left\{1+\frac{\delta}{2 p(1-p)}\left[\left(y_{i}-n_{i} p\right)^{2}+y_{i}(2 p-1)-n_{i} p^{2}\right]\right\}\right]
\end{aligned}
$$

Aplicando o logaritmo na expressão (3.4) temos,

$$
\begin{aligned}
& l(\delta, p)=\log [I(\delta, p)] \\
& =\log \left\{\prod_{i}^{N}\left[\left(\begin{array}{l}
n_{i} \\
y_{i}
\end{array}\right) p^{y_{i}}(1-p)^{n_{i}-y_{i}}\left\{1+\frac{\delta}{2 p(1-p)}\left[\left(y_{i}-n_{i} p\right)^{2}+y_{i}(2 p-1)-n_{i} p^{2}\right]\right\}\right]\right\} \\
& =\sum_{i=1}^{N}\left\{\log \left[\left(\begin{array}{l}
n_{i} \\
y_{i}
\end{array}\right) p^{y_{i}}(1-p)^{n_{i}-y_{i}}\left\{1+\frac{\delta}{2 p(1-p)}\left[\left(y_{i}-n_{i} p\right)^{2}+y_{i}(2 p-1)-n_{i} p^{2}\right]\right\}\right]\right\} \\
& =\sum_{i=1}^{N}\left[\log \left(\begin{array}{l}
n_{i} \\
y_{i}
\end{array}\right)\right]+\log (p) \sum_{i=1}^{N} y_{i}+\log (1-p) \sum_{i=1}^{N}\left(n_{i}-y_{i}\right) \\
& +\sum_{i=1}^{N}\left\{\log \left[1+\frac{\delta}{2 p(1-p)}\left[\left(y_{i}-n_{i} p\right)^{2}+y_{i}(2 p-1)-n_{i} p^{2}\right]\right]\right\}
\end{aligned}
$$




$$
\begin{aligned}
& =\sum_{i=1}^{N}\left[\log \left(\begin{array}{l}
n_{i} \\
y_{i}
\end{array}\right)\right]+\log (p) \sum_{i=1}^{N} y_{i}+\log (1-p) \sum_{i=1}^{N}\left(n_{i}-y_{i}\right) \\
& +\sum_{i=1}^{N}\left\{\operatorname { l o g } \left[2 p(1-p)+\delta\left[\left(y_{i}-n_{i} p\right)^{2}+y_{i}(2 p-1)-n_{i} p^{2} \mathbb{W}-\sum_{i=1}^{N}\{\log [2 p(1-p)]\}\right.\right.\right.
\end{aligned}
$$

As derivadas de primeira ordem do logaritmo da função de verossimilhança em relação aos parâmetros $\delta$ e $p$ são dadas por,

$$
\begin{aligned}
& \frac{\partial l(\delta, p)}{\partial \delta}=\sum_{i=1}^{N}\left[\frac{\left(y_{i}-n_{i} p\right)^{2}+y_{i}(2 p-1)-n_{i} p^{2}}{2 p(1-p)+\delta\left[\left(y_{i}-n_{i} p\right)^{2}+y_{i}(2 p-1)-n_{i} p^{2}\right]}\right] \\
& \frac{\partial l(\delta, p)}{\partial p}=\frac{\sum_{i=1}^{N} y_{i}}{p}-\frac{\sum_{i=1}^{N}\left(n_{i}-y_{i}\right)}{1-p} \\
& +\sum_{i=1}^{N}\left[\frac{2(1-2 p)+\delta\left[-2 n_{i}\left(y_{i}-n_{i} p\right)+2 y_{i}-2 n_{i} p\right]}{2 p(1-p)+\delta\left[\left(y_{i}-n_{i} p\right)^{2}+y_{i}(2 p-1)-n_{i} p^{2}\right.}\right]-\sum_{i=1}^{N}\left(\frac{1-2 p}{p(1-p)}\right)
\end{aligned}
$$

Igualando as expressões dadas em (3.6) a 0 e resolvendo o sistema de equações, determinamos os EMV de $\delta$ e $p$. Como estas equações não são lineares em relação aos parâmetros, utilizamos também neste modelo o método iterativo de Newton-Raphson para encontrar suas soluções, seguindo o mesmo procedimento descrito no capítulo anterior.

As derivadas de segunda ordem do logaritmo da função de verossimilhança em relação a $\delta$ e $p$, necessárias para o método de Newton-Raphson, são dadas por,

$$
\left.\frac{\partial^{2} l(\delta, p)}{\partial \delta^{2}}=-\sum_{i=1}^{N}\left[\frac{\left(y_{i}-n_{i} p\right)^{2}+y_{i}(2 p-1)-n_{i} p^{2}}{2 p(1-p)+\delta\left(y_{i}-n_{i} p\right)^{2}+y_{i}(2 p-1)-n_{i} p^{2}}\right]\right]^{2}
$$




$$
\begin{aligned}
& \frac{\partial^{2} l(\delta, p)}{\partial p^{2}}=-\frac{\sum_{i=1}^{N} y_{i}}{p^{2}}+\frac{\sum_{i=1}^{N}\left(n_{i}-y_{i}\right)}{(1-p)^{2}} \\
& +\sum_{i=1}^{N}\left[\frac{\left(-4+\delta\left[2 n_{i}^{2}-2 n_{i}\right)\right.}{2 p(1-p)+\delta\left[\left(y_{i}-n_{i} p\right)^{2}+y_{i}(2 p-1)-n_{i} p^{2}\right]}\right] \\
& -\sum_{i=1}^{N}\left[\frac{\left(2(1-2 p)+\delta\left[-2 n_{i}\left(y_{i}-n_{i} p\right)+2\left(y_{i}-n_{i} p\right)\right]^{2}\right.}{\left(2 p(1-p)+\delta\left[\left(y_{i}-n_{i} p\right)^{2}+y_{i}(2 p-1)-n_{i} p^{2}\right)^{2}\right.}\right]-\sum_{i=1}^{N}\left(\frac{-2 p(1-p)-(1-2 p)^{2}}{[p(1-p)]^{2}}\right) \\
& \frac{\partial^{2} l(\delta, p)}{\partial \delta \partial p}=\frac{\partial^{2} l(\delta, p)}{\partial p \partial \delta} \\
& =\sum_{i=1}^{N}\left[\frac{-2 n_{i}\left(y_{i}-n_{i} p\right)+2\left(y_{i}-n_{i} p\right)}{2 p(1-p)+\delta\left[\left(y_{i}-n_{i} p\right)^{2}+y_{i}(2 p-1)-n_{i} p^{2}\right]}\right] \\
& -\sum_{i=1}^{N}\left[\frac{\left(2(1-2 p)+\delta\left(-2 n_{i}\left(y_{i}-n_{i} p\right)+2\left(y_{i}-n_{i} p\right)\right)\right)\left(\left(y_{i}-n_{i} p\right)^{2}+y_{i}(2 p-1)-n_{i} p^{2}\right)}{\left(2 p(1-p)+\delta\left[\left(y_{i}-n_{i} p\right)^{2}+y_{i}(2 p-1)-n_{i} p^{2}\right)^{2}\right.}\right]
\end{aligned}
$$

\section{3 - Análise Bayesiana do modelo}

Realizamos uma análise Bayesiana para o modelo Binomial Correlacionado com base na escolha das distribuições a priori para os parâmetros $\delta$ e $p$. Consideramos 2 possibilidades: começamos assumindo uma distribuição a priori informativa para a probabilidade de sucesso $p$ e uma distribuição a priori não informativa para a correlação $\delta$. Posteriormente, assumimos distribuições a priori não informativas para os dois parâmetros. Em ambas as situações encontramos as distribuições condicionais a posteriori para $\delta$ e $p$, necessárias para o uso dos métodos MCMC.

Considerando a primeira estratégia, as distribuiçoes a priori escolhidas para os parâmetros são dadas por, 
(i) $\delta \sim U(a, b) ; a, b$ conhecidos

(ii) $p \sim \operatorname{Beta}(c, d) ; c, d$ conhecidos

Assumindo independência a priori para os parâmetros, a função densidade conjunta a priori para $\delta$ e $p$ é dada por,

$$
\pi(\delta, p) \propto p^{c 1}(1-p)^{d-1}
$$

Combinando as expressões (3.4) e (3.9), encontramos a densidade a posteriori conjunta para $\delta$ e $p$ dada por,

$$
\begin{gathered}
\pi(\delta, p / y, n) \propto p^{c+\sum_{i=1}^{N} v}(1-p)^{d+\sum_{i=1}^{N}\left(n_{i}-y_{i}+1\right.} \prod_{i=1}^{N}\left\{1+\frac{\delta}{2 p(1-p)}\left[\left(y_{i}-n_{i} p\right)^{2}+y_{i}(2 p-1)-n_{i} p^{2}\right]\right\} \\
\text { onde: } y=\left(y_{1}, \ldots, y_{N}\right) \text { e } n=\left(n_{1}, \ldots, n_{N}\right) .
\end{gathered}
$$

Portanto, as distribuições condicionais a posteriori para os parâmetros $\delta$ e $p$ são dadas por,

(i)

$$
\begin{aligned}
& \pi(\delta / p, y, n) \propto \prod_{i=1}^{N}\left\{1+\frac{\delta}{2 p(1-p)}\left[\left(y_{i}-n_{i} p\right)^{2}+y_{i}(2 p-1)-n_{i} p^{2}\right]\right\} \\
& \text { ou } \pi(\delta / p, y, n) \propto U(a, b) \psi(\delta, p)
\end{aligned}
$$

(ii)

$$
\begin{aligned}
& \pi(p / \delta, y, n) \propto p^{c+\sum_{i=1}^{N} y_{i}-1}(1-p)^{d+\sum_{i=1}^{N}\left(n_{i}-y_{i}\right)+1} \prod_{i=1}^{N}\left\{1+\frac{\delta}{2 p(1-p)}\left[\left(y_{i}-n_{i} p\right)^{2}+y_{i}(2 p-1)-n_{i} p^{2}\right]\right\} \\
& \text { ou } \pi(p / \delta, y, n) \propto \operatorname{Beta}\left(c+\sum_{i=1}^{N} y_{i}, d+\sum_{i=1}^{N}\left(n_{i}-y_{i}\right)\right) \psi(\delta, p) \\
& \text { onde: } \psi(\delta, p) \propto \prod_{i=1}^{N}\left\{1+\frac{\delta}{2 p(1-p)}\left[\left(y_{i}-n_{i} p\right)^{2}+y_{i}(2 p-1)-n_{i} p^{2}\right]\right\}
\end{aligned}
$$


Considerando a segunda estratégia, assumimos as seguintes distribuições a priori não informativas para os parâmetros:

(i) $\delta \sim U(a, b) ; a, b$ conhecidos

(ii) $p \sim U(c, d) ; c, d$ conhecidos

Assumindo que as distribuiçōes a priori para os parâmetros são independentes, temos que a função densidade conjunta a priori è uma constante em relação a $\delta$ e $p$. Desta forma a distribuição a posteriori conjunta para $\delta$ e $p$ é proporcional a função de verossimilhança dada em (3.4), ou seja:

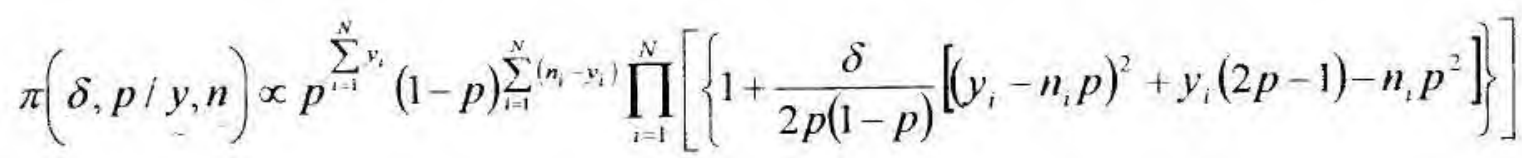

Desta forma, encontramos as distribuições condicionais a posteriori para os parâmetros $\delta$ e $p$. Para $\delta$ a distribuição condicional é a mesma expressão dada em (3.11) e para o parâmetro $p$ a distribuição condicional é dada por,

$$
\begin{aligned}
& \pi(p / \delta, y, n) \propto p^{\sum_{i=1}}(1-p)_{i=1}^{\sum_{i}\left(n_{i}-y_{i}\right)} \prod_{i=1}^{N}\left\{1+\frac{\delta}{2 p(1-p)}\left[\left(y_{i}-n_{i} p\right)^{2}+y_{i}(2 p-1)-n_{i} p^{2}\right]\right\} \\
& \text { ou } \pi(p / \delta, y, n) \propto \operatorname{Beta}\left(\sum_{i=1}^{N} y_{i}+1, \sum_{i=1}^{N}\left(n_{i}-y_{i}\right)+1\right) \psi(\delta, p) \\
& \text { onde: } \psi(\delta, p) \text { é dado em (3.11). }
\end{aligned}
$$

Observamos que as distribuições condicionais a posteriori para os parâmetros dadas em (3.11) e (3.14) não apresentam formas de distribuições conhecidas. Portanto, em ambos os casos utilizamos o algoritmo Metropolis-Hastings para realizar a simulação dos parâmetros e fazer as inferências desejadas. 


\section{Capítulo 4: Análise do modelo de mistura de duas distribuições Binomiais}

Os modelos baseados em distribuições mistas, formam uma classe de modelos estatísticos que são naturalmente aplicados quando as observações amostrais são obtidas em diferentes situações, isto é quando temos uma população heterogênea (ver Titterington et.al. (1985) ). A análise Bayesiana desses modelos está sendo recentemente utilizada em diversas áreas com grande sucesso, devido a formulação de novos algoritmos computacionais que facilitam a estimação dos parâmetros.

Este capitulo apresenta uma análise Clássica e Bayesiana para o modelo de mistura de duas distribuições Binomiais. Na seção 4.1, definimos o modelo geral de mistura de $J$ distribuições Binomiais e o caso particular considerando mistura de duas Binomiais. Na seção 4.2, apresentamos uma análise Clássica para o modelo de mistura de duas distribuições Binomiais. Na seção 43, introduzimos variáveis latentes para simplificar a função de verossimilhança desse modelo. Utilizando esta função de verossimilhança simplificada, desenvolvemos na seção 4.4 uma análise Bayesiana para o modelo, encontrando as distribuições condicionais necessárias para os métodos MCMC, e apresentamos o algoritmo de simulação baseado na introdução das variáveis latentes.

\section{1 - Características do modelo}

A distribuição Beta-Binomial discutida no capítulo 2, assume uma distribuição continua $F_{p}($ ) sobre o parâmetro $p$, a probabilidade Binomial. Uma outra possibilidade para ajustar a variabilidade extra-Binomial, é considerar $F$ degenerada num número finito de pontos. Em outras palavras, consideramos que a observação $y_{i}$ é supostamente gerada por uma mistura finita de $J$ distribuições Binomiais com probabilidades de sucesso diferentes dadas por $\theta_{1}, \theta_{2}, \ldots, \theta_{J}$. Neste caso, a função densidade de probabilidade para a variável $Y_{i},(i=1, \ldots, N)$, é dada por, 


$$
f\left(y_{i} / n_{i}, \theta_{1}, \ldots, \theta_{J}, \pi_{1}, \ldots, \pi_{J}\right)=\sum_{j=1}^{J} \pi_{j}\left(\begin{array}{l}
n_{i} \\
y_{i}
\end{array}\right) \theta_{j}^{y_{j}}\left(1-\theta_{j}\right)^{n_{i} y_{i}}
$$

onde $\pi_{j},(j=1, \ldots, J)$, denota a probabilidade de que a observação $y_{i}$ foi gerada da jésima distribuição Binomial e $\sum_{j=1}^{J} \pi_{j}=1$.

Desta forma, $y_{i}$ é originada de uma entre $J$ categorias, mas a fonte exata é desconhecida

Considerando o caso especial de mistura de duas distribuições Binomiais, onde $J=2$, a distribuição de $Y_{i},(i=1, \ldots ., N)$, é dada por,

$$
\begin{aligned}
& Y_{i} / n_{i}, \theta_{1}, \theta_{2}, \pi \sim \pi \operatorname{Binomial}\left(n_{i}, \theta_{1}\right)+(1-\pi) \operatorname{Binomial}\left(n_{i}, \theta_{2}\right) \\
& \Rightarrow f\left(y_{i} / n_{i}, \theta_{1}, \theta_{2}, \pi\right)=\pi\left(\begin{array}{l}
n_{i} \\
y_{i}
\end{array}\right) \theta_{1}^{y_{i}}\left(1-\theta_{1}\right)^{n_{i}-y_{i}}+(1-\pi)\left(\begin{array}{l}
n_{i} \\
y_{i}
\end{array}\right) \theta_{2}^{y_{i}}\left(1-\theta_{2}\right)^{n_{i}-y_{i}}
\end{aligned}
$$

onde $\pi$ denota a probabilidade de que $y_{i}$ foi gerada da primeira distribuição Binomial com probabilidade de sucesso $\theta_{1}$ e $1-\pi$ denota a probabilidade de que $y_{t}$ foi gerada da segunda distribuição Binomial com probabilidade de sucesso $\theta_{2}$.

\section{2 - Análise Clássica do modelo}

Nesta seção, analisamos o modelo de mistura de duas distribuições Binomiais sob o ponto de vista clássico, apresentando as expressões necessárias para a obtenção dos estimadores de máxima verossimilhança dos parâmetros $\theta_{1}, \theta_{2}$ e $\pi$.

Assumindo que as contagens de sucessos $y_{1}, y_{2}, \ldots, y_{N}$ são independentes, a função de verossimilhança para o modelo de mistura de duas distribuições Binomiais, utilizando a expressão (4.2), é dada por, 


$$
L\left(\theta_{1}, \theta_{2}, \pi\right)=\prod_{i=1}^{N}\left\{\pi\left(\begin{array}{l}
n_{t} \\
y_{i}
\end{array}\right) \theta_{1}^{y_{i}}\left(1-\theta_{1}\right)^{n_{i} y_{i}}+(1-\pi)\left(\begin{array}{l}
n_{i} \\
y_{i}
\end{array}\right) \theta_{2}^{y_{i}}\left(1-\theta_{2}\right)^{n_{i}-y_{i}}\right\}
$$

Aplicando o logaritmo na expressão (4.3), temos:

$$
\begin{aligned}
& l\left(\theta_{1}, \theta_{2}, \pi\right)=\log \left[L\left(\theta_{1}, \theta_{2}, \pi\right)\right] \\
& =\log \left\{\prod_{i=1}^{N}\left[\pi\left(\begin{array}{l}
n_{i} \\
y_{i}
\end{array}\right) \theta_{1}^{y_{i}}\left(1-\theta_{1}\right)^{n_{i}-y_{i}}+(1-\pi)\left(\begin{array}{l}
n_{i} \\
y_{i}
\end{array}\right) \theta_{2}^{y_{i}}\left(1-\theta_{2}\right)^{n_{i}-y_{i}}\right]\right\} \\
& =\sum_{i=1}^{N}\left\{\log \left[\pi\left(\begin{array}{l}
n_{i} \\
y_{i}
\end{array}\right) \theta_{i}^{y_{i}}\left(1-\theta_{1}\right)^{n_{i}-y_{i}}+(1-\pi)\left(\begin{array}{l}
n_{i} \\
y_{i}
\end{array}\right) \theta_{2}^{y_{i}}\left(1-\theta_{2}\right)^{n_{i}-y_{i}}\right]\right\}
\end{aligned}
$$

As derivadas de primeira ordem do logaritmo da função de verossimilhança em relação aos parâmetros $\theta_{1}, \theta_{2}$ e $\pi$ são dadas por,

$$
\begin{aligned}
& \frac{\partial l\left(\theta_{1}, \theta_{2}, \pi\right)}{\partial \theta_{1}}=\sum_{i=1}^{N} \pi\left[\frac{y_{i} \theta_{1}^{y_{i}-1}\left(1-\theta_{1}\right)^{n_{i}-y_{i}}-\left(n_{i}-y_{i}\right) \theta_{1}^{y_{i}}\left(1-\theta_{1}\right)^{n_{i}-y_{i}-1}}{\pi \theta_{1}^{y_{i}}\left(1-\theta_{1}\right)^{n_{i}-y_{i}}+(1-\pi) \theta_{2}^{y_{i}}\left(1-\theta_{2}\right)^{n_{i}} y_{i}}\right] \\
& \frac{\partial l\left(\theta_{1}, \theta_{2}, \pi\right)}{\partial \theta_{2}}=\sum_{i=1}^{N}(1-\pi)\left[\frac{y_{i} \theta_{2}^{y_{i}-1}\left(1-\theta_{2}\right)^{n_{i}-y_{i}}-\left(n_{i}-y_{i}\right) \theta_{2}^{y_{i}}\left(1-\theta_{2}\right)^{n_{i}-y_{i}} !}{\pi \theta_{1}^{y_{i}}\left(1-\theta_{1}\right)^{n_{i} y_{i}}+(1-\pi) \theta_{2}^{y_{i}}\left(1-\theta_{2}\right)^{n_{i}-y_{i}}}\right] \\
& \frac{\partial l\left(\theta_{1}, \theta_{2}, \pi\right)}{\partial \pi}=\sum_{i=1}^{N}\left[\frac{\theta_{1}^{y_{i}}\left(1-\theta_{1}\right)^{n_{i}-y_{i}}-\theta_{2}^{y_{i}}\left(1-\theta_{2}\right)^{n_{i}-y_{i}}}{\pi \theta_{1}^{y_{i}}\left(1-\theta_{1}\right)^{n_{i}-y_{i}}+(1-\pi) \theta_{2}^{y_{i}}\left(1-\theta_{2}\right)^{n_{i}-y_{i}}}\right]
\end{aligned}
$$

Igualando as expressões dadas em (4.5) a 0 e resolvendo o sistema de equações, determinamos os EMV de $\theta_{1}, \theta_{2}$ e $\pi$. Como estas equações não são lineares em relação aos 
parâmetros, utilizamos também neste modelo o método iterativo de Newton-Raphson para encontrar suas soluções, seguindo o mesmo procedimento descrito no capitulo 2 .

As derivadas de segunda ordem do logaritmo da função de verossimilhança em relação a $\theta_{1}, \theta_{2}$ e $\pi$, necessárias para o método de Newton-Raphson, são dadas por,

$$
\begin{aligned}
& \frac{\partial^{2} l\left(\theta_{1}, \theta_{2}, \pi\right)}{\partial \theta_{1}^{2}}=\sum_{i=1}^{N} \pi\left[\frac{y_{i}\left(y_{i}-1\right) \theta_{1}^{y_{i}-2}\left(1-\theta_{1}\right)^{n_{2}-y_{i}}-2 y_{i}\left(n_{i}-y_{i}\right) \theta_{1}^{y_{i}-1}\left(1-\theta_{1}\right)^{n_{i}-y_{i}-1}+\left(n_{i}-y_{i}\right)\left(n_{i}-y_{i}-1\right) \theta_{1}^{y_{i}}\left(1-\theta_{1}\right)^{n_{i}-y_{i}-2}}{\pi \theta_{i}\left(1-\theta_{1}\right)^{n_{i}-y_{i}}+(1-\pi) \theta_{2}^{y_{i}}\left(1-\theta_{2}\right)^{n_{i}-y_{i}}}\right] \\
& -\sum_{i=1}^{N} \pi\left[\left(\frac{y_{i} \theta_{1}^{y_{i}-1}\left(1-\theta_{1}\right)^{n_{i}-y_{i}}-\left(n_{i}-y_{i}\right) \theta_{1}^{y_{i}}\left(1-\theta_{1}\right)^{n_{i}-y_{i}-1}}{\pi \theta_{1}^{y_{i}}\left(1-\theta_{1}\right)^{n_{i}-y_{i}}+(1-\pi) \theta_{2}^{y_{i}}\left(1-\theta_{2}\right)^{n_{i}-y_{i}}}\right)^{2}\right]
\end{aligned}
$$$$
\frac{\partial^{2} l\left(\theta_{1}, \theta_{2}, \pi\right)}{\partial \theta_{2}^{2}}=\sum_{i=1}^{N}(1-\pi)\left[\frac{y_{i}\left(y_{i}-1\right) \theta_{2}^{y_{i}-2}\left(1-\theta_{2}\right)^{n_{i}-y_{i}}-2 y_{i}\left(n_{i}-y_{i}\right) \theta_{2}^{y_{i}-1}\left(1-\theta_{2}\right)^{n_{i}-y_{i}-1}+\left(n_{i}-y_{i}\right)\left(n_{i}-y_{i}-1\right) \theta_{2}^{y_{i}}\left(1-\theta_{2}\right)^{n_{-}-y_{i}-2}}{\pi \theta_{1}^{y_{i}}\left(1-\theta_{1}\right)^{n_{i}-y_{i}}+(1-\pi) \theta_{2}^{y_{i}}\left(1-\theta_{2}\right)^{n_{i}-y_{i}}}\right]
$$$$
-\sum_{i=1}^{N}(1-\pi)\left[\left(\frac{y_{i} \theta_{2}^{y_{i}-1}\left(1-\theta_{2}\right)^{n_{i}-y_{i}}-\left(n_{i}-y_{i}\right) \theta_{2}^{y_{i}}\left(1-\theta_{2}\right)^{n_{i}-y_{i}-1}}{\pi \theta_{1}^{y_{i}}\left(1-\theta_{1}\right)^{n_{i}-y_{i}}+(1-\pi) \theta_{2}^{y_{i}}\left(1-\theta_{2}\right)^{n_{i}-y_{i}}}\right)^{2}\right]
$$$$
\frac{\partial^{2} l\left(\theta_{1}, \theta_{2}, \pi\right)}{\partial \pi^{2}}=-\sum_{i=1}^{N}\left[\left(\frac{\theta_{1}^{y_{i}}\left(1-\theta_{1}\right)^{n_{i}-y_{i}}-\theta_{2}^{y_{i}}\left(1-\theta_{2}\right)^{n_{i}-y_{i}}}{\pi \theta_{1}^{y_{i}}\left(1-\theta_{1}\right)^{n_{i}-y_{i}}+(1-\pi) \theta_{2}^{y_{i}}\left(1-\theta_{2}\right)^{n_{i} y_{i}}}\right)^{2}\right]
$$

$\frac{\partial^{2}\left(\theta_{1}, \theta_{2}, \pi\right)}{\partial \theta_{1} \partial \theta_{2}}=-\sum_{i=1}^{N} \pi(1-\pi)\left[\frac{\left(y_{i} \theta_{1}^{y_{i}-1}\left(1-\theta_{1}\right)^{n^{n-y_{i}}}-\left(n_{i}-y_{i}\right) \theta_{1}^{y_{i}}\left(1-\theta_{1}\right)^{n_{-}-y_{i}-1}\right)\left(y_{i} \theta_{2}^{y_{i}-1}\left(1-\theta_{2}\right)^{n_{-}-y_{i}}-\left(n_{i}-y_{i}\right) \theta_{2}^{y_{i}}\left(1-\theta_{2}\right)^{n_{i}-y_{i}-1}\right)}{\left(\pi \theta_{i}\left(1-\theta_{1}\right)^{n_{-}-y_{i}}+(1-\pi) \theta_{2}^{y_{i}}\left(1-\theta_{2}\right)^{n_{-}-y_{i}}\right)^{2}}\right]$

$$
\begin{aligned}
& \frac{\partial^{2} l\left(\theta_{1}, \theta_{2}, \pi\right)}{\partial \theta_{1} \partial \theta_{\pi}}=-\sum_{i=1}^{N} \pi\left[\frac{\left(y_{i} \theta_{1}^{y_{i}-1}\left(1-\theta_{1}\right)^{n_{2}-y_{i}}-\left(n_{i}-y_{i}\right) \theta_{1}^{y_{i}}\left(1-\theta_{1}\right)^{n_{i}-y_{i}-1}\right)\left(\theta_{1}^{y_{i}}\left(1-\theta_{1}\right)^{n_{i}-y_{i}}-\theta_{2}^{y_{i}}\left(1-\theta_{2}\right)^{n_{i} y_{i}}\right)}{\left(\pi \theta_{1}^{y_{i}}\left(1-\theta_{1}\right)^{n_{i} y_{i}}+(1-\pi) \theta_{2}^{y_{i}}\left(1-\theta_{2}\right)^{n_{i} y_{i}}\right)^{2}}\right] \\
& \frac{\partial^{2} l\left(\theta_{1}, \theta_{2}, \pi\right)}{\partial \theta_{2} \partial \theta_{\pi}}=-\sum_{i=1}^{N}(1-\pi)\left[\frac{\left(y_{i} \theta_{2}^{y_{i}-1}\left(1-\theta_{2}\right)^{n_{i}-y_{i}}-\left(n_{i}-y_{i}\right) \theta_{2}^{y_{i}}\left(1-\theta_{2}\right)^{n_{i}-y_{i}-1}\right)\left(\theta_{1}^{y_{i}}\left(1-\theta_{1}\right)^{n_{i}-y_{i}}-\theta_{2}^{y_{i}}\left(1-\theta_{2}\right)^{n_{i}-y_{i}}\right)}{\left(\pi \theta_{1}^{y_{i}}\left(1-\theta_{1}\right)^{n_{i}-y_{i}}+(1-\pi) \theta_{2}^{y_{i}}\left(1-\theta_{2}\right)^{n_{i}-y_{i}}\right)^{2}}\right]
\end{aligned}
$$




\section{3- Introdução de variáveis latentes}

Como podemos observar, a função de verossimilhança dada em (4.3), apesar de englobar uma mistura de apenas duas distribuições Binomiais, é uma expressão bastante complexa pois envolve somas de termos. Este fato, na análise Bayesiana, acaba dificuitando o trabalho computacional quando utilizamos os métodos MCMC, independente da escolha das distribuições a priori. Para simplificar este problema, introduzimos no modelo variáveis não observáveis $Z_{i}$, $(i=1, \ldots, N)$, também chamadas de variáveis latentes, que acabam simplificando o algoritmo de simulação dos métodos MCMC (ver por exemplo, Tanner \& Wong (1987)) .

A variável latente $Z_{i},(i=1, \ldots, N)$, é definida como:

$Z_{t}=\left\{\begin{array}{l}1 \text { se a } \mathrm{i} \text { - ésima observação foi gerada da primeira distribuição Binomial } \\ 0 \text { se a } \mathrm{i} \text { - ésima observação foi gerada da segunda distribuição Binomial }\end{array}\right.$

ou seja, $Z_{i}$ tem uma distribuição de Bernoulli com probabilidade de sucesso dada por,

$$
h_{i}=P\left(Z_{i}=1 / y_{i}, n_{i}, \theta_{1}, \theta_{2}, \pi\right)=\frac{\pi\left(\begin{array}{l}
\boldsymbol{n}_{i} \\
y_{i}
\end{array}\right) \theta_{1}^{y_{i}}\left(1-\theta_{1}\right)^{n_{i}-y_{i}}}{\pi\left(\begin{array}{l}
n_{i} \\
y_{i}
\end{array}\right) \theta_{1}^{y_{i}}\left(1-\theta_{1}\right)^{n_{i}-y_{i}}+(1-\pi)\left(\begin{array}{l}
\boldsymbol{n}_{i} \\
y_{i}
\end{array}\right) \theta_{2}^{y_{i}}\left(1-\theta_{2}\right)^{n_{i}-y_{i}}}
$$

Desta forma, temos,

$$
\begin{aligned}
& f\left(z_{i} / y_{i}, n_{i}, \theta_{1}, \theta_{2}, \pi\right)=h_{i}^{z_{i}}\left(1-h_{i}\right)^{1-z_{i}}
\end{aligned}
$$

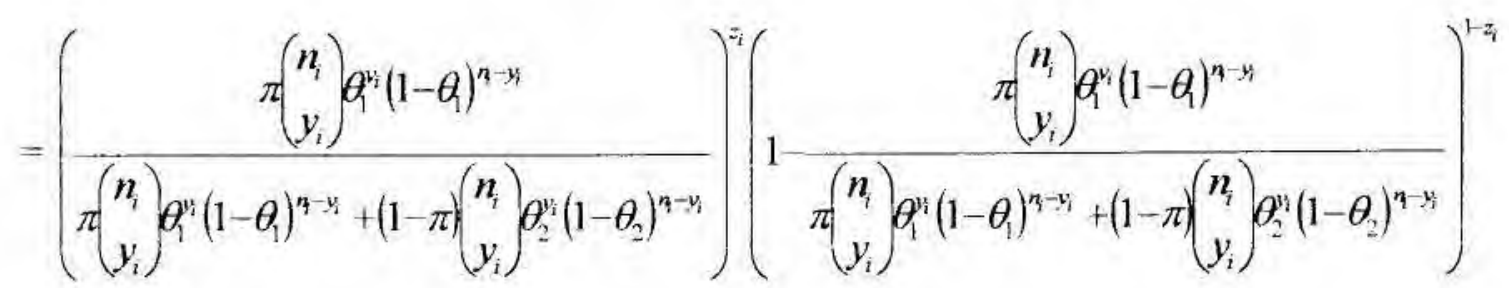




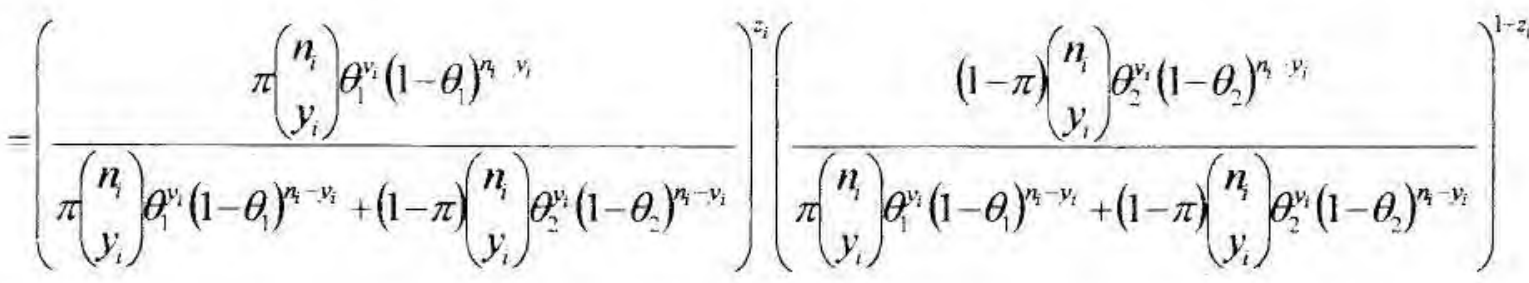

$$
\begin{aligned}
& \frac{\left(\pi\left(\begin{array}{l}
n_{i} \\
y_{i}
\end{array}\right) \theta_{i}^{y_{i}}\left(1-\theta_{1}\right)^{n_{i}-v_{i}}\right)^{z_{i}}\left((1-\pi)\left(\begin{array}{l}
n_{i} \\
y_{i}
\end{array}\right) \theta_{2}^{y_{i}}\left(1-\theta_{2}\right)^{n_{i}-y_{i}}\right)^{1-z_{i}}}{\pi\left(\begin{array}{l}
n_{i} \\
y_{i}
\end{array}\right) \theta_{1}^{y_{i}}\left(1-\theta_{1}\right)^{n_{i}-y_{i}}+(1-\pi)\left(\begin{array}{l}
n_{i} \\
y_{i}
\end{array}\right) \theta_{2}^{y_{i}}\left(1-\theta_{2}\right)^{n_{i}-y_{i}}}
\end{aligned}
$$

Isto é,

$$
\begin{aligned}
& \pi\left(z / n, y, \theta_{1}, \theta_{2}, \pi\right)=\prod_{i=1}^{N} f\left(z_{i} / y_{i}, n_{i}, \theta_{1}, \theta_{2}, \pi\right)
\end{aligned}
$$

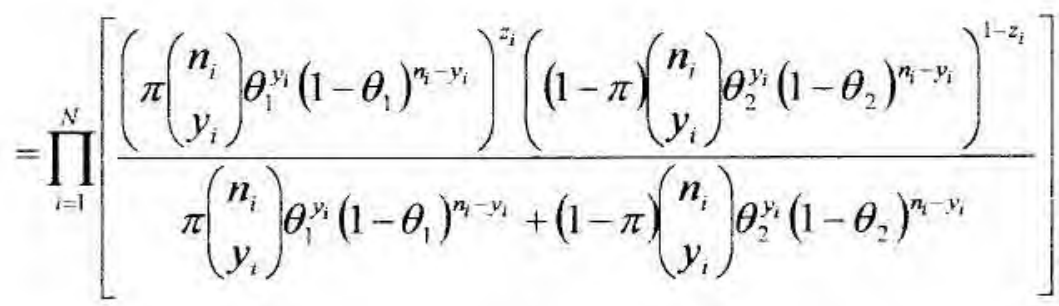

$$
\begin{aligned}
& =\frac{\left.\prod_{i=1}^{N}\left[\left(\begin{array}{l}
\pi \\
n_{i} \\
y_{i}
\end{array}\right) \theta_{1}^{y_{i}}\left(1-\theta_{1}\right)^{n_{i}-y_{i}}\right)^{z_{i}}\left((1-\pi)\left(\begin{array}{l}
n_{i} \\
y_{i}
\end{array}\right) \theta_{2}^{y_{i}}\left(1-\theta_{2}\right)^{n_{i}-y_{i}}\right)^{1-z_{i}}\right]}{\prod_{i=1}^{N}\left[\pi\left(\begin{array}{l}
n_{i} \\
y_{i}
\end{array}\right) \theta_{i}^{y_{i}}\left(1-\theta_{i}\right)^{n_{i}-y_{i}}+(1-\pi)\left(\begin{array}{l}
n_{i} \\
y_{i}
\end{array}\right) \theta_{2}^{y_{i}}\left(1-\theta_{2}\right)^{n_{i}-y_{i}}\right]}
\end{aligned}
$$

onde: $z=\left(z_{1}, \ldots, z_{N}\right), \quad y=\left(y_{1}, \ldots, y_{N}\right)$ e $\boldsymbol{n}=\left(n_{1}, \ldots, n_{N}\right)$

Portanto, a função de verossimilhança ampliada com a introdução das variáveis latentes $\left(Z_{i}, i=1, \ldots, N\right)$ é dada por, 


$$
\begin{aligned}
& L\left(n, \theta_{1}, \theta_{2}, \pi / y, z\right)=L\left(n, \theta_{1}, \theta_{2}, \pi / y\right) \pi\left(n_{2}, y, \theta_{1}, \theta_{2}, \pi / z\right)
\end{aligned}
$$

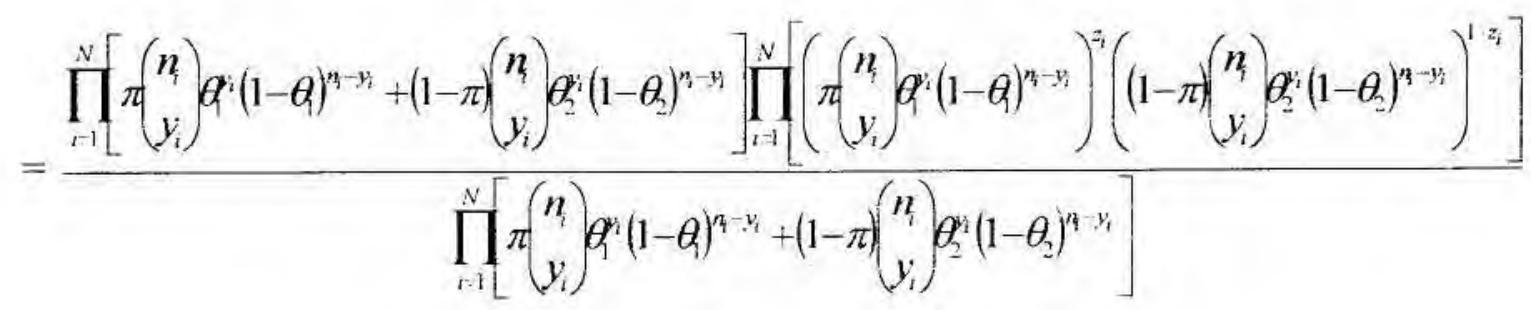

$$
\begin{aligned}
& =\prod_{i=1}^{N}\left[\left(\pi\left(\begin{array}{l}
n_{i} \\
y_{i}
\end{array}\right) \theta_{1}^{y_{i}}\left(1-\theta_{1}\right)^{n_{i}-y_{i}}\right)^{z_{i}}\left((1-\pi)\left(\begin{array}{l}
n_{i} \\
y_{1}
\end{array}\right) \theta_{2}^{y_{i}}\left(1-\theta_{2}\right)^{n_{i}-y_{i}}\right)^{1-z_{i}}\right]
\end{aligned}
$$

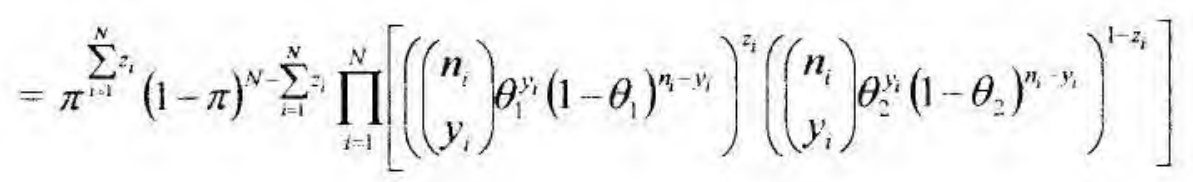

Observar que esta expressão apresenta uma grande simplificação em relação a expressão dada em 4.3 que nos mostra a função de verossimilhança antes de introduzir as variáveis latentes. Este fato irá facilitar o trabalho computacional na análise Bayesiana descrita na próxima seção.

\section{3 - Análise Bayesiana e algoritmo de simulação}

Desenvolvemos uma análise Bayesiana para o modelo de mistura de duas distribuições Binomiais, assumindo as seguintes distribuições a priori para os parâmetros:

(i) $\theta_{1} \sim \operatorname{Beta}\left(a_{1}, b_{1}\right)$

(ii) $\theta_{2} \sim \operatorname{Beta}\left(a_{2}, b_{2}\right)$

(iii) $\pi \sim \operatorname{Beta}(c, d)$ 
Assumindo que as distribuições a priori para os parâmetros $\theta_{1}, \theta_{2}$ e $\pi$ são independentes, a função densidade a priori conjunta para esses parâmetros é dada por,

$$
g\left(\theta_{1}, \theta_{2}, \pi\right) \propto \theta_{1}^{a_{1}-1}\left(1-\theta_{1}\right)^{b_{1}-1} \theta_{2}^{a_{2}-1}\left(1-\theta_{2}\right)^{b_{2}-1} \pi^{c 1}(1-\pi)^{d-1}
$$

Combinando a expressão (4.11) com a função de verossimilhança simplificada por variáveis latentes dada em (4.9), encontramos a densidade a posteriori conjunta para $\theta_{1}, \theta_{2}$ e $\pi$, dada por,

$$
\begin{aligned}
& g\left(\theta_{1}, \theta_{2}, \pi / n, y, z\right) \propto \theta_{1}^{a_{1}-1}\left(1-\theta_{1}\right)^{b_{1}-1} \theta_{2}^{a_{2}-1}\left(1-\theta_{2}\right)^{b_{2}-1} \pi^{c-1}(1-\pi)^{d-1} \pi^{\sum_{i=1}^{N} z_{i}}(1-\pi)^{N-\sum_{i=1}^{N}=} \\
& \prod_{i}^{N}\left[\left(\left(\begin{array}{l}
n_{i} \\
y_{i}
\end{array}\right) \theta_{1}^{y_{i}}\left(1-\theta_{1}\right)^{n_{i}-y_{i}}\right)^{z_{i}}\left(\left(\begin{array}{l}
n_{i} \\
y_{i}
\end{array}\right) \theta_{2}^{y_{i}}\left(1-\theta_{2}\right)^{n_{i}-y_{i}}\right)^{1-z_{i}}\right] \\
& \propto \theta_{1}^{a_{i}+\sum_{i=1}^{N} v_{i}-z_{i}-1}\left(1-\theta_{1}\right)^{a_{1}+\sum_{i=1}^{N}=i\left(n_{i}-y_{i}+1\right.} \theta_{2}^{a_{2}+\sum_{i=1}^{N}\left(1-z_{i}\right) y_{i}-1}\left(1-\theta_{2}\right)^{b_{2}+\sum_{i=1}^{N}\left(1-z_{i}\right)\left(n_{i}-y_{i}\right)-1} \pi^{c+\sum_{i=1}^{N} z_{i}-1}(1-\pi)^{d+N} \sum_{i=1}^{N} z_{i}-1
\end{aligned}
$$

Portanto, as distribuições condicionais necessárias para o uso dos métodos MCMC são dadas por,

(i) $g\left(\theta_{1} / \theta_{2}, \pi, n, z, y\right) \propto \theta_{1}^{a_{1}+\sum_{i=1}^{N} y_{i} z_{i}-1}\left(1-\theta_{1}\right)^{b_{i}}, \sum_{i=1}^{N} z_{i}\left(n_{i} y_{i}\right)-1$

(ii) $g\left(\theta_{2} / \theta_{1}, \pi, n, z, y\right) \propto \theta_{2}^{a_{2}+\sum_{i=1}^{N}\left(1-z_{i} \mid y_{i}-1\right.}\left(1-\theta_{2}\right)^{b_{2}+\sum_{i=1}^{N}\left(1 z_{i}\right) x n_{i} y_{i} \mid-1}$

(iii) $g\left(\pi / \theta_{1}, \theta_{2}, n, z, y\right) \propto \pi^{c+\sum_{i=1}^{N} z_{i}-1}(1-\pi)^{d+N-\sum_{i=1}^{N} z_{i}-1}$

Como as distribuições condicionais para os parâmetros $\theta_{1}, \theta_{2}$ e $\pi$ apresentam formas de distribuições Beta, utilizamos o algoritmo Gibbs Sampling para realizar a simulação dos parâmetros e fazer as inferências desejadas. 
$\mathrm{O}$ algoritmo computacional para gerar amostras dos parâmetros $\theta_{1}, \theta_{2}$ e $\pi$ baseado na introdução de variáveis latentes é dado pelas seguintes etapas:

(i) Dados os valores iniciais $\theta_{1}^{(0)}, \theta_{2}^{(0)}$ e $\pi^{(0))}$, gerar uma amostra $\left(z_{1}, z_{2}, \ldots, z_{N}\right)$ a partir da distribuição de Bernoulli $\mathrm{p} / z_{i}$ com probabilidade de sucesso,

$$
h_{1}=\frac{\pi^{(0)}\left(\begin{array}{l}
n_{i} \\
y_{i}
\end{array}\right)\left(\theta_{1}^{(0)}\right)^{y_{i}}\left(1-\theta_{1}^{(0)}\right)^{n_{i}-y_{i}}}{\pi^{(0)}\left(\begin{array}{l}
n_{1} \\
y_{i}
\end{array}\right)\left(\theta_{1}^{(0)}\right)^{y_{i}}\left(1-\theta_{1}^{(0)}\right)^{n_{i} y_{i}}+\left(1-\pi^{(0)}\right)\left(\begin{array}{l}
n_{i} \\
y_{i}
\end{array}\right)\left(\theta_{2}^{(0)}\right)^{y_{i}}\left(1-\theta_{2}^{(0)}\right)^{n_{i} y_{i}}}, i=1, \ldots, N .
$$

(ii) Gerar $\theta_{i}^{(1)}$ a partir da distribuição Beta $\left(a_{1}+\sum_{i=1}^{N} y_{i} z_{i}, b_{1}+\sum_{i=1}^{N} z_{i}\left(n_{i}-y_{i}\right)\right)$

(iii) Gerar $\theta_{2}^{(1)}$ a partir da distribuição Beta $\left(a_{2}+\sum_{i=1}^{N} y_{i}\left(1-z_{i}\right), b_{2}+\sum_{i=1}^{N}\left(1-z_{i}\right)\left(n_{i}-y_{i}\right)\right)$

(iv) Gerar $\pi^{(1)}$ a partir da distribuição Beta $\left(c+\sum_{i=1}^{N} z_{i}, d+N-\sum_{i=1}^{N} z_{i}\right)$

Repetir (i)-(iv) ate gerar uma amostra de tamanho S. 


\section{Capítulo 5: Discriminação dos modelos}

Nos capitulos 2, 3 e 4 desse trabalho, apresentamos a análise estatística dos modelos Beta-Binomial, Binomial Correlacionado e mistura de duas distribuições Binomiais, que são modelos usados para ajustar a variabilidade extra-Binomial. Freqüentemente, precisamos decidir qual desses modelos se ajusta melhor em relação a determinados conjuntos de dados, e se o ajuste desses modelos é superior ao ajuste do modelo considerando a distribuição Binomial ordinária. Para isto, descrevemos neste capítulo dois métodos usados para discriminar os modelos.

O primeiro método, discutido na seção 5.1 , é o critério de mínima informação (AIC), que é um critério clássico de comparação dos modelos.

O segundo método, de origem Bayesiana, é o fator de Bayes que é a razão das verossimilhanças marginais de dois modelos concorrentes. Discutimos este método em detalhes na seção 5.2, apresentando sua interpretação, alguns estimadores da verossimilhança marginal e comentamos sobre quais desses estimadores iremos considerar em cada modelo. Também nesta seção, utilizamos o fator de Bayes para resolver o teste de hipótese introduzido no capítulo 2 , que compara os modelos Binomial e Beta-Binomial.

\section{1 - Discriminação Clássica dos modelos: AIC}

Uma forma de selecionar modelos na análise Clássica é o uso do critério de mínima informação, conhecido como AIC, proposto por Akaike (ver Box et al., 1994). Esse critério é muito utilizado para discriminar os modelos na área de Séries Temporais, mas com alguns ajustes pode ser utilizado na seleção dos modelos de outras áreas (ver por exemplo, Klein \& Moeschberger, 1997).

Para modelos de Séries Temporais temos,

$$
\mathrm{AIC}=\frac{-2 l(\text { ver } \max .)+2 r}{n}
$$


onde $l$ (ver.max.) denota o logaritmo da função de verossimilhança maximizada, $n$ é o tamanho da série e $r=p+q+1$ denota o número de paràmetros estimados pelo modelo, onde $p$ é a ordem da parte dos modelos auto-regressivos e $q$ é a ordem da parte dos modelos de médias móveis.

Para outras áreas, o critério de mínima informação é dado por,

$$
\mathrm{AIC}=-2 l(\text { ver. } \max .)+2(p)
$$

onde $l$ (ver.max.) denota o logaritmo da função de verossimilhança maximizada e $p$ denota o número de parâmetros estimados pelo modelo.

Os modelos que apresentam menores valores do AIC são os preferidos para ajustar os dados.

É interessante observar que esse critério é extremamente fácil de ser utilizado, pois o "software" SAS nos fornece o logaritmo da função de verossimilhança na análise Clássica dos modelos

\section{2 - Discriminação Bayesiana dos modelos: verossimilhança marginal e}

\section{fator de Bayes.}

A verossimilhança marginal é uma ferramenta de grande importància numa análise Bayesiana quando estamos interessados na construção de testes de hipóteses e na discriminação dos modelos. Para um determinado modelo representado por $M$, a verossimilhança marginal é definida da seguinte da seguinte forma,

$$
p(\underline{r} / M)=\int L\left(\theta_{M}, M\right) \pi\left(\theta_{M} / M\right) \partial \theta_{-M}
$$

onde: $L\left(\theta_{M}, M\right)$ é a função de verossimilhança para o modelo $M$,

$\pi\left(\theta_{M} / M\right)$ é a distribuição a priori conjunta para os parâmetros do modelo $M$,

e $\theta_{-M}$ é o vetor de parâmetros do modelo $M$ 
Quando nosso objetivo é a comparação de dois modelos $M_{1}$ e $M_{2}$, utilizamos o fator de Bayes $B_{12}$, que é definido como a razão das verossimilhanças marginais desses modelos, ou seja:

$$
B_{12}=\frac{P\left(y / M_{1}\right)}{P\left(y / M_{2}\right)}
$$

Desta forma, o fator de Bayes é um valor numérico baseado nos dados, que está a favor de um determinado modelo estatístico e contra um outro modelo. Um interpretação do fator de Bayes sugerido por Kass and Raftery (1995), é dado pela tabela a seguir.

Tabela 5.1: Interpretação do fator de Bayes.

\begin{tabular}{|c|c|c|}
\hline$B_{12}$ & $2 \log B_{12}$ & Evidência a favor de $M_{1}$ \\
\hline$<1$ & $<0$ & Negativa (apoia $M_{2}$ ) \\
\hline $1 \mapsto 3$ & $0 \mapsto 2$ & Vale ser mencionada \\
\hline $3 \mapsto 20$ & $2 \mapsto 6$ & Postiva \\
\hline $20 \mapsto 150$ & $6 \mapsto 10$ & Forte \\
\hline$>150$ & $>10$ & muito forte \\
\hline
\end{tabular}

Pela tabela 5.1. concluimos que quando a verossimilhança marginal do modelo $M_{1}$ for maior que a verossimilhança marginal do modelo $M_{2}$, ou seja quando $B_{12}>1$, o modelo $M_{1}$ apresenta um melhor ajuste dos dados.

Para o caso de $r$ modelos, $M_{1}, M_{2}, \ldots, M_{r}$, escolhemos aquele modelo que apresentar a maior verossimilhança marginal. 


\subsection{1 - Estimação da verossimilhança marginal e aplicação nos modelos}

Em algumas situações elementares envolvendo modelos simples, a verossimilhança marginal dada em (5.3) pode ser calculada analiticamente. Contudo, freqüentemente ela é intratável devido a complexidade no cálculo das integrais, independente de qual distribuição a priori está sendo usada para o modelo em questão. Desta forma, precisamos utilizar algum metodo de aproximação para encontrar um estimador da verossimilhança marginal. Nesta dissertação, consideramos dois métodos de estimação: a aproximação de Monte Carlo e o método de Laplace, descritos respectivamente nas seções 5.2.1.1 e 5.2.1.2. Nestas seções, utilizamos estes métodos para apresentar as expressões dos estimadores das verossimilhanças marginais dos modelos Beta-Binomial, Binomial Correlacionado e mistura de duas distribuições Binomiais, discutidos nos capitulos 2,3 e 4 desta dissertação.

\subsubsection{1 - Aproximação de Monte Carlo}

Como já comentamos nos capitulos anteriores, neste trabalho a análise Bayesiana dos modelos usados para ajustar a variabilidade extra-Binomial é baseada nos métodos de Monte Carlo em Cadeia de Markov (MCMC). Utilizando esses métodos geramos uma amostra $\left(\theta^{(1)}, \theta^{(2)}, \ldots, \theta^{(s)}\right)$ a partir da distribuição a priori $p(\theta)$ e com esta amostra podemos calcular um estimador da verossimilhança marginal (5.3), conhecido como estimador de Monte Carlo, dado por,

$$
\hat{p}(y / M)=\frac{1}{S} \sum_{s=1}^{S} L\left(\theta^{(s)}, M\right)
$$

onde $S$ é o tamanho da amostra obtida por simulação.

Utilizamos a aproximação de Monte Carlo para estimar a verossimilhança marginal em todos os modelos da dissertação, exceto no modelo Binomial onde podemos obter o valor exato da verossimilhança marginal. Na seção 5.2 .2 , onde construímos um teste de hipóteses para comparar os modelos Binomial e Beta-Binomial, apresentamos a verossimilhança marginal exata do modelo Binomial, que sera representado por $M_{1}$. Para os modelos Beta-Binomial, Binomial 
Correlacionado e mistura de duas distribuições Binomiais, representados respectivamente por $M_{2}, M_{3}$ e $M_{4}$, os estimadores de Monte Carlo da verossimilhança marginal são dados por,

1) Modelo Beta-Binomial

$$
p\left(y / M_{2}\right)=\frac{1}{S} \sum_{s=1}^{s}\left\{\prod_{p=1}^{N}\left[\left(\begin{array}{c}
n_{i} \\
y_{i}
\end{array}\right) \frac{\Gamma\left(\alpha^{(s)}+\beta^{(s)}\right)}{\Gamma\left(\alpha^{(s)}\right) \Gamma\left(\beta^{(s)}\right)} \frac{\Gamma\left(\alpha^{(s)}+y_{i}\right) \Gamma\left(n_{i}+\beta^{(s)}-y_{i}\right)}{\Gamma\left(\alpha^{(s)}+\beta^{(s)}+n_{i}\right)}\right]\right\}
$$

2) Modelo Binomial Correlacionado

$$
p\left(y / M_{3}\right)=\frac{1}{S} \sum_{s=1}^{s}\left\{\prod_{i=1}^{N}\left[\left(\begin{array}{l}
n_{i} \\
y_{i}
\end{array}\right)\left(p^{(s)}\right)^{y_{i}}\left(1-p^{(s)}\right)^{n_{n}-y_{i}}\left\{1+\frac{\delta^{(s)}}{2 p^{(s)}\left(1-p^{(s)}\right)}\left[\left(y_{i}-n_{i} p^{(s)}\right)^{2}+y_{i}\left(2 p^{(s)}-1\right)-n_{i}\left(p^{(s)}\right)^{2}\right]\right\}\right]\right\}
$$

3) Modelo de mistura de duas distribuições Binomiais

$$
\hat{p}\left(y / M_{4}\right)=\frac{1}{S} \sum_{s=1}^{S}\left\{\prod_{i=1}^{N}\left[\pi^{(s)}\left(\begin{array}{l}
n_{i} \\
y_{i}
\end{array}\right)\left(\theta_{1}^{(s)}\right)^{y_{i}}\left(1-\theta_{1}^{(s)}\right)^{n_{i}-y_{i}}+\left(1-\pi^{(s)}\right)\left(\begin{array}{l}
n_{i} \\
y_{i}
\end{array}\right)\left(\theta_{2}^{(s)}\right)^{y_{i}}\left(1-\theta_{2}^{(s)}\right)^{n_{i}-y_{i}}\right]\right\}
$$

É importante salientar, que para o modelo Binomial Correlacionado $\left(M_{3}\right)$, como estamos trabalhando com duas estratégias diferentes na análise Bayesiana, vamos encontrar duas estimativas da verossimilhança marginal. Assumindo as distribuições a priori dadas em (3.8), a estimativa de Monte Carlo da verossimilhança marginal será representada por $\hat{p}\left(y / M_{31}\right)$, ao passo que assumindo as distribuições a priori dadas em (3.12), a estimativa de Monte Carlo da verossimilhança marginal será representada por $\hat{p}\left(y / M_{32}\right)$. 


\subsubsection{2 - Método de Laplace}

A aproximação de Laplace (ver Tierney \& Kadane, 1986) é uma técnica numérica muito utilizada na inferência Bayesiana para resolver integrais. Este método de aproximação pode ser utilizando satisfazendo certas condições de regularidade e a aproximação é bem razoável para tamanhos amostrais grandes.

Representamos por $I$ a verossimilhança marginal dada em (5.3). A aproximação de Laplace para $I$ é dada por,

$$
\hat{I}=(2 \pi)^{d / 2} \sigma L(\hat{\theta}) \pi(\hat{\theta})
$$

onde: d é a dimensão do vetor de parâmetros $\theta$.

$$
\begin{aligned}
& \hat{\sigma}=\left\{-\partial^{2} \log [L(\hat{\theta}) \pi(\hat{\theta})]\right\}^{-1 / 2} \\
& \text { e } \hat{\theta} \text { é o vetor de estimadores de máxima verossimilhança de } \theta .
\end{aligned}
$$

Como o nosso objetivo na análise Bayesiana dos modelos é voltado para o uso dos métodos MCMC, onde a discriminação dos modelos é baseada nos estimadores de Monte Carlo, não utilizamos o método de Laplace para estimar a verossimilhança marginal de todos os modelos discutidos até aqui. Escolhemos apenas o modelo Beta-Binomial para encontrar uma estimativa de Lapalce da verossimilhança marginal e comparamos este valor com a estimativa de Monte Carlo do modelo. Utilizando a expressão (5.9), o estimador de Laplace da verossimilhança marginal para o modelo Beta-Binomial é dado por, 


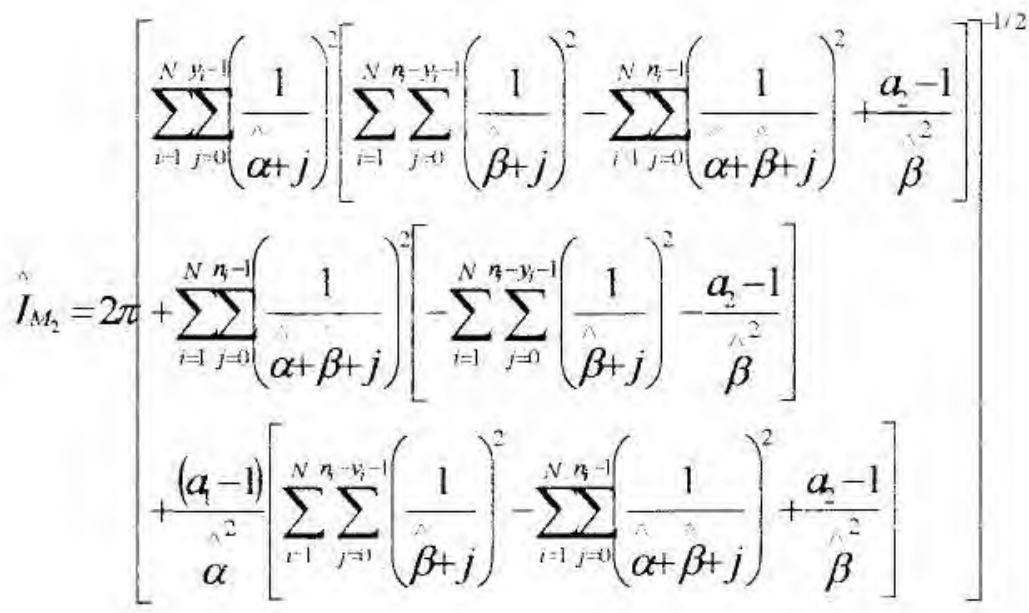

$$
\begin{aligned}
& \prod_{t=1}^{N}\left[\left(\begin{array}{l}
n_{i} \\
y_{i}
\end{array}\right) \frac{\prod_{j=0}^{y_{1}}(\alpha+j)^{n_{1}-y_{1} i}\left(\prod_{j=0}^{i}(\beta+j)\right.}{\prod_{j=0}^{n_{1}-1}(\alpha+\beta+j)}\right] \frac{b_{1}^{\alpha_{1}}}{\Gamma\left(a_{1}\right)} \alpha^{h_{1} t} e^{-b_{1} \alpha} \frac{b_{2}^{a_{2}}}{\Gamma\left(a_{2}\right)} \beta^{a_{2}+1} e^{-b_{2} \beta}
\end{aligned}
$$

5.2.2 - Aplicação do Fator de Bayes no teste de hipóteses envolvendo os modelos Binomial e Beta-Binomial.

Como já comentamos no capitulo 2 , quando utilizamos a reparametrização $(\xi, \omega)$ dada em (2.6), o modelo Beta-Binomial tende suavemente para o modelo Binomial quando o parâmetro $\omega \rightarrow 0$. Este fato permite a construção do teste de hipótese dado em (2.8), que compara os modelos Binomial e Beta-Binomial através do parâmetro $\omega$.

Para resolver este teste de hipótese, podemos utilizar o fator de Bayes, que neste caso é dado por,

$$
B(\omega)=\frac{P\left(y / H_{0}\right)}{P\left(y / H_{1}\right)}=\frac{\int_{0}^{1} L\left(p, M_{1}\right) \pi\left(p / M_{1}\right) d p}{\int_{0}^{\infty} \int_{0}^{1} L\left(\xi, \omega, M_{2}\right) \pi\left(\xi, \omega / M_{2}\right) d \xi d \omega}
$$

onde $\pi\left(p / M_{1}\right)$ e $\pi\left(\xi, \omega / M_{2}\right)$ são as distribuições a priori associadas ao modelos $\operatorname{Binomial}\left(M_{1}\right)$ e $\operatorname{Beta-Binomial}\left(M_{2}\right)$ e $L\left(p, M_{1}\right)$ e $L\left(\xi, \omega, M_{2}\right)$ são as funções de verossimilhança desses modelos. 
Para calcular a verossimilhança marginal do numerador do fator de Bayes, que está associada a hipótese $H_{4}$ favoráel ao modelo Binomial, assumimos uma distribuição a priori Uniforme $(0,1)$ para o parâmetro $p$. Desta forma, podemos determinar o valor exato de $P\left(y / H_{0}\right)$ pelo uso da função Beta, ou seja,

$$
P\left(y / H_{0}\right)=P\left(y / M_{1}\right)=\int_{i=1}^{1} \prod_{i=1}^{N}\left[\left(\begin{array}{l}
n_{i} \\
y_{i}
\end{array}\right) p^{y_{i}}(1-p)^{n_{1}-y_{i}}\right] d p=\frac{\Gamma\left(\sum_{i=1}^{N} y_{i}+1\right) \Gamma\left(\sum_{i=1}^{N}\left(n_{i}-y_{i}\right)+1\right)}{\Gamma\left(\sum_{i=1}^{N} n_{i}+2\right)}
$$

$$
\text { onde } k=\left[\prod_{i=1}^{N}\left(\begin{array}{l}
n_{i} \\
y_{i}
\end{array}\right)\right] \text {. }
$$

Com relação ao denominador $P\left(y / H_{1}\right)$ não podemos determinar o valor exato e devemos utilizar algum método de aproximação para estimar a verossimilhança marginal. $\mathrm{O}$ método escolhido foi o método de Laplace descrito na seção 5.2.1.2. Para o parâmetro $\xi$, assumimos a mesma distribuição a priori Uniforme $(0,1)$ que assumimos para o parâmetro $p$ do modelo Binomial. Com relação ao parâmetro $\omega$, não vamos especificar nenhuma distribuição a priori e vamos calcular o fator de Bayes considerando diferentes valores de $\omega$.

O estimador de Laplace para $P\left(y / H_{1}\right)$ é dado por,

$$
I_{H_{1}}=(2 \pi)^{1 / 2}\left[\sum_{i=1}^{N} \sum_{j=0}^{y_{i}}\left(\frac{1}{\xi+\omega j}\right)^{2}+\sum_{i=1}^{N} \sum_{j=0}^{n_{i}} \sum_{j}^{y_{i}}\left(\frac{1}{1-\hat{\xi}+\omega j}\right)^{2}\right]^{-1 / 2}\left[\prod_{i=1}^{N}\left[\left(\begin{array}{l}
n_{i} \\
y_{i}
\end{array}\right) \frac{\prod_{j=0}^{y_{j}-1}(\xi+\omega j)^{n_{i}-y_{i}-1}(1-\hat{\xi}+\omega j)}{\prod_{j=0}^{n_{i}-1}(1+\omega j)}\right]\right.
$$

Portanto, para vários valores de $\omega$, encontramos $\hat{I}_{H_{\mathrm{H}}}$ e calculamos cstimativas do fator de Bayes. A interpretação do fator de Bayes e a conclusão do teste de hipóteses são feitos com base na tabela 5.1 , que apresentamos no início da seção 5.2 . 


\section{Capítulo 6: Variabilidade extra-Binomial}

\section{na presença de covariáveis}

Nos capitulos anteriores da dissertação, o nosso estudo esteve voltado para os modelos usados para ajustar dados de contagens de sucessos, que apresentam uma variabilidade superior ou inferior a variabilidade do modelo Binomial. Neste capitulo, o nosso interesse continua sendo a análise de modelos para dados de contagens de sucessos, mas considerando a presença de covariaveis, ou seja analisamos situações onde temos variáveis explanatórias associadas às observações.

Em muitas aplicações encontramos conjuntos de dados dessa forma. Por exemplo, em estudos toxicológicos, a unidade experimental pode ser ninhadas de animais e a variável resposta é o número de animais com alguma anormalidade. A incidência dessa anormalidade pode variar entre as ninhadas devido a influências genéticas ou ambientais e o uso de covariáveis torna-se necessário. Um modelo que é muito utilizado para ajustar dados com essas caracteristicas é o modelo de regressão logística, discutido na seção 6.1. Contudo, em algumas situações, quando todas as variáveis explanatórias do modelo forem ajustadas, a variabilidade observada dos dados pode ser maior do que a variabilidade esperada assumindo o modelo de regressão logística. Neste caso, para melhorar o ajuste, temos duas possibilidades: adicionar outras variáveis explanatórias no modelo ou introduzir no modelo uma fonte da variabilidade extra-Binomial. Freqüentemente, a segunda opção tem sido escolhida (ver Williams, 1982) e na seção 6.2 apresentamos dois modelos usados para ajustar a variabilidade extra-Binomial na presença de covariáveis. Na seção 6.3, apresentamos a análise estatística dos modelos discutidos nas seções 6.1 e 6.2 e comentamos sobre a discriminação dos modelos na presença de covariáveis.

\section{1 - Modelo de regressão logística}

Em determinados experimentos, utilizamos as técnicas de análise de regressão quando estamos interessados em investigar como as mudanças ocorridas em uma ou mais variáveis 
explicativas, também chamadas de variáveis independentes ou covariáveis, afetam uma outra variável, denominada variável resposta ou dependente. Essas técnicas permitem verificar se existe realmente uma relação significativa entre essas variáveis, qual o tipo de relação, além de obter a predição das variáveis respostas a partir das variáveis explicativas.

O exemplo mais comum de modelagem na análise de regressão é o modelo de regressão linear. Consideramos que $m$ covariáveis, $W_{i 1}, W_{i 2}, \ldots, W_{i m}$, estão associadas a uma variável resposta $V_{1}$. No modelo de regressão linear podemos expressar o valor médio da variável resposta como uma equação linear das variáveis explicativas, ou seja,

$$
E\left(V_{i} / W_{i 1}, \ldots, W_{i m}\right)-\sum_{j=0}^{m} \beta_{j} W_{i j}
$$

onde: $w_{i 0}=1, i=1, \ldots, N$ e $E\left(V_{1} / W_{i 1}, \ldots, W_{i m}\right)$ é contínua e pode assumir qualquer valor entre $-\infty$ e $\infty$.

No caso da variável resposta ser binária ou dicotômica, utilizamos o modelo de regressão logística, que surgiu da necessidade de modelos mais satisfatórios para dados qualitativos e pela inadequação ao aplicar regressão linear para variáveis dependentes qualitativas. Considerando como binária a variável resposta $V_{i}$ e representando por $p_{1}$ a média da variável resposta $E\left(V_{1} / W_{i 1}, \ldots, W_{i m}\right)$, o modelo de regressão logística é definido por,

$$
p_{i}=\frac{e^{\sum_{j=0}^{m} \beta_{j} W_{i j}}}{1+e^{\sum_{j=1}^{m} \beta_{j} W_{i j}}}
$$

Uma transformação de $p_{i}$, denominada transformação logito, é dada por,

$$
\lambda_{i}=\log \left(\frac{p_{i}}{1-p_{i}}\right)=\sum_{i=1}^{m} \beta_{j} W_{i}
$$

A importância dessa transformação é que $\lambda_{i}$ tem muitas das propriedades desejáveis de um modelo de regressão linear. $\mathrm{O}$ logito $\lambda_{i}$, é linear em seus parâmetros, pode ser contínuo e pode estar entre $-\infty$ e $\infty$, dependendo do limite das variáveis explicativas. 
Nesse trabalho, como nosso interesse é voltado para o número de sucessos em $n_{i}$ ensaios, consideramos que a i-ésima resposta, $(1 \leq i \leq N)$, é uma contagem de $y_{i}$ sucessos e $n_{i}-y_{i}$ fracassos. Associado a cada resposta, existem os valores de $m$ covariáveis $W_{i 1}, W_{i 2}, \ldots, W_{i m}$, onde a matriz $W(\mathrm{Nxm})$ é de posto $m$. Neste caso, assumindo o modelo de regressão logística temos,

$$
Y_{i} \sim \operatorname{Binomial}\left(n_{i}, p_{i}\right)
$$

onde $p_{i}$ é dado em (6.2).

Como já discutimos anteriormente, quando temos covariáveis associadas às observações, o modelo de regressão logística pode não ser o melhor para ajustar os dados devido a presença da variabilidade extra-Binomial. Para resolver este problema, apresentamos na seção 6.2 dois modelos alternativos que englobam no modelo de regressão logistica uma fonte da variabilidade extra-Binomial.

\section{2 - Modelos para ajustar a variabilidade extra-Binomial na presença de covariáveis}

Para modelar a variabilidade extra-Binomial na presença de covariáveis, assumimos para a variável aleatória $Y_{i}$, que representa o número de sucessos em $n_{t}$ ensaios, o modelo Binomial Correlacionado dado pela expressão (3.2) e discutido no capítulo 3. Este modelo como já comentamos, assume que a fonte da variabilidade extra-Binomial é a correlação entre as variáveis binárias $U_{i 1}, U_{i 2}, \ldots ., U_{i n_{i}}$ de $Y_{i}=\sum_{j=1}^{n_{i}} U_{i j}$. A diferença é que no capítulo 3 , analisamos esse modelo sem a presença de covariáveis e assim, assumimos uma mesma probabilidade de sucesso $p$ para todos os ensaios do experimento. Quando temos a presença das covariáveis $W_{i 1}, W_{i 2}, \ldots, W_{i m}, \quad i=1, \ldots, N$, associadas as contagens de sucessos $y_{1}, y_{2}, \ldots, y_{N}$, as probabilidades de sucesso e de fracasso dos ensaios referentes a essas contagens de sucessos, podem ser diferentes, pois elas dependem dos valores das covariáveis. Assim, o modelo Binomial Correlacionado para $Y_{i}, i=1, \ldots, N$, na presença de covariáveis é dado por, 


$$
\begin{aligned}
& f\left(y_{i} / n_{i}, \delta, \beta, W_{i}\right)=\left(\begin{array}{c}
n_{i} \\
y_{i}
\end{array}\right) p_{i}^{y_{i}}\left(1-p_{i}\right)^{n_{i}-y_{i}} A_{i}\left(y_{i}, n_{i}, \delta, \beta, W_{i}\right) \\
& \text { onde: } A_{i}\left(y_{i}, n_{i}, \delta, \beta, W_{i}\right)=1+\frac{\delta}{2 p_{i}\left(1-p_{i}\right)}\left[\left(y_{i}-n_{i} p_{i}\right)^{2}+y_{i}\left(2 p_{i}-1\right)-n_{i} p_{i}^{2}\right], \\
& p_{i} \text { é dado em }(6.2), \beta^{\prime}=\left(\beta_{0}, \beta_{1}, \ldots, \beta_{m}\right) \text { e } W_{i}=\left(W_{i 1}, \ldots, W_{i m}\right)
\end{aligned}
$$

Observamos que neste modelo, a correlação intra-classe $\delta$ entre as variáveis binárias $U_{i 1}, U_{12}, \ldots, U_{i n_{i}}$ de $Y_{i}$, ao contrário das probabilidades de sucesso $p_{1}, p_{2}, \ldots, p_{N}$, é homogênea e independente dos valores das covariáveis $W_{i 1}, W_{i 2}, \ldots, W_{i m}, i=1, \ldots, N$.

Uma outra possibilidade de modelagem é considerar correlações intra-classes diferentes para cada variável $Y_{i}$, com covariáveis $W_{i 1}, W_{i 2}, \ldots, W_{i m}, i=1, \ldots, N$. Neste caso, a função densidade de $Y_{i}, i=1, \ldots, N$, é dada por,

$$
\begin{aligned}
& f\left(y_{i} / n_{i}, \delta_{i}, \beta, W_{i}\right)=\left(\begin{array}{c}
n_{i} \\
y_{i}
\end{array}\right) p_{i}^{y_{i}}\left(1-p_{i}\right)^{n_{i}-y_{i}} A_{i}\left(y_{i}, n_{i}, \delta_{i}, \beta, W_{i}\right) \\
& \text { onde: } A_{i}\left(y_{i}, n_{i}, \delta_{i}, \beta, W_{i}\right)=1+\frac{\delta_{i}}{2 p_{i}\left(1-p_{i}\right)}\left[\left(y_{i}-n_{i} p_{i}\right)^{2}+y_{i}\left(2 p_{i}-1\right)-n_{i} p_{i}^{2}\right]
\end{aligned}
$$

Desta forma, consideramos esses dois modelos, o Binomial Correlacionado com correlações homogêneas e o Binomial Correlacionado com correlações diferentes, para ajustar a variabilidade extra-Binomial na presença de covariáveis. Na análise de dados reais, descrita no capitulo 7, comparamos o ajuste desses modelos com o ajuste do modelo de regressão logística, para verificar se a variabilidade extra-Binomial está presente nos dados.

\section{3 - Análise estatística dos modelos}

Nesta seção, analisamos os modelos discutidos nas seções 6.1 e 6.2 , utilizando os métodos Clássicos e Bayesianos, seguindo os mesmos procedimentos dos capítulos anteriores quando não utilizamos covariáveis. Na seção 6.3.1, apresentamos a análise Clássica dos modelos 
de regressão logistica e Binomial Correlacionado com correlações homogêneas na presença de covariáveis. Omitimos a analise Clássica do modelo Binomial Correlacionado com correlações diferentes, devido ao grande número de parâmetros presentes neste modelo, que acabam dificultando a obtenção dos EMV pelo método de Newton Raphson. Na seção 6.3.2, apresentamos a análise Bayesiana dos três modelos usados para ajustar os dados na presença de covariáveis.

\subsection{1 - Análise Clássica dos modelos}

Considerando independentes as contagens de sucessos $y_{1}, y_{2}, \ldots, y_{N}$, a função de verossimilhança para o modelo de regressão logistica, dado em (6.4), é dada por,

$$
\begin{aligned}
& L\left(\beta, W_{i}\right)=\prod_{i=1}^{N}\left[\left(\begin{array}{l}
n_{i} \\
y_{i}
\end{array}\right)\left(\frac{e^{\sum_{j=0}^{m} \beta_{j} W_{i j}}}{1+e^{\sum_{j=0}^{m} \beta_{i} \boldsymbol{w}_{i j}}}\right)^{v_{i}}\left(\frac{1}{1+e^{\sum_{j=0}^{m} \beta_{j} \boldsymbol{w}_{i j}}}\right)^{n_{i}-y_{i}}\right] \\
& =\prod_{i=1}^{N}\left[\left(\begin{array}{l}
n_{i} \\
y_{i}
\end{array}\right) \frac{e^{y_{i} \sum_{j=0}^{n} \beta W_{i}}}{\left(1+e^{y_{i} \sum_{i=0}^{n} \beta_{i}}\right)^{n_{3}}}\right]
\end{aligned}
$$

onde: $\beta^{\prime}=\left(\beta_{0}, \beta_{1}, \ldots, \beta_{m}\right)$ e $W_{i}=\left(W_{t 1}, \ldots, W_{\iota m}\right)$

Aplicando o logaritmo na expressão (6.7) temos:

$$
\begin{aligned}
& l\left(\beta, W_{i}\right)=\log \left[L\left(\beta, W_{i}\right)\right]= \\
& =\log \left\{\prod_{i=1}^{N}\left[\left(\begin{array}{l}
n_{i} \\
y_{i}
\end{array}\right) \frac{e^{\sum_{i=0}^{n} \beta_{W_{i}}}}{\left(1+e^{\cdot \sum_{i}^{n} \beta W}\right)^{n_{3}}}\right]\right\}=\sum_{i=1}^{N}\left\{\log \left[\left(\begin{array}{l}
n_{i} \\
y_{i}
\end{array}\right) \frac{e^{y_{i} \sum_{j=0}^{m} \beta_{i} W_{i j}}}{\left(1+e^{\sum_{i} \sum_{0}^{m} \beta_{i} W_{i}}\right)^{n_{i}}}\right]\right\}
\end{aligned}
$$


$=\sum_{i=1}^{N} \log \left(\begin{array}{l}n_{i} \\ y_{i}\end{array}\right)+\beta_{0} \sum_{i=1}^{N} y_{i}+\sum_{i=1}^{N}\left[y_{i} \sum_{j=1}^{m} \beta_{j} W_{i}\right]-\sum_{i=1}^{N}\left[n_{i} \log \left(1+u_{i}\right)\right]$

onde: $u_{i}=e^{\beta_{2}+\sum_{j=1}^{m} \beta_{j} W_{i:}}$

Derivando o logaritmo da função de verossimilhança em relação aos parâmetros $\beta_{0}$ e $\beta_{j}, j=1, \ldots, m$, temos:

$$
\begin{aligned}
& \frac{\partial\left(\beta, W_{i}\right)}{\partial \beta_{0}}=\sum_{i=1}^{N} y_{i}-\sum_{i=1}^{N}\left[n_{i}\left(\frac{u_{i}}{1+u_{i}}\right)\right] \\
& \frac{\partial\left(\beta, W_{i}\right)}{\partial \beta_{j}}=\sum_{i=1}^{N} y_{i} W_{i j}-\sum_{i=1}^{N}\left[n_{i} W_{i j}\left(\frac{u_{i}}{1+u_{i}}\right)\right]
\end{aligned}
$$

Igualando estas expressões a 0 , observamos que as equações não são lineares em relação aos parâmetros $\beta_{0}, \beta_{1}, \ldots, \beta_{m}$. Assim, utilizamos o método iterativo de Newton Raphson descrito no capitulo 2, para encontrar os EMV desses parâmetros.

As derivadas de segunda ordem do logaritmo da função de verossimilhança em relação a $\beta_{0}$ e $\beta_{j}, j=1, \ldots, m$, necessárias para o método de Newton-Raphson são dadas por,

$$
\begin{aligned}
& \frac{\partial^{2} l\left(\beta, W_{i}\right)}{\partial \beta_{0}^{2}}=-\sum_{i=1}^{N}\left[n_{i}\left(\frac{u_{i}\left(1+u_{i}\right)-u_{i}^{2}}{\left(1+u_{i}\right)^{2}}\right)\right] \\
& \frac{\partial^{2} l\left(\beta_{\sim}, W_{i}\right)}{\partial \beta_{j}^{2}}=-\sum_{i=1}^{N}\left[n_{i} W_{i j}^{2}\left(\frac{u_{i}\left(1+u_{i}\right)-u_{i}^{2}}{\left(1+u_{i}\right)^{2}}\right)\right] \\
& \frac{\partial^{2} l\left(\beta, W_{i}\right)}{\partial \beta_{i} \partial \beta_{j}}=-\sum_{i=1}^{N}\left[n_{i} W_{i j}\left(\frac{u_{i}\left(1+u_{i}\right)-u_{i}^{2}}{\left(1+u_{i}\right)^{2}}\right)\right]
\end{aligned}
$$




$$
\frac{\partial^{2} f\left(\beta, W_{i}\right)}{\partial \beta_{j} \partial \beta_{k}}=-\sum_{i=1}^{N}\left[n_{i} W_{i j} W_{t k}\left(\frac{u_{i}\left(1+u_{i}\right)-u_{i}^{2}}{\left(1+u_{i}\right)^{2}}\right)\right] \text { onde } j \neq k
$$

Assumindo o modelo Binomial Correlacionado com correlações homogêneas na presença de covariáveis, dado em (6.5), e considerando independentes as contagens de sucessos $y_{1}, y_{2}, \ldots, y_{N}$, temos,

$$
\begin{aligned}
& L\left(\delta, \beta, W_{i}\right)=\prod_{i=1}^{N}\left[\left(\begin{array}{l}
n_{i} \\
y_{i}
\end{array}\right)\left(\frac{e^{\sum_{j=0}^{m} \beta_{j} W_{i j}}}{1+e^{\sum_{j=0}^{m} \beta_{j} \boldsymbol{w}_{i j}}}\right)^{y_{i}}\left(\frac{1}{1+e^{\sum_{j=0}^{m} \beta_{j} \boldsymbol{w}_{i j}}}\right)^{n_{i}-y_{i}} A_{i}\left(n_{i}, y_{i}, \delta, \beta, W_{i}\right)\right] \\
& =\prod_{i=1}^{N}\left[\left(\begin{array}{l}
n_{i} \\
y_{i}
\end{array}\right) \frac{e^{y_{i} \sum_{j=0}^{\pi} \beta B_{W_{i}}}}{\left(1+e^{y_{i} \sum_{j=0}^{n} \beta W_{i}}\right)^{n_{i}}} A_{i}\left(n_{i}, y_{i}, \delta, \beta, W_{i}\right)\right]
\end{aligned}
$$

onde: $\underset{\sim}{\beta}$ e $W_{\sim}$ são dados em (6.7) e $A_{i}\left(n_{i}, y_{i}, \delta, \beta, W_{i}\right)$ é dado em (6.5)

Aplicando o logaritmo na expressão (6.11) e desenvolvendo de maneira similar à expressão (6.8), temos,

$$
\begin{gathered}
l\left(\delta, \beta, W_{i}\right)=\log \left[L\left(\delta, \beta, W_{i}\right)\right]= \\
=\sum_{i=1}^{N} \log \left(\begin{array}{l}
n_{i} \\
y_{i}
\end{array}\right)+\beta_{0} \sum_{i=1}^{N} y_{i}+\sum_{i=1}^{N}\left[y_{i} \sum_{j=1}^{m} \beta_{j} W_{i j}\right]-\sum_{i=1}^{N}\left[n_{i} \log \left(1+u_{i}\right)\right]+\sum_{i=1}^{N}\left\{\log \left[A_{i}\left(n_{i}, y_{i}, \delta, \beta, W_{i}\right)\right]\right\}
\end{gathered}
$$

onde: $u_{i}$ é dado em (6.8) 


$$
\begin{aligned}
& \mathrm{e} \sum_{i=1}^{N}\left\{\log \left[A_{i}\left(n_{i}, y_{i}, \delta, \beta, W_{i}\right)\right]\right\}= \\
& =\sum_{i=1}^{N} \log \left\{1+\frac{\delta\left(1+u_{i}\right)}{2 u_{i}}\left[\left(y_{i}-n_{i} \frac{u_{i}}{1+u_{i}}\right)^{2}+y_{i}\left(2 \frac{u_{i}}{1+u_{i}}-1\right)-n_{i}\left(\frac{u_{i}}{1+u_{i}}\right)^{2}\right]\right\} \\
& =\sum_{i=1}^{N} \log \left\{1+\frac{\delta y_{i}\left(y_{i}-1\right)}{2}\left[\frac{\left(1+u_{i}\right)^{2}}{u_{i}}\right]+\delta y_{i}\left(1-n_{i}\right)\left(1+u_{i}\right)+u_{i} \frac{\delta n_{i}\left(n_{i}-1\right)}{2}\right\}
\end{aligned}
$$

As derivadas de primeira ordem do logaritmo da função de verossimilhança em relação aos parâmetros $\delta, \beta_{0}$ e $\beta_{j}, j=1, \ldots, m$, são dadas por,

$$
\begin{aligned}
& \frac{\partial l\left(\delta, \beta, W_{i}\right)}{\partial \delta}=\sum_{i=1}^{N}\left\{\frac{\left(y_{i}-n_{i} p_{i}\right)^{2}+y_{i}\left(2 p_{i}-1\right)-n_{i} p_{i}^{2}}{2 p_{i}\left(1-p_{i}\right)+\delta\left[\left(y_{i}-n_{i} p_{i}\right)^{2}+y_{i}\left(2 p_{i}-1\right)-n_{i} p_{i}^{2}\right]}\right\} \\
& \frac{\partial l\left(\delta, \beta, W_{i}\right)}{\partial \beta_{0}}=\sum_{i=1}^{N} y_{i}-\sum_{i=1}^{N}\left[n_{i}\left(\frac{u_{i}}{1+u_{i}}\right)\right] \\
& +\sum_{i=1}^{N}\left\{\frac{\frac{\delta y_{i}\left(y_{i}-1\right)}{2}\left[2\left(1+u_{i}\right)-\frac{\left(1+u_{i}\right)^{2}}{u_{i}}\right]+u_{i}\left[\delta y_{i}\left(1-n_{i}\right)+\frac{\delta n_{i}\left(n_{i}-1\right)}{2}\right]}{1+\frac{\delta y_{i}\left(y_{i}-1\right)}{2}\left[\frac{\left(1+u_{i}\right)^{2}}{u_{i}}\right]+\delta y_{i}\left(1-n_{i}\right)\left(1+u_{i}\right)+u_{i} \frac{\delta n_{i}\left(n_{i}-1\right)}{2}}\right\} \\
& \frac{\partial l\left(\delta, \beta, W_{i}\right)}{\partial \beta_{j}}=\sum_{i=1}^{N} y_{i} W_{i j}-\sum_{i=1}^{N}\left[n_{i} W_{i j}\left(\frac{u_{i}}{1+u_{i}}\right)\right] \\
& +\sum_{i=1}^{N}\left\{\begin{array}{c}
\frac{\delta y_{i}\left(y_{i}-1\right)}{2}\left[2\left(1+u_{i j} \frac{\left(1+u_{i}\right)^{2}}{u_{i}}\right]+u_{i}\left[\delta y_{i}\left(1-n_{i}\right)+\frac{\delta n_{i}\left(n_{i}-1\right)}{2}\right]\right. \\
1+\frac{\delta y_{i}\left(y_{i}-1\right)}{2}\left[\frac{\left(1+u_{i}\right)^{2}}{u_{i}}\right]+\delta y_{i}\left(1-n_{i}\right)\left(1+u_{i}\right)+u_{i} \frac{\delta n_{i}\left(n_{i}-1\right)}{2}
\end{array}\right\}
\end{aligned}
$$


Como as expressões dadas em (6.13) não são lineares em relação aos parâmetros, utilizamos também neste modelo o metodo iterativo de Newton Raphson para encontrar os EMV de $\delta, \beta_{0}$ e $\beta_{j}, j=1, \ldots, m$.

Neste modelo, as derivadas de segunda ordem são dadas por,

$$
\begin{aligned}
& \left.\frac{\partial^{2} l\left(\delta, \beta, W_{i}\right)}{\partial \delta^{2}}=-\sum_{i=1}^{N}\left\{\left[\frac{\left(y_{i}-n_{i} p_{i}\right)^{2}+y_{i}\left(2 p_{i}-1\right)-n_{i} p_{i}^{2}}{2 p_{i}\left(1-p_{i}\right)+\delta\left[\left(y_{i}-n_{i} p_{i}\right)^{2}+y_{i}\left(2 p_{i}-1\right)-n_{i} p_{i}^{2}\right.}\right]\right]^{2}\right\} \\
& \frac{\partial^{2} l\left(\delta, \beta, W_{i}\right)}{\partial \beta_{0}^{2}}=-\sum_{i=1}^{N}\left[n_{i}\left(\frac{\boldsymbol{u}_{i}\left(1+\boldsymbol{u}_{i}\right)-\boldsymbol{u}_{i}^{2}}{\left(1+\boldsymbol{u}_{i}\right)^{2}}\right)\right] \\
& +\sum_{i=1}^{N}\left\{\frac{\frac{\delta y_{i}\left(y_{i}-1\right)}{2}\left[-2+\frac{\left(1+u_{i}\right)^{2}}{u_{i}}\right]+u_{i}\left[\delta y_{i}\left(1-n_{i}\right)+\frac{\delta n_{i}\left(n_{i}-1\right)}{2}\right]}{1+\frac{\delta y_{i}\left(y_{i}-1\right)}{2}\left[\frac{\left(1+u_{i}\right)^{2}}{u_{i}}\right]+\delta y_{i}\left(1-n_{i}\right)\left(1+u_{i}\right)+u_{i} \frac{\delta n_{i}\left(n_{i}-1\right)}{2}}\right\} \\
& -\sum_{i=1}^{N}\left\{\left[\frac{\frac{\delta y_{i}\left(y_{i}-1\right)}{2}\left[2\left(1+u_{i}\right)-\frac{\left(1+u_{i}\right)^{2}}{u_{i}}\right]+u_{i}\left[\delta y_{i}\left(1-n_{i}\right)+\frac{\delta n_{i}\left(n_{i}-1\right)}{2}\right]}{1+\frac{\delta y_{i}\left(y_{i}-1\right)}{2}\left[\frac{\left(1+u_{i}\right)^{2}}{u_{i}}\right]+\delta y_{i}\left(1-n_{i}\right)\left(1+u_{i}\right)+u_{i} \frac{\delta n_{i}\left(n_{i}-1\right)}{2}}\right]^{2}\right\}
\end{aligned}
$$

$$
\begin{aligned}
& \frac{\partial^{2}\left(\delta, \beta, W_{i}\right)}{\partial \beta_{j}^{2}}=-\sum_{i=1}^{N}\left[n_{i} W_{i j}^{2}\left(\frac{u_{i}\left(1+u_{i}\right)-u_{i}^{2}}{\left(1+u_{i}\right)^{2}}\right)\right] \\
& +\sum_{i=1}^{N}\left\{W_{i j}^{2} \frac{\frac{\delta y_{i}\left(y_{i}-1\right)}{2}\left[-2+\frac{\left(1+u_{i}\right)^{2}}{u_{i}}\right]+u_{i}\left[\delta y_{i}\left(1-n_{i}\right)+\frac{\delta n_{i}\left(n_{i}-1\right)}{2}\right]}{\left.\left.1+\frac{\delta y_{i}\left(y_{i}-1\right)\left[\left(1+u_{i}\right)^{2}\right.}{u_{i}}\right]+\delta y_{i}\left(1-n_{i}\right)\left(1+u_{i}\right)+u_{i} \frac{\delta n_{i}\left(n_{i}-1\right)}{2}\right\}}\right\} \\
& \left.-\sum_{i=1}^{N}\left\{W_{i j}^{2}\left[\frac{\frac{\delta y_{i}\left(y_{i}-1\right)}{2}\left[2\left(1+u_{i}\right)-\frac{\left(1+u_{i}\right)^{2}}{u_{i}}\right]+u_{i}\left[\delta y_{i}\left(1-n_{i}\right)+\frac{\delta n_{i}\left(n_{i}-1\right)}{2}\right]}{1+\frac{\delta y_{i}\left(y_{i}-1\right)}{2}\left[\frac{\left(1+u_{i}\right)^{2}}{u_{i}}\right]+\delta y_{i}\left(1-n_{i}\right)\left(1+u_{i}\right)+u_{i} \frac{\delta n_{i}\left(n_{i}-1\right)}{2}}\right]\right]^{2}\right\}
\end{aligned}
$$




$$
\begin{aligned}
& \frac{\partial^{2} l\left(\delta, \beta, W_{i}\right)}{\partial \beta_{0} \partial \beta_{j}}=\frac{\partial^{2} l\left(\delta, \beta, W_{i}\right)}{\partial \beta_{j} \partial \beta_{0}} \\
& =-\sum_{i=1}^{N}\left[n_{i} W_{i j}\left(\frac{u_{i}\left(1+u_{i}\right)-u_{i}^{2}}{\left(1+u_{i}\right)^{2}}\right)\right] \\
& +\sum_{i=1}^{v}\left\{W_{y j} \frac{\frac{\delta y_{i}\left(y_{i}-1\right)}{2}\left[-2+\frac{\left(1+u_{i}\right)^{2}}{u_{i}}\right]+u_{i}\left[\delta y_{i}\left(1-n_{i}\right)+\frac{\delta n_{i}\left(n_{i}-1\right)}{2}\right]}{1+\frac{\delta y_{i}\left(y_{i}-1\right)}{2}\left[\frac{\left(1+u_{i}\right)^{2}}{u_{i}}\right]+\delta y_{i}\left(1-n_{i}\right)\left(1+u_{i}\right)+u_{i} \frac{\delta n_{i}\left(n_{t}-1\right)}{2}}\right\} \\
& \left.-\sum_{i=1}^{N}\left\{W_{i j}\left[\frac{\frac{\delta y_{i}\left(y_{i}-1\right)}{2}\left[2\left(1+u_{i}\right)-\frac{\left(1+u_{i}\right)^{2}}{u_{i}}\right]+u_{i}\left[\delta y_{i}\left(1-n_{i}\right)+\frac{\delta n_{i}\left(n_{i}-1\right)}{2}\right]}{1+\frac{\delta y_{i}\left(y_{i}-1\right)}{2}\left[\frac{\left(1+u_{i}\right)^{2}}{u_{i}}\right]+\delta y_{i}\left(1-n_{i}\right)\left(1+u_{i}\right)+u_{i} \frac{\delta n_{i}\left(n_{i}-1\right)}{2}}\right]\right\}^{2}\right\}
\end{aligned}
$$

$$
\begin{aligned}
& \frac{\partial^{2} l\left(\delta, \beta, W_{i}\right)}{\partial \beta_{j} \partial \beta_{k}}=\frac{\partial^{2} l\left(\delta, \beta, W_{i}\right)}{\partial \beta_{k} \partial \beta_{j}} \\
& =-\sum_{i=1}^{N}\left[n_{i} W_{i j} W_{i k}\left(\frac{u_{i}\left(1+u_{i}\right)-u_{i}^{2}}{\left(1+u_{i}\right)^{2}}\right)\right]
\end{aligned}
$$$$
+\sum_{i=1}^{N}\left\{W_{\eta} W_{i k} \frac{\frac{\delta y_{i}\left(y_{i}-1\right)}{2}\left[-2+\frac{\left(1+u_{i}\right)^{2}}{u_{i}}\right]+u_{i}\left[\delta y_{i}\left(1-n_{i}\right)+\frac{\delta n_{i}\left(n_{i}-1\right)}{2}\right]}{1+\frac{\delta y_{i}\left(y_{i}-1\right)}{2}\left[\frac{\left(1+u_{i}\right)^{2}}{u_{i}}\right]+\delta y_{i}\left(1-n_{i}\right)\left(1+u_{i}\right)+u_{i} \frac{\delta n_{i}\left(n_{i}-1\right)}{2}}\right\}
$$$$
\left.-\sum_{i=1}^{N}\left\{W_{i j} W_{i k}\left[\frac{\frac{\delta y_{i}\left(y_{i}-1\right)}{2}\left[2\left(1+u_{i}\right)-\frac{\left(1+u_{i}\right)^{2}}{u_{i}}\right]+u_{i}\left[\delta y_{i}\left(1-n_{i}\right)+\frac{\delta n_{i}\left(n_{i}-1\right)}{2}\right]}{1+\frac{\delta y_{i}\left(y_{i}-1\right)}{2}\left[\frac{\left(1+u_{i}\right)^{2}}{u_{i}}\right]+\delta y_{i}\left(1-n_{i}\right)\left(1+u_{i}\right)+u_{i} \frac{\delta n_{i}\left(n_{i}-1\right)}{2}}\right]\right\}^{2}\right\}
$$

onde: $j \neq k$. 


$$
\begin{aligned}
& \frac{\partial^{2} l\left(\delta, \beta, W_{i}\right)}{\partial \delta \delta \beta_{0}}=\frac{\partial^{2} l\left(\delta, \underset{\sim}{\beta, W_{i}}\right)}{\partial \beta_{0} \partial \delta} \\
& =\sum_{i=1}^{N}\left\{\frac{\frac{y_{i}\left(y_{i}-1\right)}{2}\left[2\left(1+u_{i}\right)-\frac{\left(1+u_{i}\right)^{2}}{u_{i}}\right]+u_{i}\left[y_{i}\left(1-n_{i}\right)+\frac{n_{i}\left(n_{i}-1\right)}{2}\right]}{1+\frac{\delta y_{i}\left(y_{i}-1\right)}{2}\left[\frac{\left(1+u_{i}\right)^{2}}{u_{i}}\right]+\delta y_{i}\left(1-n_{i}\right)\left(1+u_{i}\right)+u_{i} \frac{\delta n_{i}\left(n_{i}-1\right)}{2}}\right\} \\
& -\sum_{i=1}^{N}\left\{\frac{\delta \frac{y_{i}\left(y_{i}-1\right)}{2}\left[2\left(1+u_{i}\right)-\frac{\left(1+u_{i}\right)^{2}}{u_{i}}\right]+u_{i}\left[y_{i}\left(1-n_{i}\right)+\frac{n_{i}\left(n_{i}-1\right)}{2}\right]\left[\frac{y_{i}\left(y_{i}-1\right)}{2}\left[\frac{\left(1+u_{i}\right)^{2}}{u_{i}}\right]+y_{i}\left(1-n_{i}\right)\left(1+u_{i}\right)+u_{i} \frac{n_{i}\left(n_{i}-1\right)}{2}\right]}{\left[\begin{array}{c}
\left.\delta_{i}\left(y_{i}-1\right)\left[\frac{\left(1+u_{i}\right)^{2}}{u_{i}}\right]+\delta_{i}\left(1-n_{i}\right)\left(1+u_{i}\right)+u_{i} \frac{\delta_{i}\left(n_{i}-1\right)}{2}\right]^{2} \\
2
\end{array}\right\}}\right\} \\
& \frac{\partial^{2} l\left(\delta, \beta, W_{i}\right)}{\partial \delta \partial \beta_{j}}=\frac{\partial^{2} l\left(\delta, \beta, W_{i}\right)}{\partial \beta_{j} \partial \delta} \\
& =\sum_{i=1}^{N}\left\{W_{i j} \frac{\frac{y_{i}\left(y_{i}-1\right)}{2}\left[2\left(1+u_{i}\right)-\frac{\left(1+u_{i}\right)^{2}}{u_{i}}\right]+u_{i}\left[y_{i}\left(1-n_{i}\right)+\frac{n_{i}\left(n_{i}-1\right)}{2}\right]}{1+\frac{\delta y_{i}\left(y_{i}-1\right)}{2}\left[\frac{\left(1+u_{i}\right)^{2}}{u_{i}}\right]+\delta y_{i}\left(1-n_{i}\right)\left(1+u_{i}\right)+u_{i} \frac{\delta n_{i}\left(n_{t}-1\right)}{2}}\right\} \\
& -\sum_{i=1}^{v}\left\{W_{i j} \frac{\delta \frac{y_{i}\left(y_{i}-1\right)}{2}\left[2\left(1+u_{i}\right)-\frac{\left(1+u_{i}\right)^{2}}{u_{i}}\right]+u_{i}\left[y_{i}\left(1-n_{i}\right)+\frac{n_{i}\left(n_{i}-1\right)}{2}\right]\left[\frac{y_{i}\left(y_{i}-1\right)}{2}\left[\frac{\left(1+u_{i}\right)^{2}}{u_{i}}\right]+y_{i}\left(1-n_{i}\right)\left(1+u_{i}\right)+u_{i} \frac{n_{i}\left(n_{i}-1\right)}{2}\right]}{\left[1+\frac{\delta_{i}\left(y_{i}-1\right)}{2}\left[\frac{\left(1+u_{i}\right)^{2}}{u_{i}}\right]+\delta_{i}\left(1-n_{i}\right)\left(1+u_{i}\right)+u_{i} \frac{\delta_{(}(n-1)}{2}\right]^{2}}\right\}
\end{aligned}
$$

Utilizamos o critério de mínima informação (AIC), discutido no capítulo 5, para uma discriminação Clássica dos modelos na presença de covariáveis. 


\subsection{2 - Análise Bayesiana dos modelos}

Para o modelo de regressão logística, dado em 6.4, assumimos as seguintes distribuições a priori para os parâmetros:

(i) $\beta_{0} \sim N\left(\mu_{0}, \sigma_{0}^{2}\right) ; \mu_{0}, \sigma_{0}^{2}$ conhecidos

(ii) $\beta_{j} \sim N\left(\mu_{j}, \sigma_{j}^{2}\right) ; \mu_{j}, \sigma_{j}^{2}$ conhecidos

onde: $j=1, \ldots, m$

Considerando que as distribuições a priori para os parâmetros são independentes, a função densidade conjunta a priori para $\beta_{0}, \beta_{1} \ldots, \beta_{m}$ é dada por,

$$
\pi\left(\beta_{0}, \beta_{1}, \ldots, \beta_{m}\right) \times \exp \left\{-\frac{1}{2 \sigma_{0}^{2}}\left(\beta_{0}-\mu_{0}\right)^{2}\right\} \exp \left\{-\frac{1}{2 \sigma_{0}^{2}}\left(\beta_{1}-\mu_{1}\right)^{2}\right\} \ldots \exp \left\{-\frac{1}{2 \sigma_{0}^{2}}\left(\beta_{m}-\mu_{m}\right)^{2}\right\}
$$

Combinando as expressões dadas em (6.7) e (6.16), encontramos a distribuição a posteriori conjunta para $\beta_{i}, \beta_{1} \ldots, \beta_{m}$, dada por,

$$
\begin{aligned}
& \pi\left(\beta_{0}, \beta_{1}, \ldots, \beta_{m} / W_{i}, y, n\right) \times \exp \left\{-\frac{1}{2 \sigma_{0}^{2}}\left(\beta_{0}-\mu_{0}\right)^{2}\right\} \ldots \exp \left\{-\frac{1}{2 \sigma_{0}^{2}}\left(\beta_{m}-\mu_{m}\right)^{2}\right\} \\
& \exp \left\{\beta_{0} \sum_{i=1}^{N} y_{i}+\sum_{i=1}^{N}\left[y_{i} \sum_{i=1}^{m} \beta_{i} W_{i j}\right]-\sum_{i=1}^{N}\left[n_{i} \log \left(1+e^{\sum_{j=0}^{m} \beta_{j} W_{i j}}\right)\right]\right\} \\
& \text { onde: } W_{i}=\left(W_{i 1}, \ldots, W_{i m}\right), y=\left(y_{1}, \ldots, y_{N}\right) \text { e } n=\left(n_{1}, \ldots, n_{N}\right) .
\end{aligned}
$$

Logo, as distribuições condicionais a posteriori para $\beta_{0}$ e $\beta_{j}, j=1, \ldots, m$, necessárias para o uso dos métodos MCMC, são dadas por, 


$$
\begin{gathered}
\pi\left(\beta_{0} / \beta_{1}, \ldots, \beta_{m}, W_{i}, y, n\right) \propto \exp \left\{-\frac{1}{2 \sigma_{0}^{2}}\left(\beta_{0}-\mu_{0}\right)^{2}\right\} \exp \left\{\beta_{0} \sum_{i=1}^{N} y_{i}-\sum_{i=1}^{N}\left[n_{i} \log \left(1+e^{\sum_{j=0}^{m} \beta_{j} W_{i j}}\right]\right)\right\} \\
\pi\left(\beta_{j} / \beta_{0}, \beta_{i}, \ldots, \beta_{j-1}, \beta_{j+1}, \ldots, \beta_{m}, W_{i} y, n\right) \propto \exp \left\{-\frac{1}{2 \sigma_{j}^{2}}\left(\beta_{j}-\mu_{j}\right)^{2}\right\} \exp \left\{\beta_{j} \sum_{i=1}^{N} y_{i} W_{i j}-\sum_{i=1}^{N}\left[n_{i} \log \left\{1+e^{\sum_{j=0}^{m} \beta_{j} W_{i j}}\right)\right]\right\}
\end{gathered}
$$

Para o modelo Binomial Correlacionado com correlações homogêneas, dado em (6.5), assumimos para os parâmetros $\beta_{0} \mathrm{e} \beta_{j}, j=1, \ldots, m$, as mesmas distribuições a priori dadas em (6.15). Com relação ao parâmetro de correlação $\delta$, assumimos uma distribuição a priori Uniforme $(a, b)$, onde $a$ e $b$ são conhecidos.

Assumindo independência a priori para os parâmetros $\delta, \beta_{0}$ e $\beta, j=1, \ldots, m$, temos que a função densidade conjunta a priori do modelo Binomial Correlacionado com correlações homogêneas é proporcional a do modelo de regressão logística, dada em (6.16). Assim, combinando as expressões (6.11) e (6.16), encontramos a distribuição a posteriori conjunta para $\delta, \beta_{0}$ e $\beta_{j}, j=1, \ldots, m$, dada por,

$$
\begin{aligned}
& \pi\left(\delta, \beta_{0}, \beta_{1}, \ldots, \beta_{m} / W_{i} y, n\right) \propto \exp \left\{-\frac{1}{2 \sigma_{0}^{2}}\left(\beta_{0}-\mu_{0}\right)^{2}\right\} \exp \left\{-\frac{1}{2 \sigma_{0}^{2}}\left(\beta_{m}-\mu_{m}\right)^{2}\right\} \\
& \exp \left\{\beta_{i} \sum_{i=1}^{N} y_{i}+\sum_{i=1}^{N}\left[y_{i} \sum_{i=1}^{m} \beta W_{i j}\right]-\sum_{i=1}^{N}\left[n_{i} \log \left(1+e^{\sum_{i=0}^{m} \beta_{j} W_{i j}}\right)\right]+\sum_{i=1}^{N}\left[\log \left(A_{i}\left(n_{i}, y_{i}, \delta, \beta, W_{i}\right)\right)\right]\right\}
\end{aligned}
$$

onde: $W_{i}=\left(W_{i 1}, \ldots, W_{i m}\right), y=\left(y_{1}, \ldots, y_{N}\right)$ e $n=\left(n_{1}, \ldots, n_{N}\right)$.

A partir de (6.19), encontramos as distribuições condicionais a posteriori para $\delta, \beta_{0}$ e $\beta_{j}, j=1, \ldots, m$, que são dadas por, 


$$
\begin{aligned}
& \left.\pi\left(\delta / \beta_{0}, \beta_{1}, \ldots, \beta_{m}, W_{i}, y, n\right) \propto \exp \sum_{i=1}^{N}\left[\log \left(A_{i}\left(n_{i}, y_{i}, \delta, \beta_{\sim}, W_{i}\right)\right)\right]\right\} \\
& \pi\left(\beta_{0} / \delta, \beta_{1}, \ldots, \beta_{m}, W_{i}, y, n\right) \propto \exp \left\{-\frac{1}{2 \sigma_{0}^{2}}\left(\beta_{0}-\mu_{0}\right)^{2}\right\} \\
& \exp \left\{\beta_{i} \sum_{i=1}^{N} y_{i}-\sum_{i=1}^{N} n_{i} \log \left(1+e^{\sum_{j=0}^{m} \beta_{j} W_{i j}}\right)+\sum_{i=1}^{N}\left[\log \left(A_{i}\left(n_{i}, y_{i}, \delta, \beta, W_{i}\right)\right)\right]\right\} \\
& \pi\left(\beta_{j} / \delta, \beta_{0}, \beta_{1}, \ldots, \beta_{j-1}, \beta_{j i 1}, \ldots, \beta_{m}, W_{i}, y, n\right) \propto \exp \left\{-\frac{1}{2 \sigma_{j}^{2}}\left(\beta_{j}-\mu_{j}\right)^{2}\right\} \\
& \exp \left\{\beta_{j} \sum_{i=1}^{N} y_{i} W_{i j}-\sum_{i=1}^{N} n_{i} \log \left(1+e^{\sum_{j=0}^{m} \beta_{j} W_{i j}}\right)+\sum_{i=1}^{N}\left[\log \left(A_{i}\left(n_{i}, y_{i}, \delta, \beta, W_{i}\right)\right)\right]\right\}
\end{aligned}
$$

Para o modelo Binomial Correlacionado com correlações diferentes, dado em (6.6), assumimos para os parâmetros $\beta_{0}$ e $\beta_{j}, j=1, \ldots, m$, as mesmas distribuições a priori dadas em (6.15) e consideramos distribuições a priori Uniforme $(a, b)$, com $a$ e $b$ conhecidos, para $\delta_{i}$, $i=1, \ldots, N$.

Desenvolvendo uma análise Bayesiana para este modelo seguindo o mesmo procedimento usado nos modelos anteriores, encontramos as distribuições condicionais a posteriori para os parâmetros $\delta_{i}, i=1, \ldots, N, \beta_{0}$ e $\beta_{j}, j=1, \ldots, m$. Para $\beta_{0}$ e $\beta_{j}$, as distribuições condicionais são as mesmas dadas em (6.20), apenas substituindo $\delta$ por $\delta_{t}$. Com relação a $\delta_{i}, i=1, \ldots, N$, a distribuição condicional é dada por,

$$
\pi\left(\delta_{i} / \delta_{1}, \ldots, \delta_{i-1}, \delta_{i+1}, . ., \delta_{N}, \beta_{0}, \beta_{1}, \ldots, \beta_{m}, W_{i}, y, n\right) \propto \exp \left\{\log \left[A_{i}\left(n_{i}, y_{i}, \delta, \beta_{\sim}, W_{i}\right)\right]\right\}
$$


Observando as distribuições condicionais dadas em (6.18), (6.20) e (6.21), verificamos que todas elas não apresentam a formas de distribuições conhecidas. Portanto, utilizamos o algoritmo Metropolis Hastings para realizar a simulação dos parâmetros em todos os modelos considerados neste trabalho na presença de covariáveis.

Utilizamos o fator de Bayes, discutido no capitulo 5, para uma discriminação Bayesiana dos modelos na presença de covariáveis. Escolhemos a aproximação de Monte Carlo, dada em (5.5), para estimar a verossimilhança marginal dos modelos. Para os modelos de regressão logística, Binomial Correlacionado com correlações homogêneas e Binomial Correlacionado com correlações diferentes, representados respectivamente por $M_{5}, M_{6}$ e $M_{7}$, as estimativas de Monte Carlo da verossimilhança marginal são dadas por,

1) Modelo de regressão logistica

$$
\hat{p}\left(y / M_{5}\right)=\frac{1}{S} \sum_{s=1}^{S}\left\{\prod_{i=1}^{N}\left[\left(\begin{array}{l}
n_{i} \\
y_{i}
\end{array}\right)\left(p_{i}^{(s)}\right)^{y_{i}}\left(1-p_{i}^{(s)}\right)^{n_{i}-y_{i}}\right]\right\}
$$

2) Modelo Binomial Correlacionado com correlações homogêneas

$$
\hat{p}\left(y / M_{6}\right)=\frac{1}{S} \sum_{s=1}^{S}\left\{\prod_{i=1}^{N}\left[\left(\begin{array}{l}
n_{i} \\
y_{i}
\end{array}\right)\left(p_{i}^{(s)}\right)^{y_{1}}\left(1-p_{i}^{(s)}\right)^{n_{i}-y_{i}}\left\{1+\frac{\delta^{(s)}}{2 p_{i}^{(s)}\left(1-p_{i}^{(s)}\right)}\left[\left(y_{i}-n_{i} p_{i}^{(s)}\right)^{2}+y_{i}\left(2 p_{i}^{(s)}-1\right)-n_{i}\left(p_{i}^{(s)}\right)^{2}\right]\right\}\right]\right\}
$$

3) Modelo Binomial Correlacionado com correlações diferentes

$$
\hat{p}\left(y / M_{7}\right)=\frac{1}{s} \sum_{s=1}^{s}\left\{\prod_{i=1}^{N}\left[\left(\begin{array}{l}
n_{i} \\
y_{i}
\end{array}\right)\left(p_{i}^{(s)}\right)^{y_{i}}\left(1-p_{i}^{(s)}\right)^{n_{i}-y_{i}}\left\{1+\frac{\delta_{i}^{(s)}}{2 p_{i}^{(s)}\left(1-p_{i}^{(s)}\right)}\left[\left(y_{i}-n_{i} p_{i}^{(s)}\right)^{2}+y_{i}\left(2 p_{i}^{(s)}-1\right)-n_{i}\left(p_{i}^{(s)}\right)^{2}\right]\right\}\right]\right\}
$$

onde $S$ é o tamanho da amostra obtida por simulação. 


\section{Capítulo 7: Aplicações Numéricas}

Neste capítulo, apresentamos três exemplos numéricos para ilustrar a metodologia proposta nos capítulos anteriores desta dissertação.

Nas seções 7.1 e 7.2, analisamos dois conjuntos de dados de contagens de sucessos, onde não temos a presença de covariáveis. Assim, para verificar se a variabilidade extra-Binomial está presente nos dados, utilizamos nesses exemplos os modelos discutidos nos capítulos 2,3 e 4 deste trabalho. Na primeira aplicação, descrita na seção 7.1 , analisamos dados de genética, e na segunda aplicação, descrita na seção 7.2 , analisamos dados de um jogador da liga americana de basquete.

$\mathrm{Na}$ seção 7.3, apresentamos um exemplo onde covariáveis estão associadas às observações. Os dados analisados se originam de um experimento agronômico e a metodologia proposta no capítulo 6 é utilizada.

Os resultados Clássicos foram obtidos utilizando o "software" Sas e os resultados Bayesianos utilizando o "software" Matlab.

\section{1 - Primeira aplicação: dados genéticos}

Nesta aplicação, consideramos os dados genéticos introduzidos por Skellam (1948), onde o objetivo é a associação secundária de cromossomos numa espécie de couve flor e repolho, chamada Brassica.

As unidades observadas são 337 núcleos, sendo que em cada núcleo existem 3 pares de cromossomos durante a meiose. Cada par pode ou não mostrar uma associação entre esses cromossomos, ou seja em cada núcleo podemos ter $0,1,2$ ou 3 pares de cromossomos associados. As frequêencias observadas com $0,1,2$ e 3 pares associados nesses núcleos são dadas por $32,103,122$ e 80 , respectivamente.

Observamos que neste exemplo, a variável $Y_{i}$ representa $o$ número de pares de cromossomos associados no i-ésimo núcleo, $i=1, \ldots, 337$. Assumimos para $Y_{i}$ os modelos 
Binomial, Beta-Binomial, Binomial Correlacionado e mistura de duas distribuições Binomiais e apresentamos os resultados obtidos na seções $7.1 .1,7.1 .2,7.1 .3$ e 7.1 .4 , respectivamente. A seção 7.1.5 é dedicada a uma comparação de todos os modelos com base nos critérios discutidos no capítulo 5 .

\subsection{1 - Aplicação do modelo Binomial}

No apèndice $D$, descrevemos uma análise estatistica para o modelo Binomial, utilizando os métodos Clássicos e Bayesianos. Com base nessas análises, aplicamos o modelo Binomial para os dados genéticos e nesta seção apresentamos os resultados obtidos.

Assumindo uma distribuição $\operatorname{Binomial}(3, p)$ para $Y_{i}, i=1, \ldots, 337$, estamos considerando que a probabilidade de associação dos cromossomos é constante para qualquer par em algum núcleo.

A tabela 7.1 apresenta os resultados da análise Clássica, onde temos a estimativa de máxima verossimilhança do parâmetro $p$ e seu respectivo intervalo de confiança obtido por aproximação assintótica.

Tabela 7.1 - Resultados da análise Clássica do modelo Binomial para os dados genéticos

\begin{tabular}{ccc}
\hline Parâmetro & EMV & Int. Conf. $(95 \%)$ \\
\hline$p$ & 0,5806 & $(0,5502 ; 0,6110)$ \\
\hline
\end{tabular}

A partir de EMV do parâmetro $p$, dado na tabela 7.1 , encontramos a variabilidade esperada para os dados genéticos assumindo o modelo Binomial, dada por $n_{i} \hat{p}(1-p)=0,7305$. Contudo, essa variabilidade esperada apresenta um valor menor do que a variabilidade observada, dada por $\sum_{i=1}^{N} \frac{\left(y_{i}-n_{i} \hat{p}\right)^{2}}{N}=0,8588$. Este fato motiva o uso de outros modelos para ajustar os dados genéticos. 
Na tabela 7.2 encontramos as quantidades a posteriori de interesse (média, desvio padrão e intervalo de credibilidade $(95 \%)$ ) para o parâmetro $p$ obtidos na análise Bayesiana.

Tabela 7.2 - Sumários a posteriori do modelo Binomial para os dados genéticos

\begin{tabular}{cccc}
\hline Parâmetro & Média & DP & Int. Cred. (95\%) \\
\hline$p$ & 0,5805 & 0,0155 & $(0,5501 ; 0,6108)$ \\
\hline
\end{tabular}

Comparando os resultados Clássicos e Bayesianos do modelo Binomial, descritos nas tabelas 7.1 e 7.2 , respectivamente, observamos que as estimativas pontuais e por intervalo do parâmetro $p$ praticamente coincidiram.

\subsection{2 - Aplicação do modelo Beta-Binomial}

Na tabela 7.3 apresentamos os resultados da análise Clássica do modelo Beta-Binomial, considerando os dados genéticos. Esses resultados foram obtidos utilizando a metodologia proposta na seção 2.2 deste trabalho.

Tabela 7.3 - Resultados da análise Clássica do modelo Beta-Binomial para os dados genéticos.

\begin{tabular}{ccc}
\hline Parâmetro & EMV & Int. Conf. $(95 \%)$ \\
\hline$\alpha$ & 6,1198 & $(0,9563 ; 11,2833)$ \\
$\beta$ & 4,4186 & $(0,6945 ; 8,1427)$ \\
\hline
\end{tabular}

$\mathrm{Na}$ análise Bayesiana do modeio Beta-Binomial para os dados genéticos, assumimos as distribuições a priori dadas em (2.15) com $a_{1}=90, b_{1}=15, a_{2}=70$ e $b_{2}=16$.

Baseado nas distribuições condicionais a posteriori dadas em (2.18), utilizamos o algoritmo Metropolis-Hastings para a simulação dos parâmetros. Visando diminuir a autocorrelação das amostras geradas por esse algoritmo, geramos várias cadeias com grande quantidade de pontos e selecionamos sistematicamente valores dentro de cada cadeia, formando novas amostras. Foram geradas 5 cadeias de 8000 pontos para cada parâmetro. Para cada cadeia 
desprezamos os 4000 valores iniciais e selecionamos dos restantes, 1 em cada 20 valores, formando 5 amostras com 200 valores.

Consideramos os seguintes valores iniciais para as cadeias dos parâmetros $\alpha$ e $\beta$ :

$$
\begin{aligned}
& \alpha=\left[\begin{array}{lllll}
4,5 & 5,0 & 5,5 & 6,0 & 6,5
\end{array}\right] \\
& \beta=\left[\begin{array}{lllll}
3 & 3,5 & 4 & 4,5 & 5
\end{array}\right]
\end{aligned}
$$

A tabela 7.4 nos mostra os resumos a posteriori dos parâmetros $\alpha$ e $\beta$. Apresentamos para cada parâmetro, a média, o desvio padrão, o intervalo de credibilidade( $95 \%$ ), o fator de redução e escala (GR) do critério de convergência de Gelman \& Rubin e a porcentagem de aceitação dos valores gerados pelo algoritmo Metropolis-Hastings.

Tabela 7.4 - Sumários a posteriori do modelo Beta-Binomial para os dados genéticos.

\begin{tabular}{cccccc}
\hline Parâmetro & Média & DP & Int. Cred. (95\%) & GR & Tac \\
\hline$\alpha$ & 6,0275 & 0,4865 & $(5,0826 ; 6,9582)$ & 1,0001 & 54,38 \\
$\beta$ & 4,3629 & 0,3788 & $(3,6652 ; 5,1431)$ & 1,0001 & 51,65 \\
\hline
\end{tabular}

Comparando os resultados das tabelas 7.3 e 7.4 , observamos que as médias a posteriori de $\alpha$ e $\beta$ ficaram bem próximas dos EMV desses parâmetros, ao passo que os intervalos de credibilidade de $\alpha$ e $\beta$ apresentaram uma precisão maior que os intervalos de confiança, obtidos por aproximação assintótica.

Para ambos os parâmetros tivemos uma razoável taxa de aceitação dos valores gerados pelo algoritmo Metropolis-Hastings e a convergência foi atingida pelo critério de Gelman \& Rubin, pois os valores do fator de redução e escala se aproximaram de 1 .

Outra forma de verificarmos a convergência dos parâmetros, é através dos gráficos dados a seguir que nos mostram as trajetórias das cadeias ao longo das iterações.

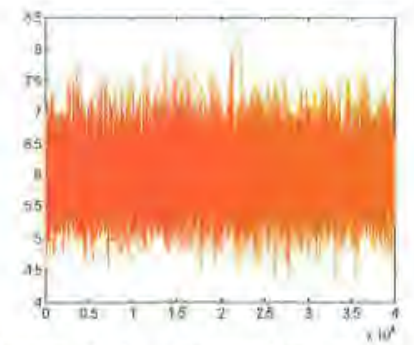

Figura 3.1 - Iterações para $\alpha$

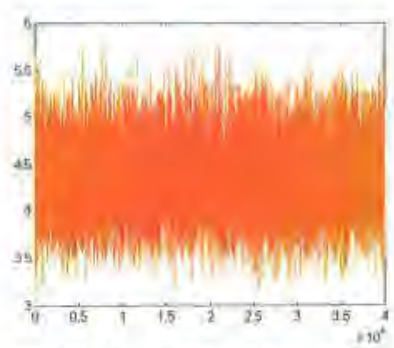

Figura 3.2 - Iterações para $\beta$ 
Observando as figuras 3.1 e 3.2 , verificamos que após um periodo inicial as diferentes cadeias se aproximam de um ponto de equilibrio.

A seguir apresentamos as figuras 3.3 e 3.4 , que são histogramas referentes aos 1000 valores obtidos após a seleção das amostras dos parâmetros $\alpha$ e $\beta$. Esses histogramas representam aproximações das densidades a posteriori marginais desses parâmetros.

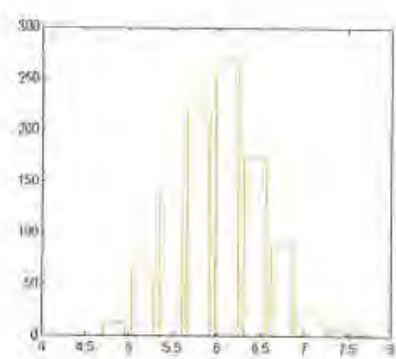

Figura 3.3 - Aproximação da densidade a posteriori marginal de $\alpha$

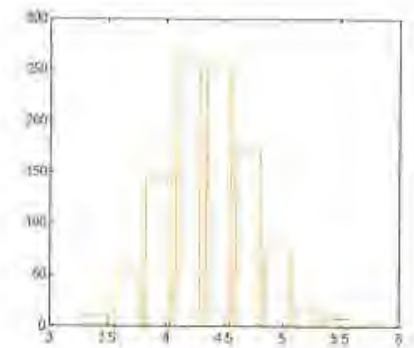

Figura 3.4 - Aproximação da densidade a posteriori marginal de $\beta$

\subsection{3 - Aplicação do modelo Binomial Correlacionado}

Na tabela 7.5 apresentamos os resultados da análise Clássica do modelo Binomial Correlacionado, para os dados genéticos. Esses resultados foram obtidos utilizando a metodologia proposta na seção 3.2 deste trabalho.

Tabela 7.5 - Resultados da análise Clássica do modelo Binomial Correlacionado para os dados genéticos.

\begin{tabular}{ccc}
\hline Parâmetro & EMV & Int. Conf. $(95 \%)$ \\
\hline$p$ & 0,5809 & $(0,5480 ; 0,6139)$ \\
$\delta$ & 0,0883 & $(0,0214 ; 0,1553)$ \\
\hline
\end{tabular}

$\mathrm{Na}$ análise Bayesiana do modelo Binomial Correlacionado para os dados genéticos, assumimos inicialmente as distribuições a priori dadas em (3.8) com $a=0, b=0,15, c=30 \mathrm{e}$ 
$d=22$. Posteriormente, assumimos as distribuições a priori dadas em (3.12) com $a=0$, $b=0,15, c=0$ e $d=1$.

Para a simulação dos parâmetros, utilizamos as distribuições condicionais a posteriori dadas em (3.11) e (3.14). Em ambos os casos geramos 4 cadeias de 10000 pontos para cada parâmetro, através do algoritmo Metropolis-Hastings. Para cada cadeia descartamos os 5000 valores iniciais e consideramos as iterações $5010 .^{\mathrm{a}}, 5020^{\mathrm{a}}, \ldots, 10000^{\mathrm{a}}$, totalizando assim uma amostra final de 2000 pontos.

Consideramos os seguintes valores iniciais para as cadeias dos parâmetros $\delta$ e $p$ :

$$
\begin{aligned}
& p=\left[\begin{array}{llll}
0,5 & 0,54 & 0,58 & 0,62
\end{array}\right] \\
& \delta=\left[\begin{array}{llll}
0,1 & 0,2 & 0,3 & 0,4
\end{array}\right]
\end{aligned}
$$

Apresentamos nas tabelas 7.6 e 7.7 os resultados obtidos na análise Bayesiana do modelo Binomial Correlacionado, considerando as duas estratégias descritas na seção 3,3.

Tabela 7.6 - Sumários a posteriori do modelo Binomial Correlacionado para os dados genéticos, considerando a primeira estratégia.

\begin{tabular}{cccccc}
\hline Parâmetro & Média & DP & Int. Cred. (95\%) & GR & Tac \\
\hline$p$ & 0,5810 & 0,0159 & $(0,5496 ; 0,6112)$ & 1,0001 & 95,21 \\
$\delta$ & 0,0865 & 0,0313 & $(0,0246 ; 0,142)$ & 1,0000 & 65,86 \\
\hline
\end{tabular}

Tabela 7.7 - Sumários a posteriori do modelo Binomial Correlacionado para os dados genéticos, considerando a segunda estratégia.

\begin{tabular}{cccccc}
\hline Parâmetro & Média & DP & Int. Cred. (95\%) & GR & Tac \\
\hline$p$ & 0,5813 & 0,0167 & $(0,5472 ; 0,6133)$ & 1,0000 & 94,82 \\
$\delta$ & 0,0872 & 0,0311 & $(0,024 ; 0,141)$ & 1,0000 & 65,37 \\
\hline
\end{tabular}

Observamos que as tabelas 7.6 e 7.7 apresentam resultados bem parecidos, indicando que não existe diferenças significativas entre as duas estratégias de distribuições a priori para os parâmetros $\delta$ e $p$, quando utilizamos os dados genéticos. Comparando os resultados das tabelas 7.6 e 7.7 com os resultados da análise Clássica do modelo Binomial Correlacionado dados na tabela 7.5, observamos que as estimativas pontuais e por intervalo ficaram bem próximas. Outra caracteristica interessante é que tanto o intervalo de confiança como os dois intervalos de 
credibilidade obtidos para o parâmetro $\delta$, não contém o número 0 , o que é uma forte evidência que existe uma correlação positiva entre as variáveis binárias de $Y_{i}$,

Para os parâmetros $\delta$ e $p$ tivemos uma excelente taxa de aceitação dos valores gerados pelo algoritmo Metropolis-Hastings e a convergência foi atingida pelo critério de Gelman \& Rubin, pois os valores do fator de redução e escala se aproximaram de 1 ,

Nas figuras 3.5 e 3.6 apresentamos as trajetórias das cadeias ao longo das iterações dos parâmetros e nas figuras 3.7 e 3.8 apresentamos aproximações das densidades a posteriori marginais de $\delta$ e $p$, considerando a primeira estratégia.

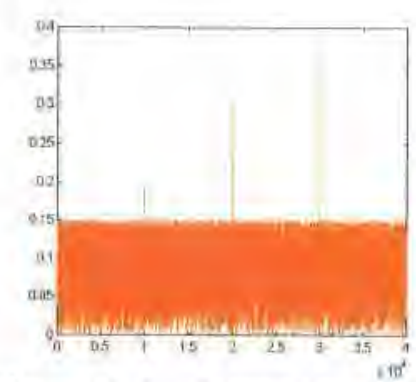

Figura 3.5 - Iterações para $\delta$

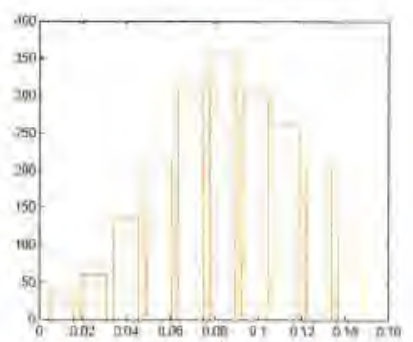

Figura 3.7 - Aproximação da densidade a posteriori marginal de $\delta$

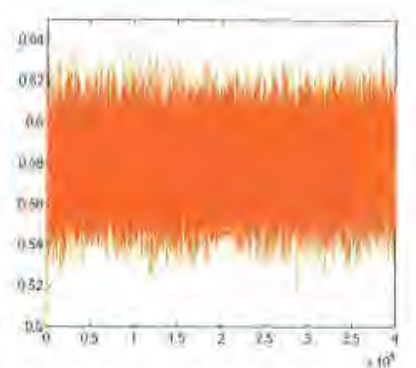

Figura 3.6 - Iterações para $p$

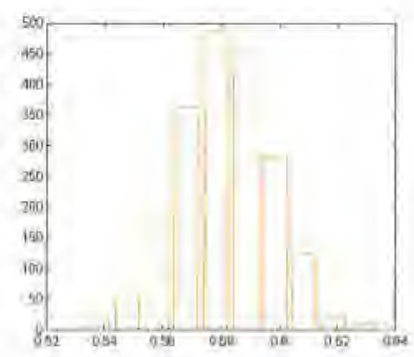

Figura 3.8 - Aproximação da densidade a posteriori marginal de $p$

\subsection{4 - Aplicação do modelo de mistura de duas distribuições Binomiais}

Na tabela 7.8 apresentamos os resultados da análise Clássica do modelo de mistura de duas distribuições Binomiais, considerando os dados genéticos. Esses resultados foram obtidos utilizando a metodologia proposta na seção 4.2 deste trabalho. 
Tabela 7.8 - Resultados da análise Clássica do modelo de mistura de duas distribuições Binomiais para os dados genéticos.

\begin{tabular}{ccc}
\hline Parâmetro & EMV & Int. Conf. (95\%) \\
\hline$\theta_{1}$ & 0,5138 & $(0,3738 ; 0,6538)$ \\
$\theta_{2}$ & 0,8941 & $(0,3644 ; 1)$ \\
$\pi$ & 0,8242 & $(0,2911 ; 1)$ \\
\hline
\end{tabular}

$\mathrm{Na}$ análise Bayesiana do modelo de mistura de duas distribuiçōes Binomiais para os dados genéticos, assumimos as distribuições a priori dadas em (4.10) com $a_{1}=30, b_{1}=30$, $a_{2}=20, b_{2}=2, c=18$ e $d=4$.

Baseados nas distribuições condicionais a posteriori dadas em (4.13), utilizamos o algoritmo Gibbs Sampling para a simulação dos parâmetros. Para cada parâmetro geramos 5 cadeias de 10000 pontos, sendo que para cada cadeia descartamos as primeiras 5000 iterações e

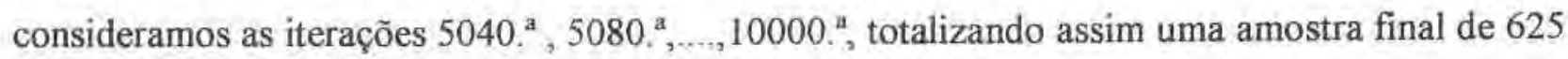
pontos.

Consideramos os seguintes valores iniciais para as cadeias dos parâmetros $\theta_{1}, \theta_{2}$ e $\pi$ :

$$
\begin{aligned}
& \theta_{1}=\left[\begin{array}{lllll}
0,1 & 0,3 & 0,5 & 0,7 & 0,9
\end{array}\right] \\
& \theta_{2}=\left[\begin{array}{lllll}
0,1 & 0,3 & 0,5 & 0,7 & 0,9
\end{array}\right] \\
& \pi=\left[\begin{array}{lllll}
0,1 & 0,3 & 0,5 & 0,7 & 0,9
\end{array}\right]
\end{aligned}
$$

Apresentamos nas tabela 7.9 os resultados obtidos na análise Bayesiana do modelo de mistura de duas distribuições Binomiais.

Tabela 7.9 - Sumários a posteriori do modelo de mistura de duas distribuições Binomiais para os dados genéticos.

\begin{tabular}{ccccc}
\hline Parâmetro & Média & DP & Int. Cred. (95\%) & GR \\
\hline$\theta_{1}$ & 0,5162 & 0,0237 & $(0,4675 ; 0,5611)$ & 1,0005 \\
$\theta_{2}$ & 0,9053 & 0,0486 & $(0,7996 ; 0,9825)$ & 1,0002 \\
$\pi$ & 0,8341 & 0,0526 & $(0,7097 ; 0,9236)$ & 1,0012 \\
\hline
\end{tabular}

Comparando os resultados Clássicos e Bayesianos dados nas tabelas 7.8 e 7.9 , respectivamente, observamos que as estimativas pontuais de $\theta_{1}, \theta_{2}$ e $\pi$ ficaram bem próximas, 
ao passo que os intervalos de credibilidade desses parâmetros apresentaram uma precisão maior que os intervalos de confiança, obtidos por aproximação assintótica.

Para os parâmetros $\theta_{1}, \theta_{2}$ e $\pi$ a convergência foi atingida pelo critério de Gelman \& Rubin, pois os valores do fator de redução e escala se aproximaram de 1 .

Nas figuras $3.9,3.10$ e 3.11 apresentamos as trajetórias das cadeias ao longo das iterações dos parâmetros e nas figuras $3.12,3.13$ e 3.14 apresentamos aproximações das densidades a posteriori marginais de $\theta_{1}, \theta_{2}$ e $\pi$.

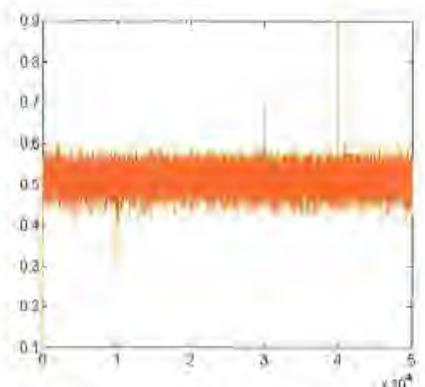

Figura 3.9 - Iterações para $\theta_{1}$

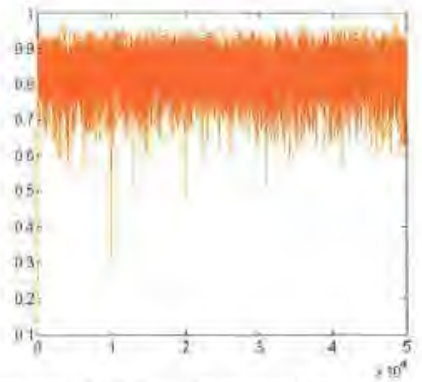

Figura 3.11 - Iterações para $\pi$

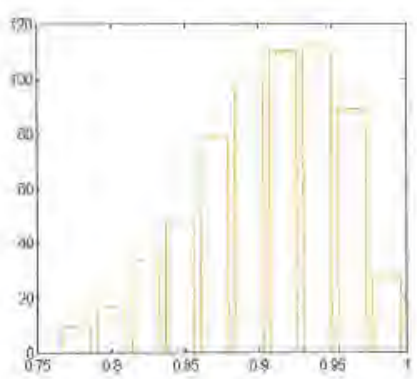

Figura 3.13 - Aproximação da densidade a posteriori marginal de $\theta_{2}$

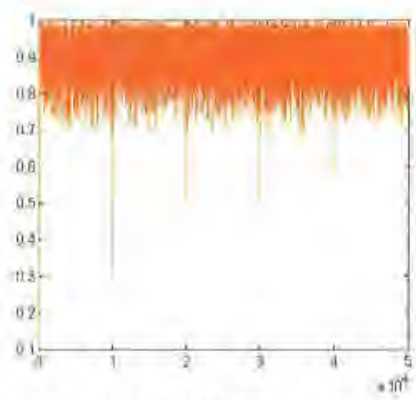

Figura 3.10 - Iterações para $\theta_{2}$

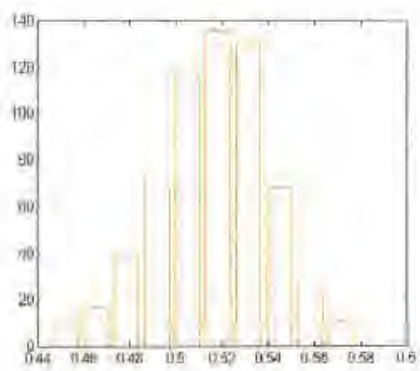

Figura 3.12 - Aproximação da densidade a posteriori marginal de $\theta_{1}$

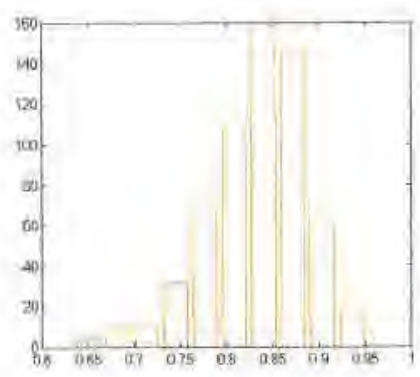

Figura 3.14 - Aproximação da densidade a posteriori marginal de $\pi$ 


\subsection{5 - Comparação dos modelos}

Para uma discriminação Clássica dos modelos Binomial, Beta-Binomial, Binomial Correlacionado e mistura de duas distribuições Binomiais, utilizamos o critério de mínima informação (AIC), discutido na seção 5.1

Apresentamos na tabela 7.10 os resultados do AIC obtidos com os dados genéticos.

Tabela 7.10 - Discriminação Clássica dos modelos para os dados genéticos

\begin{tabular}{cc}
\hline Modelo & AIC \\
\hline Binomial & 882,7731 \\
Beta-Binomial & 877,6259 \\
Binomial Correlacionado & 877,4859 \\
Mistura de 2 distribuições Binomiais & 878,8692 \\
\hline
\end{tabular}

Observamos que não existe diferenças significativas entre os valores do critério de minima informação dos modelos Beta-Binomial, Binomial Correlacionado e mistura de duas distribuições Binomiais, ao passo que esses valores foram inferiores ao AIC obtido considerando - modelo Binomial. Assim, na análise Clássica, os modelos Beta-Binomial, Binomial Correlacionado e mistura de duas distribuições Binomiais tiveram um melhor ajuste dos dados genéticos do que o modelo Binomial, indicando a presença da variabilidade extra-Binomial nesse conjunto de dados.

A discriminação Bayesiana dos modelos usados para ajustar os dados genéticos foi baseada na metodologia proposta na seção 5.2 deste trabalho, que se refere a verossimilhança marginal e ao fator de Bayes.

Para o modelo Binomial, determinamos o valor exato da verossimilhança marginal utilizando a expressão dada em (5.12). O valor encontrado foi $P\left(\underset{\sim}{y / M_{1}}\right)=0,2149 \times 10^{-192}$.

Com relação ao modelo Beta-Binomial, aproximamos a verossimilhança marginal utilizando o estimador de Monte Carlo dado na expressão (5.6) e o estimador de Laplace dado na expressão (5.10). As estimativas encontradas foram: $\stackrel{x}{P}\left(y / M_{2}\right)=1,4361 \times 10^{-190}$ (Monte Carlo) e $\hat{I}_{M_{2}}=0,7628 \times 10^{-190}$ (Laplace). 
Encontramos estimativas de Monte Carlo para a verossimilhança marginal do modelo Binomial Correlacionado, considerando as duas estratégias discutidas na seção 3.3. Os resultados obtidos utilizando a expressão dada em (5.7) foram: $\hat{P}\left(\underset{\sim}{y /} M_{31}\right)=1,1058 \times 10^{-190}$ (primeira estratégia) e $P\left(y / M_{32}\right)=1_{2} 0839 \times 10^{-190}$ (segunda estratégia).

Para o modelo de mistura de duas distribuições Binomiais, encontramos uma estimativa de Monte Carlo da verossimilhança marginal utilizando a expressão dada em (5.8). O valor encontrado foi $\hat{P}\left(y / M_{4}\right)=1,6122 \times 10^{-190}$.

Comparando as estimativas da verossimilhança marginal encontradas para os modelos Beta-Binomial, Binomial Correlacionado e mistura de duas distribuições Binomiais, verificamos que todas elas ficaram bem proximas, indicando que na análise Bayesiana esses modelos tiveram um comportamento semelhante no ajuste dos dados genéticos.

Utilizamos o fator de Bayes dado em (5.4), para comparar cada uma dessas estimativas da verossimilhança marginal com o valor da verossimilhança marginal exata do modelo Binomial. Na tabela 7.11 apresentamos os resultados obtidos, considerando em todos os casos a verossimilhança marginal exata do modelo Binomial como o denominador do fator de Bayes.

Tabela 7.11 - Discriminação Bayesiana dos modelos para os dados genéticos

\begin{tabular}{cc}
\hline Modelo Comparados & Fator de Bayes \\
\hline Beta-Binomial (MCMC) x Binomial & 668,26 \\
Beta-Binomial (Laplace) x Binomial & 354,95 \\
Binomial Correl. (Estratégia 1) x Binomial & 514,56 \\
Binomial Correl. (Estratégia 2) x Binomial & 504,37 \\
Mistura de 2 dist. Binomiais x Binomial & 750,21 \\
\hline
\end{tabular}

Observamos que em todos os casos, o fator de Bayes apresentou valores maiores que 300 , Assim, de acordo com a tabela 5.1 (interpretação do fator de Bayes), temos fortes evidências contra o modelo Binomial no ajuste dos dados genéticos.

Aplicamos também para este conjunto de dados o teste de hipótese descrito no capitulo 5 , que compara os modelo Binomial e Beta Binomial via fator de Bayes.

Apresentamos na tabela 7.12 , para vários valores do parâmetro $\omega$, as respectivas estimativas do parâmetro $\xi$, da verossimilhança marginal dada em 5.13 , do fator de Bayes dado em 5,11 e do inverso do fator de Bayes. 
Tabela 7.12 - Fator de Bayes considerando vários valores de $\omega$ para os dados genéticos.

\begin{tabular}{ccccc}
\hline$\omega$ & $\hat{\xi}$ & $I_{H_{1}}$ & $\hat{B}(\omega)$ & $\hat{B}(\omega)^{-1}$ \\
\hline 0,05 & 0,5809 & $4,0425 \times 10^{-192}$ & 0,0532 & 18,811 \\
0,1 & 0,5807 & $8,263 \times 10^{-192}$ & 0,0260 & 38,451 \\
0,15 & 0,5801 & $3,843 \times 10^{-192}$ & 0,0559 & 17,883 \\
0,2 & 0,5793 & $6,432 \times 10^{-193}$ & 0,3341 & 2,9929 \\
0,25 & 0,5784 & $5,2302 \times 10^{-194}$ & 4,1088 & 0,2434 \\
0,3 & 0,5774 & $2,5357 \times 10^{-195}$ & 84,748 & 0,0118 \\
\hline
\end{tabular}

Observamos que para $\omega=0,1$ temos $\hat{B}(\omega)^{-1}$ igual à 38,454 , o que sugere uma forte evidência contra o modelo Binomial. Para todos os valores de $\omega$ testados entre 0,05 e 0,2 , tivemos valores de $\hat{B}(\omega)^{-1}$ maiores do que 3 , obtendo assim evidências favoráveis ao modelo Beta-Binomial. Somente quando $\omega$ é maior do que 0,3 , a hipótese alternativa do modelo BetaBinomial torna-se menos favorável que a distribuição Binomial. Contudo, no geral, concluímos que a probabilidade de associação entre os pares de cromossomos parece variar de núcleo para núcleo, ou seja há evidências para se rejeitar a hipótese $H_{0}: \omega=0$ (distribuição Binomial).

\section{2 - Segunda aplicação: dados de um jogador de basquete}

Estes dados, analisados por Hsiao (1994), se referem ao número de acertos $y_{i}$ num total de $n_{i}$ tentativas durante o i-ésimo jogo do jogador Vinnie Johnson da liga americana de basquete Temos os dados de 380 jogos do jogador, que foram partidas disputadas entre as temporadas de 1985 e 1989.

Numa análise prévia dos dados, observamos que Vinnie Johnson parece ter maior sucesso em alguns jogos do que em outros, o que sugere uma probabilidade de acerto não homogênea de jogo para jogo. Neste caso, um modelo Binomial com uma mesma probabilidade de sucesso, não seria o modelo mais correto para ajustar a variabilidade de jogo para jogo.

Para verificar se a variabilidade extra-Binomial está de fato presente nos dados, assumimos para a variável $Y_{i}$ (número de acertos em $n_{i}$ tentativas durante o i-ésimo jogo), $i=1, \ldots, 380$, os modelos Binomial, Beta-Binomial, Binomial Correlacionado e mistura de duas 
distribuições Binomiais. Os resultados Clássicos e Bayesianos obtidos para cada um desses modelos são dados nas seções 7.2.1 e 7.2.2, respectivamente.

Apresentamos na tabela 7.13 os dados dos 380 jogos do Vinnie Johnson, onde temos o número de arremessos por jogo $\left(n_{t}\right)$, o número de acertos por jogo $\left(y_{t}\right)$ e a frequência que cada par de observação $\left(n_{i}, y_{i}\right)$ ocorre. Por exemplo, o primeiro par de observação, $n_{i}=1$ e $y_{i}=0$, aparece com frequência 1 , indicando que dos 380 jogos em apenas 1 tivemos $n_{i}=1$ e $y_{i}=0$.

Tabela 7.13 - Dados do jogador de basquete Vinnie Johnson.

\begin{tabular}{|c|ccccccccccccccccc|}
\hline $\mathrm{n}_{\mathrm{i}}$ & $\mathrm{I}$ & 3 & 3 & 3 & 4 & 4 & 4 & 5 & 5 & 5 & 6 & 6 & 6 & 6 & 6 & 7 & 7 \\
$\mathrm{y}_{\mathrm{i}}$ & 0 & 0 & 1 & 2 & 0 & 1 & 2 & 0 & 1 & 2 & 0 & 1 & 2 & 3 & 4 & 1 & 2 \\
Freq & 1 & 3 & 2 & 1 & 3 & 3 & 1 & 3 & 6 & 2 & 1 & 3 & 2 & 1 & 1 & 3 & 5 \\
\hline $\mathrm{n}_{\mathrm{i}}$ & 7 & 7 & 8 & 8 & 8 & 8 & 8 & 8 & 9 & 9 & 9 & 9 & 9 & 9 & 10 & 10 & 10 \\
$\mathrm{y}_{\mathrm{i}}$ & 3 & 4 & 1 & 2 & 3 & 4 & 5 & 6 & 1 & 2 & 3 & 4 & 5 & 6 & 2 & 3 & 4 \\
Freq & 6 & 2 & 1 & 8 & 5 & 5 & 1 & 1 & 2 & 4 & 7 & 1 & 4 & 1 & 3 & 10 & 9 \\
\hline $\mathrm{n}_{\mathrm{i}}$ & 10 & 10 & 10 & 10 & 10 & 11 & 11 & 11 & 11 & 11 & 11 & 11 & 11 & 12 & 12 & 12 & 12 \\
$\mathrm{y}_{\mathrm{i}}$ & 5 & 6 & 7 & 8 & 9 & 1 & 2 & 3 & 4 & 5 & 6 & 7 & 8 & 3 & 4 & 5 & 6 \\
Freq & 7 & 3 & 2 & 1 & 1 & 1 & 1 & 6 & 7 & 6 & 6 & 3 & 1 & 3 & 6 & 7 & 5 \\
\hline $\mathrm{n}_{\mathrm{i}}$ & 12 & 13 & 13 & 13 & 13 & 13 & 13 & 13 & 14 & 14 & 14 & 14 & 14 & 14 & 14 & 14 & 15 \\
$\mathrm{y}_{\mathrm{i}}$ & 7 & 3 & 4 & 5 & 6 & 7 & 8 & 9 & 3 & 4 & 5 & 6 & 7 & 8 & 9 & 5 & 6 \\
Freq & 8 & 2 & 4 & 8 & 7 & 7 & 4 & 3 & 1 & 4 & 2 & 10 & 7 & 5 & 2 & 1 & 2 \\
\hline $\mathrm{n}_{\mathrm{i}}$ & 15 & 15 & 15 & 15 & 16 & 16 & 16 & 16 & 16 & 16 & 16 & 16 & 16 & 16 & 17 & 17 & 17 \\
$\mathrm{y}_{\mathrm{i}}$ & 7 & 8 & 9 & 10 & 3 & 4 & 5 & 6 & 7 & 8 & 9 & 10 & 11 & 12 & 6 & 7 & 8 \\
Freq & 10 & 2 & 5 & 1 & 1 & 1 & 2 & 4 & 6 & 7 & 9 & 6 & 3 & 1 & 2 & 3 & 5 \\
\hline $\mathrm{n}_{\mathrm{i}}$ & 17 & 17 & 17 & 17 & 18 & 18 & 18 & 18 & 18 & 19 & 19 & 19 & 19 & 19 & 20 & 20 & 20 \\
$\mathrm{y}_{\mathrm{i}}$ & 9 & 10 & 11 & 6 & 7 & 8 & 9 & 10 & 12 & 8 & 10 & 11 & 12 & 13 & 9 & 10 & 12 \\
Freq & 3 & 5 & 1 & 1 & 3 & 2 & 4 & 3 & 1 & 1 & 2 & 1 & 2 & 1 & 5 & 3 & 2 \\
\hline $\mathrm{n}_{\mathrm{i}}$ & 21 & 21 & 21 & 21 & 21 & 21 & 22 & 22 & 22 & 22 & 23 & 23 & 24 & 25 & 25 & & \\
$\mathrm{y}_{\mathrm{i}}$ & 7 & 8 & 9 & 10 & 11 & 13 & 1 & 2 & 1 & 1 & 2 & 1 & 1 & 1 & 1 & & \\
Freq & 1 & 1 & 1 & 1 & 1 & 2 & 1 & 2 & 1 & 1 & 2 & 1 & 1 & 1 & 1 & & \\
\hline
\end{tabular}

\subsection{1 - Resultados da análise Clássica}

Na tabela 7.14 apresentamos os resultados das análises Clássicas dos modelos Binomial, Beta-Binomial, Binomial Correlacionado e mistura de duas distribuições Binomiais, considerando os dados do jogador Vinnie Johnson. Esses resultados foram obtidos utilizando a metodologia proposta no decorrer da dissertação. 
Tabela 7.14 - Resultados das análises Clássicas dos modelos para os dados do jogador Vinnie Johnson.

\begin{tabular}{cccc}
\hline Modelo & Parâmetro & EMV & IC $(95 \%)$ \\
\hline Binomial & $p$ & 0,4342 & $(0,4201 ; 0,4482)$ \\
\hline Beta & $\alpha$ & 12,1002 & $(6,7261 ; 17,4743)$ \\
Binomial & $\beta$ & 15,9106 & $(8,9067 ; 22,9145)$ \\
\hline Binomial & $\delta$ & 0,0179 & $(0,0067 ; 0,0292)$ \\
Correlacionado & $p$ & 0,4282 & $(0,4120 ; 0,4445)$ \\
\hline Mistura de 2 & $\theta_{1}$ & 0,073 & $(0,0366 ; 0,1093)$ \\
Distribuições & $\theta_{2}$ & 0,4594 & $(0,4444 ; 0,4744)$ \\
Binomiais & $\pi$ & 0,0517 & $(0,0231 ; 0,0803)$ \\
\hline
\end{tabular}

Para discriminar os modelos na análise Clássica, considerando os dados do jogador Vinnie Johnson, utilizamos o critério de mínima informação (AIC), dado na seção 5.1 , e apresentamos os resultados obtidos na tabela 7.15

Tabela 7.15 - Discriminação Clássica dos modelos para os dados do jogador Vinnie Johnson.

\begin{tabular}{cc}
\hline Modelo & AIC \\
\hline Binomial & 1621 \\
Beta-Binomial & 1586,9 \\
Binomial Correlacionado & 1606,3 \\
Mistura de 2 distribuições Binomiais & 1519 \\
\hline
\end{tabular}

De acordo com a tabela 7.15, observamos que os modelos Beta Binomial, Binomial Correlacionado e mistura de duas distribuições Binomiais apresentaram AIC inferiores ao do modelo Binomial, comprovando a presença da variabilidade extra-Binomial nos dados do jogador Vinnie Johnson. Por ter apresentado o menor AIC e com diferenças significativas para os AIC's dos modelos Beta-Binomial e Binomial Correlacionado, o modelo de mistura de duas distribuições Binomiais foi escolhido como o melhor modelo para ajustar esses dados na análise Clássica. 


\subsection{2 - Resultados da análise Bayesiana}

Na tabela 7.16 apresentamos os resultados da análise Bayesiana do modelo Binomial, considerando os dados do jogador Vinnie Johnson. Esses resultados foram obtidos utilizando a metodologia proposta no apêndice 4 deste trabalho.

Tabela 7.16 - Sumários a posteriori do modelo Binomial para os dados do jogador Vinnie Johnson.

\begin{tabular}{cccc}
\hline Parâmetro & Média & DP & Int. Cred. (95\%) \\
\hline$p$ & 0,4342 & 0,007152 & $(0,4202 ; 0,4482)$ \\
\hline
\end{tabular}

Comparando os resultados da tabela 7.16 com os resultados Clássicos do modelo Binomial dados na tabela 7.14 , observamos que as estimativas pontuais e por intervalo do parâmetro $p$ praticamente coincidiram.

$\mathrm{Na}$ análise Bayesiana do modelo Beta-Binomial para os dados do jogador Vinnie Johnson, assumimos as distribuições a priori dadas em (2.15) com $a_{1}=140, b_{1}=12, a_{2}=130 \mathrm{e}$ $b_{2}=8$.

Baseados nas distribuições condicionais a posteriori dadas em (2.18), utilizamos o algoritmo Metropolis-Hastings para a simulação dos parâmetros. Para cada parâmetro geramos 5 cadeias de 12000 pontos, sendo que para cada cadeia descartamos as primeiras 6000 iterações e consideramos as iterações $6080{ }^{\mathrm{a}}, 6160{ }^{\mathrm{a}}, \ldots, 12000^{\mathrm{a}}$, totalizando assim uma amostra final de 375 pontos.

Consideramos os seguintes valores iniciais para as cadeias dos parâmetros $\alpha$ e $\beta$ :

$$
\begin{aligned}
& \alpha=\left[\begin{array}{lllll}
8 & 10 & 12 & 14 & 16
\end{array}\right] \\
& \beta=\left[\begin{array}{lllll}
7 & 11 & 15 & 19 & 23
\end{array}\right]
\end{aligned}
$$

A tabela 7.17 apresenta os resumos a posteriori dos parâmetros $\alpha$ e $\beta$. 
Tabela 7.17 - Sumários a posteriori do modelo Beta-Binomial para os dados do jogador Vinnie Johnson.

\begin{tabular}{cccccc}
\hline Parâmetro & Média & DP & Int. Cred. (95\%) & GR & Tac \\
\hline$\alpha$ & 11,9534 & 0,7141 & $(10,564 ; 13,4898)$ & 1,0003 & 37,66 \\
$\beta$ & 15,7918 & 0,9263 & $(13,7115 ; 17,6235)$ & 1,0003 & 37,05 \\
\hline
\end{tabular}

Comparando os resultados da tabela 7.17 com os resultados Clássicos do modelo BetaBinomial dados na tabela 7.14, observamos que as médias a posteriori de $\alpha$ e $\beta$ ficaram bem próximas dos EMV desses parâmetros, ao passo que os intervalos de credibilidade de $\alpha$ e $\beta$ apresentaram uma precisão maior que os intervalos de confiança, obtidos por aproximação assintótica.

Para ambos os parâmetros tivemos uma razoável taxa de aceitação dos valores gerados pelo algoritmo Metropolis-Hastings e a convergência foi atingida pelo critério de Gelman \& Rubin, pois os valores do fator de redução e escala se aproximaram de 1 .

$\mathrm{Na}$ análise Bayesiana do modelo Binomial Correlacionado para os dados do jogador Vinnie Johnson, assumimos as distribuições a priori dadas em (3.8) com $a=0, b=0,03, c=12$ e $d=15$.

Para a simulação dos parâmetros, utilizamos as distribuições condicionais a posteriori dadas em (3.11). Geramos 5 cadeias de 8000 pontos para cada parâmetro, através do algoritmo Metropolis-Hastings. Para cada cadeia descartamos os 4000 valores iniciais e consideramos as iterações $4040{ }^{\mathrm{a}}, 4080^{\mathrm{a}}, \ldots, 8000^{\mathrm{a}}$, totalizando assim uma amostra final de 500 pontos.

Consideramos os seguintes valores iniciais para as cadeias dos parâmetros $\delta$ e $p$ :

$$
\begin{aligned}
& p=\left[\begin{array}{lllll}
0,4 & 0,42 & 0,44 & 0,46 & 0,48
\end{array}\right] \\
& \delta=\left[\begin{array}{lllll}
0,01 & 0,02 & 0,03 & 0,04 & 0,05
\end{array}\right]
\end{aligned}
$$

A tabela 7.18 apresenta os resumos a posteriori dos parâmetros $p$ e $\delta$.

Tabela 7.18 - Sumários a posteriori do modelo Binomial Correlacionado para os dados do jogador Vinnie Johnson.

\begin{tabular}{cccccc}
\hline Parâmetro & Média & DP & Int. Cred. (95\%) & GR & Tac \\
\hline$p$ & 0,4277 & 0,0079 & $(0,4120 ; 0,4434)$ & 1,0013 & 65,10 \\
$\delta$ & 0,0184 & 0,0051 & $(0,0085 ; 0,028)$ & 1,0000 & 63,35 \\
\hline
\end{tabular}


Comparando os resultados da tabela $7.18 \mathrm{com}$ os resultados Clássicos do modelo Binomial Correlacionado dados na tabela 7.14, observamos que os estimadores pontuais e por intervalo ficaram bem próximos. Outra caracteristica interessante é que apesar do EMV e da média posteriori do paràmetro $\delta$ apresentarem valores próximos de 0 , os intervalos de confiança e de credibilidade para este parâmetro não contém o número 0 , o que é uma forte evidência que existe uma correlação positiva entre as variáveis binárias de $Y$,

Para os parâmetros $\delta$ e $p$ tivemos uma excelente taxa de aceitação dos valores gerados pelo algoritmo Metropolis-Hastings e a convergência foi atingida pelo critério de Gelman \& Rubin, pois os valores do fator de redução e escala se aproximaram de 1 .

Na analise Bayesiana do modelo de mistura de duas distribuições Binomiais para os dados do jogador Vinnie Johnson, assumimos as distribuições a priori dadas em (4.10) com $a_{1}=2, b_{1}=28, a_{2}=30, b_{2}=35, c=1$ e $d=20$.

Baseados nas distribuições condicionais a posteriori dadas em (4.13), utilizamos o algoritmo Gibbs Sampling para a simulação dos parâmetros. Para cada parâmetro geramos 5 cadeias de 10000 pontos, sendo que para cada cadeia descartamos as primeiras 5000 iterações e consideramos as iterações $5050{ }^{a}, 5100{ }^{a}, \ldots ., 10000^{a}$, totalizando assim uma amostra final de 500 pontos.

Consideramos os seguintes valores iniciais para as cadeias dos parâmetros $\theta_{1}, \theta_{2}$ e $\pi$ :

$$
\begin{aligned}
& \theta_{1}=\left[\begin{array}{lllll}
0,1 & 0,3 & 0,5 & 0,7 & 0,9
\end{array}\right] \\
& \theta_{2}=\left[\begin{array}{lllll}
0,1 & 0,3 & 0,5 & 0,7 & 0,9
\end{array}\right] \\
& \pi=\left[\begin{array}{lllll}
0,1 & 0,3 & 0,5 & 0,7 & 0,9
\end{array}\right]
\end{aligned}
$$

A tabela 7.19 apresenta os resumos a posteriori dos parâmetros $\theta_{1}, \theta_{2}$ e $\pi$.

Tabela 7.19 - Sumários a posteriori do modelo de mistura de duas distribuições Binomiais para os dados do jogador Vinnie Johnson.

\begin{tabular}{ccccc}
\hline Parâmetro & Média & DP & Int. Cred. (95\%) & GR \\
\hline$\theta_{1}$ & 0,0717 & 0,0173 & $(0,0439 ; 0,1085)$ & 1,0031 \\
$\theta_{2}$ & 0,459 & 0,0076 & $(0,4443 ; 0,4738)$ & 1,0018 \\
$\pi$ & 0,0515 & 0,0144 & $(0,0288 ; 0,0831)$ & 1,0044 \\
\hline
\end{tabular}


Comparando os resultados da tabela $7.19 \mathrm{com}$ os resultados Clássicos do modelo de mistura de duas distribuições Binomiais dados na tabela 7.14, observamos que os estimadores pontuais e por intervalo de $\theta_{1}, \theta_{2}$ e $\pi$ ficaram bem próximos.

Para os parâmetros $\theta_{1}, \theta_{2}$ e $\pi$ a convergência foi atingida pelo critério de Gelman \& Rubin, pois os valores do fator de redução e escala se aproximaram de 1 .

Para discriminar os modelos na análise Bayesiana considerando os dados do jogador Vinnie Johnson, encontramos o valor exato da verossimilhança marginal do modelo Binomial e estimativas de Monte Carlo das verossimilhanças marginais dos modelos Beta-Binomial, Binomial Correlacionado e mistura de duas distribuições Binomiais, utilizando a metodologia proposta na seção 5.2 desta dissertação. Apresentamos os resultados obtidos na tabela 7.20.

Tabela 7.20 - Discriminação Bayesiana dos modelos para os dados do jogador Vinnie Johnson.

\begin{tabular}{cc}
\hline Modelo & Estimativa da Ver. Marginal \\
\hline Binomial & $4,836 \times 10^{-354}$ \\
Beta-Binomial & $1,291 \times 10^{-344}$ \\
Binomial Correlacionado & $6,316 \times 10^{-349}$ \\
Mistura de 2 distribuições Binomiais & $1,028 \times 10^{-329}$ \\
\hline
\end{tabular}

Observando a tabela 7.20, verificamos que na análise Bayesiana o modelo de mistura de duas distribuições Binomiais foi o que melhor se ajustou aos dados do jogador Vinnie Johnson, comprovando os resultados Clássicos. O modelo Binomial, como no exemplo anterior, foi o modelo menos eficiente no ajuste dos dados, o que reforça a importância de utilizar em algumas aplicações modelos que possam ajustar a variação extra-Binomial.

\section{3 - Terceira aplicação: um exemplo com covariáveis}

Consideramos o experimento analisado por Crowder (1978), onde uma quantidade de sementes é colocada numa placa coberta com um extrato numa dada diluição. Os números de sementes que germinaram e não germinaram são anotados. Foram considerados dois tipos de 
sementes (O. Aegyptiaca 75 e O. Aegyptiaca 73), dois tipos de extratos (feijão e vagem) e algumas réplicas para as combinações.

Apresentamos os dados desse experimento na tabela 7.21. Para cada réplica, temos o número total de sementes $\left(n_{i}\right)$, o número de sementes que germinaram $\left(y_{i}\right)$ e a proporção de sementes que germinaram $\left(y_{i} / n_{i}\right)$.

Tabela 7.21 - Dados (Cowder (1978))

\begin{tabular}{|ccc|ccc|ccc|ccc|}
\hline \multicolumn{6}{|c|}{ (I) O. Aegyptiaca 75 } & \multicolumn{5}{c|}{ (II) O. Aegyptiaca 73 } \\
\hline \multicolumn{3}{|c|}{ Feijão } & \multicolumn{3}{|c|}{ Vagem } & \multicolumn{3}{c|}{ Feijão } & \multicolumn{3}{c|}{ Vagem } \\
\hline$n_{i}$ & $y_{i}$ & $y_{i} / n_{i}$ & $n_{i}$ & $y_{i}$ & $y_{i} / n_{i}$ & $n_{i}$ & $y_{i}$ & $y_{i} / n_{i}$ & $n_{i}$ & $y_{i}$ & $y_{i} / n_{i}$ \\
39 & 10 & 0,26 & 6 & 5 & 0,83 & 16 & 8 & 0,50 & 12 & 3 & 0,25 \\
62 & 23 & 0,37 & 74 & 53 & 0,72 & 30 & 10 & 0,33 & 41 & 22 & 0,54 \\
81 & 23 & 0,28 & 72 & 55 & 0,76 & 28 & 8 & 0,29 & 30 & 15 & 0,50 \\
51 & 26 & 0,51 & 51 & 32 & 0,63 & 45 & 23 & 0,51 & 51 & 32 & 0,63 \\
39 & 17 & 0,44 & 79 & 46 & 0,58 & 4 & 0 & 0 & 7 & 3 & 0,43 \\
& & & 13 & 10 & 0,77 & & & & & & \\
\hline
\end{tabular}

Numa inspeção dos dados da tabela 7.21 , observamos uma significativa heterogeneidade entre as proporções das réplicas e consideramos na análise dos dados a presença de duas covariáveis $W_{1 i}$ e $W_{2 i}$. Definimos que $W_{1 i}$ assume os valores -1 para o tipo $\mathrm{O}$. Aegyptiaca 75 e 1 para o tipo $\mathrm{O}$. Aegyptiaca 73 e $W_{2 i}$ assume os valores -1 para o extrato de feijão e 1 para o extrato de vagem.

Assumimos para a variável $Y_{i}$ (número de sementes que germinaram em $n_{i}$ sementes), os modelos discutidos no capítulo 6 desta dissertação onde,

$$
p_{i}=\frac{e^{\beta_{0}+\beta_{1} W_{1 i}+\beta_{2} W_{21}+\beta_{3} W_{1 i} W_{2 i}}}{1+e^{\beta_{0}+\beta_{1} W_{1 i}+\beta_{2} W_{2 i}+\beta_{3} W_{1 i} W_{2 i}}} \quad, \text { para } i=1, \ldots, 21
$$

Os resultados obtidos assumindo os modelos de Regressão Logística, Binomial Correlacionado com correlações homogêneas e Binomial Correlacionado com correlações diferentes, são dados nas seções 7.3.1, 7.3.2 e 7.3.3, respectivamente. $\mathrm{Na}$ seção 7.3 .4 apresentamos uma discriminação dos modelos, com base nos critérios discutidos no capítulo 5 deste trabalho. 


\subsection{1 - Aplicação do modelo de Regressão Logística}

Na tabela 7.22 apresentamos os resultados da análise Clássica do modelo de regressão Logistica, para os dados da tabela 7.21. Esses resultados foram obtidos utilizando a metodologia proposta na seção 6.3 .1 deste trabalho.

Tabela 7,22 - Resultados da análise Clássica do modelo de Regressão Logística para os dados da tabela 7.21.

\begin{tabular}{ccc}
\hline Parâmetro & EMV & Int. Conf. (95\%) \\
\hline$\beta_{0}$ & $-0,0206$ & $(-0,1708 ; 0,1296)$ \\
$\beta_{1}$ & $-0,1216$ & $(-0,2718 ; 0,0286)$ \\
$\beta_{2}$ & 0,4646 & $(0,3144 ; 0,6148)$ \\
$\beta_{3}$ & $-0,1945$ & $(-0,3447 ;-0,0443)$ \\
\hline
\end{tabular}

$\mathrm{Na}$ análise Bayesiana do modelo de regressão Logística para os dados da tabela 7.21 , assumimos as distribuições a priori dadas em (6.15), com $\mu_{0}=0,05, \sigma_{0}^{2}=0,2, \mu_{1}=-0,2$, $\sigma_{1}^{2}=0,2, \mu_{2}=0,3, \sigma_{2}^{2}=0,2, \mu_{3}=-0,3$ e $\sigma_{3}^{2}=0,2$.

Para a simulação dos parâmetros, utilizamos as distribuições condicionais a posteriori dadas em (6.18). Geramos 5 cadeias de 8000 pontos para cada parâmetro, através do algoritmo Metropolis-Hastings. Para cada cadeia descartamos os 4000 valores iniciais e consideramos as iterações $4020{ }^{\mathrm{a}}, 4040{ }^{\mathrm{a}}, \ldots ., 8000^{\mathrm{a}}$, totalizando assim uma amostra final de 1000 pontos.

Consideramos os seguintes valores iniciais para as cadeias dos parâmetros $\beta_{0}, \beta_{1}, \beta_{2} \mathrm{e}$ $\beta_{3}:$

$$
\begin{aligned}
& \beta_{0}=\left[\begin{array}{lllll}
0,2 & 0,4 & 0,6 & 0,8 & 1,0
\end{array}\right] \\
& \beta_{1}=\left[\begin{array}{lllll}
0,2 & 0,4 & 0,6 & 0,8 & 1,0
\end{array}\right] \\
& \beta_{2}=\left[\begin{array}{lllll}
0,2 & 0,4 & 0,6 & 0,8 & 1,0
\end{array}\right] \\
& \beta_{3}=\left[\begin{array}{lllll}
0,2 & 0,4 & 0,6 & 0,8 & 1,0
\end{array}\right]
\end{aligned}
$$

Apresentamos na tabela 7.23 os resultados obtidos na análise Bayesiana do modelo de Regressão Logística. 
Tabela 7.23 - Sumários a posteriori do modelo de Regressão Logística para os dados da tabela 7.21 .

\begin{tabular}{cccccc}
\hline Parâmetro & Média & DP & Int. Cred. (95\%) & GR & Tac \\
\hline$\beta_{0}$ & $-0,0123$ & 0,0726 & $(-0,1603 ; 0,1208)$ & 1,0001 & 39,37 \\
$\beta_{1}$ & $-0,1321$ & 0,0725 & $(-0,2780 ; 0,0178)$ & 1,0002 & 38,76 \\
$\beta_{2}$ & 0,4409 & 0,0701 & $(0,3039 ; 0,5845)$ & 1,0002 & 32,13 \\
$\beta_{3}$ & $-0,2156$ & 0,0725 & $(-0,3556 ;-0,0783)$ & 1,0001 & 37,78 \\
\hline
\end{tabular}

Comparando os resultados da tabela $7.23 \mathrm{com}$ os resultados da análise Clássica do modelo de Regressão Logística dados na tabela 7.22 , verificamos que não existe diferenças significativas entre as estimativas pontuais e por intervalo dos parâmetros $\beta_{0}, \beta_{1}, \beta_{2}$ e $\beta_{3}$.

Observamos que os intervalos de credibilidade para os parâmetros $\beta_{2}$ e $\beta_{3}$ não contem o número 0 . Assim, na análise Bayesiana a covariável $W_{2 i}$ (tipo de extrato) e a iteração $W_{1 i} W_{3 i}$ (tipo de semente $\mathrm{x}$ tipo de extrato) tem efeitos significativos na contagem das sementes germinadas.

Para os parâmetros $\beta_{0}, \beta_{1}, \beta_{2}$ e $\beta_{3}$ tivemos uma razoável taxa de aceitação dos valores gerados pelo algoritmo Metropolis-Hastings e a convergência foi atingida pelo critério de Gelman \& Rubin, pois os valores do fator de redução e escala se aproximaram de 1 .

Nas figuras $3.15,3.16,3.17$ e 3.18 apresentamos as trajetórias das cadeias ao longo das iterações dos parâmetros e nas figuras $3.19,3.20,3.21$ e 3.22 apresentamos aproximações das densidades a posteriori marginais de $\beta_{0}, \beta_{1}, \beta_{2}$ e $\beta_{3}$.

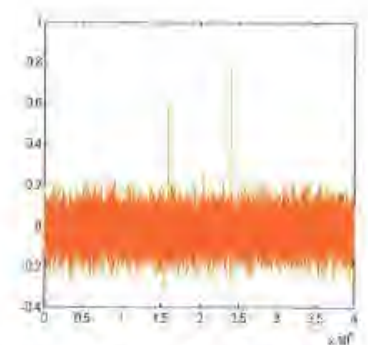

Figura 3.15 - Iterações para $\beta_{i}$

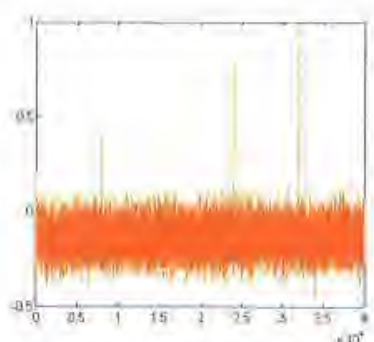

Figura 3.16 - Iterações para $\beta_{1}$ 


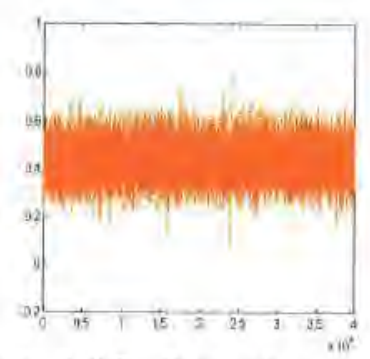

Figura 3.17 - Iterações para $\beta_{2}$

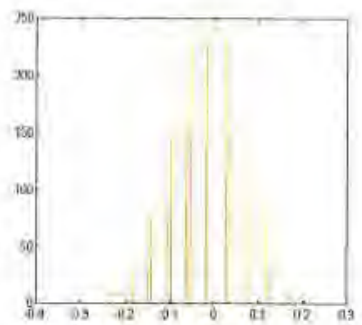

Figura 3.19 - Aproximação da densidade a posteriori marginal de $\beta_{0}$

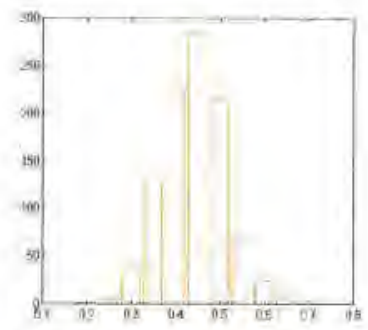

Figura 3.21 - Aproximação da densidade a posteriori marginal de $\beta_{2}$

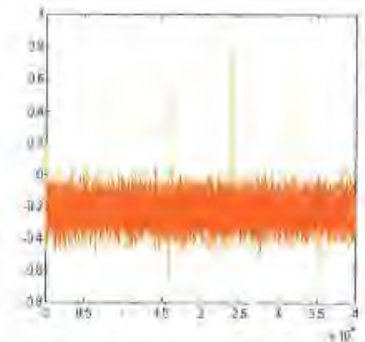

Figura 3.18 - Iterações para $\beta_{3}$

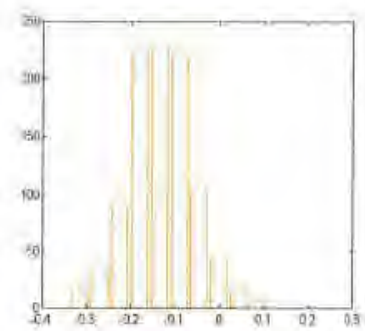

Figura 3.20 - Aproximação da densidade a posteriori marginal de $\beta_{1}$

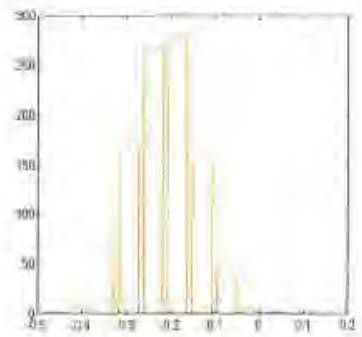

Figura 3.22 - Aproximação da densidade a posteriori marginal de $\beta_{3}$

\subsection{2 - Aplicação do modelo Binomial Correlacionado com correlaçôes homogêneas}

Na tabela 7.24 apresentamos os resultados da análise Clássica do modelo Binomial Correlacionado com correlações homogêneas, para os dados da tabela 7.21. Esses resultados foram obtidos utilizando a metodologia proposta na seção 6.3 .1 deste trabalho. 
Tabela 7.24 - Resultados da análise Clássica do modelo Binomial Correlacionado com correlações homogêneas para os dados da tabela 7.21.

\begin{tabular}{ccc}
\hline Parâmetro & EMV & Int. Conf. $(95 \%)$ \\
\hline$\beta_{0}$ & $-0,0562$ & $(-0,2410 ; 0,1286)$ \\
$\beta_{1}$ & $-0,1557$ & $(-0,3422 ; 0,0307)$ \\
$\beta_{2}$ & 0,4214 & $(0,2279 ; 0,6150)$ \\
$\beta_{3}$ & $-0,2003$ & $(-0,3843 ;-0,0163)$ \\
$\delta$ & 0,02085 & $(0,0033 ; 0,0384)$ \\
\hline
\end{tabular}

Na análise Bayesiana do modelo Binomial Correlacionado com correlações homogêneas para os dados da tabela 7.21, assumimos as distribuições a priori dadas na seção 6.3 .2 com $\mu_{0}=-0,1, \quad \sigma_{0}^{2}=0,2, \quad \mu_{1}=-0,3, \quad \sigma_{1}^{2}=0,2, \quad \mu_{2}=0,45, \quad \sigma_{2}^{2}=0,2, \quad \mu_{3}=-0,1, \quad \sigma_{3}^{2}=0,2$, $a=0,01$ e $b=0,03$.

Baseado nas distribuiçōes condicionais a posteriori dadas na seção 6.3 .2 , utilizamos o algoritmo Metropolis-Hastings para a simulação dos parâmetros. O processo de seleção das amostras foi o mesmo utilizado para o modelo de regressão Logística, descrito na seção anterior .

Consideramos os seguintes valores iniciais para as cadeias dos parâmetros $\beta_{0}, \beta_{1}, \beta_{2}$, $\beta_{3}$ e $\delta$ :

$$
\begin{aligned}
& \beta_{0}=\left[\begin{array}{lllll}
-0,5 & -0,3 & -0,1 & 0,1 & 0,3
\end{array}\right] \\
& \beta_{1}=\left[\begin{array}{lllll}
-0,5 & -0,3 & -0,1 & 0,1 & 0,3
\end{array}\right] \\
& \beta_{2}=\left[\begin{array}{lllll}
0,2 & 0,4 & 0,6 & 0,8 & 1,0
\end{array}\right] \\
& \beta_{3}=\left[\begin{array}{lllll}
-0,6 & -0,4 & -0,2 & 0 & 0,2
\end{array}\right] \\
& \delta=\left[\begin{array}{lllll}
0 & 0,01 & 0,02 & 0,03 & 0,04
\end{array}\right]
\end{aligned}
$$

Apresentamos na tabela 7.25 os resultados obtidos na análise Bayesiana do modelo Binomial Correlacionado com correlaçōes homogêneas. 
Tabela 7.25 - Sumários a posteriori do modelo Binomial Correlacionado com correlações homogêneas para os dados da tabela 7.21.

\begin{tabular}{cccccc}
\hline Parâmetro & Média & DP & Int. Cred. (95\%) & GR & Tac \\
\hline$\beta_{0}$ & $-0,0618$ & 0,0726 & $(-0,2172 ; 0,0854)$ & 1,0008 & 41,85 \\
$\beta_{1}$ & $-0,1754$ & 0,0801 & $(-0,3359 ;-0,0144)$ & 1,0007 & 35,51 \\
$\beta_{2}$ & 0,4395 & 0,0802 & $(0,2848 ; 0,6034)$ & 1,0000 & 42,68 \\
$\beta_{3}$ & $-0,1788$ & 0,0789 & $(-0,329 ;-0,0320)$ & 1,0001 & 39,84 \\
$\delta$ & 0,0203 & 0,0053 & $(0,0109 ; 0,0293)$ & 1,0000 & 82,94 \\
\hline
\end{tabular}

Comparando os resultados da tabela 7.25 com os resultados da análise Clássica do modelo Binomial Correlacionado dados na tabela 7.24, observamos que não existe diferenças significativas entre as estimativas pontuais e por intervalo dos parâmetros $\beta_{0}, \beta_{1}, \beta_{2}, \beta_{3}$ e $\delta$.

Observamos que os intervalos de credibilidade para os parâmetros $\beta_{1}, \beta_{2}, \beta_{3}$ e $\delta$ não contém o número 0. Assim, na análise Bayesiana as covariáveis $W_{1}$ (tipo de semente) e $W_{2 i}$ (tipo de extrato), a iteração $W_{1} W_{2 i}$ (tipo de semente x tipo de extrato) e a correlação $\delta$ tem efeitos significativos na contagem das sementes germinadas.

Para os parâmetros $\beta_{0}, \beta_{1}, \beta_{2}, \beta_{3}$ e $\delta$ tivemos uma razoável taxa de aceitação dos valores gerados pelo algoritmo Metropolis-Hastings e a convergência foi atingida pelo critério de Gelman \& Rubin, pois os valores do fator de redução e escala se aproximaram de 1.

Nas figuras $3.23,3.24,3.25,3.26$ e 3.27 apresentamos as trajetórias das cadeias ao longo das iterações dos parâmetros e nas figuras $3.28,3.29,3.30,3.31$ e 3.32 apresentamos aproximações das densidades a posteriori marginais de $\beta_{0}, \beta_{1}, \beta_{2}, \beta_{3}$ e $\delta$.

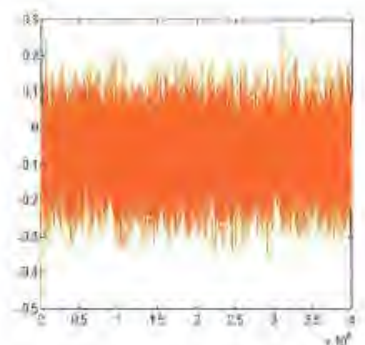

Figura 3.23 - Iterações para $\beta_{0}$

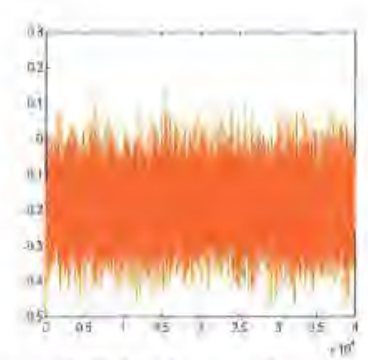

Figura 3.24 - Iterações para $\beta_{1}$ 


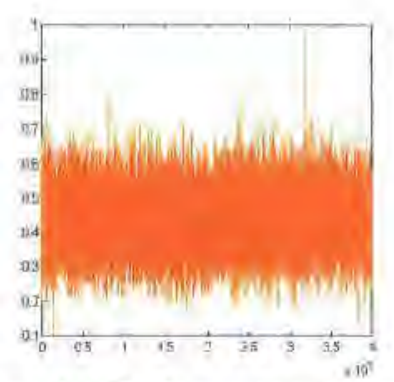

Figura 3.25 - Iterações para $\beta_{2}$

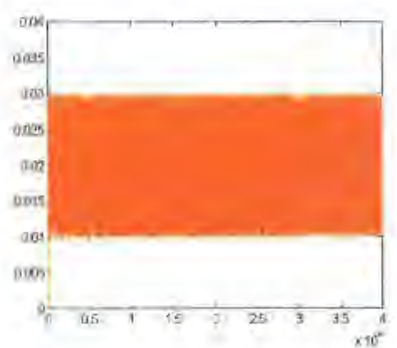

Figura 3.27 - Iterações para $\delta$

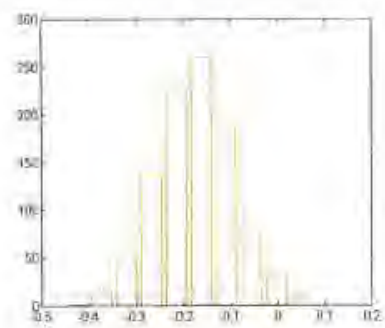

Figura 3.29 - Aproximação da densidade a posteriori marginal de $\boldsymbol{\beta}_{1}$

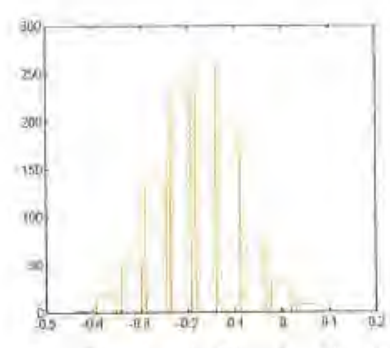

Figura 3.31 - Aproximação da densidade a posteriori marginal de $\beta_{3}$

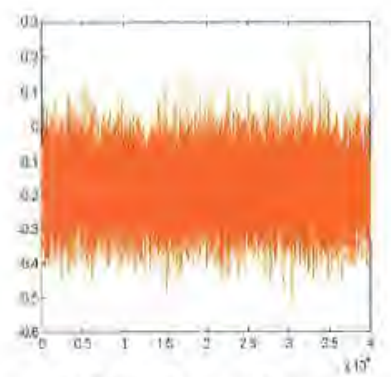

Figura 3.26 - Iterações para $\beta_{3}$

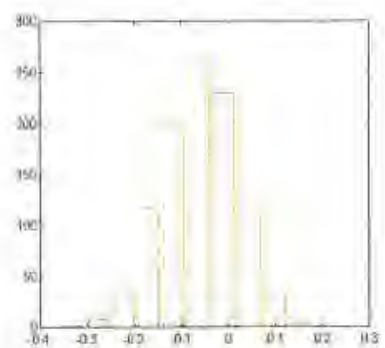

Figura 3.28 - Aproximação da densidade a posteriori marginal de $\beta_{0}$

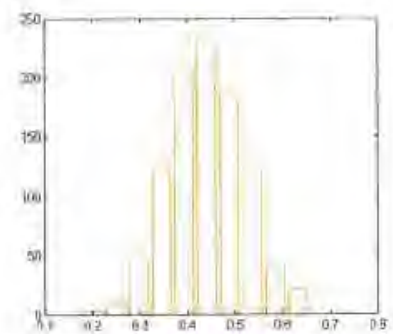

Figura 3.30 - Aproximação de densidade a posteriori marginal de $\beta_{2}$

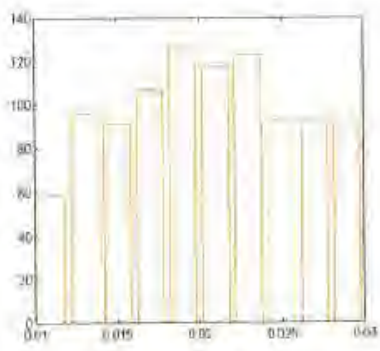

Figura 3.32 - Aproximação de densidade a posteriori marginal de $\delta$ 


\subsection{3 - Aplicação do modelo Binomial Correlacionado com correlações diferentes}

A análise Bayesiana do modelo Binomial Correlacionado com correlações diferentes para os dados da tabela 7.21, foi feita de maneira similar a que apresentamos na seção anterior para o modelo Binomial Correlacionado com correlações homogêneas, considerando os mesmos valores para $\mu_{0}, \sigma_{0}^{2}, \mu_{1}, \sigma_{1}^{2}, \mu_{2}, \sigma_{2}^{2}, \mu_{3}, \sigma_{3}^{2}, a$ e $b$, as mesmas condições iniciais para as cadeias dos parâmetros e o mesmo processo de seleção das amostras geradas.

$\mathrm{Na}$ tabela 7.26 apresentamos os resumos a posteriori para os parâmetros $\beta_{0}, \beta_{1}, \beta_{2}, \beta_{3}$ e $\delta_{1}, i=1, \ldots, 21$.

Tabela 7.26 - Sumarios a posteriori do modelo Binomial Correlacionado com correlações diferentes para os dados da tabela 7.21 .

\begin{tabular}{|c|c|c|c|c|c|}
\hline Parâmetro & Média & DP & Int. Cred. $(95 \%)$ & GR & Tac \\
\hline$\beta_{0}$ & $-0,0620$ & 0,0815 & $(-0,2151 ; 0,0926)$ & 1,0003 & 0,4285 \\
\hline$\beta_{1}$ & $-0,1748$ & 0,0798 & $(-0,3304 ;-0,0204)$ & 0,9989 & 0,3634 \\
\hline$\beta_{2}$ & 0,4405 & 0,0791 & $(0,2819 ; 0,5888)$ & 1,0035 & 0,4328 \\
\hline$\beta_{3}$ & $-0,1724$ & 0,0793 & $(-0,3277 ;-0,0169)$ & 1,0009 & 0,3996 \\
\hline$\delta_{1}$ & 0,0203 & 0,0054 & $(0,0106 ; 0,0294)$ & 0,9990 & 0,8433 \\
\hline$\delta_{2}$ & 0,0201 & 0,0055 & $(0,0106 ; 0,0296)$ & 0,9995 & 0,8343 \\
\hline$\delta_{3}$ & 0,0203 & 0,0053 & $(0,0107 ; 0,0293)$ & 0,9983 & 0,8400 \\
\hline$\delta_{4}$ & 0,0200 & 0,0052 & $(0,0107 ; 0,0289)$ & 0,9985 & 0,8394 \\
\hline$\delta_{5}$ & 0,0201 & 0,0053 & $(0,0108 ; 0,0290)$ & 0,9989 & 0,8371 \\
\hline$\delta_{6}$ & 0,0202 & 0,0051 & $(0,0110 ; 0,0293)$ & 0,9995 & 0,8387 \\
\hline$\delta_{7}$ & 0,0198 & 0,0053 & $(0,0105 ; 0,0293)$ & 0,9997 & 0,8299 \\
\hline$\delta_{8}$ & 0,0201 & 0,0054 & $(0,0107 ; 0,0296)$ & 0,9995 & 0,8365 \\
\hline$\delta_{n}$ & 0,0200 & 0,0050 & $(0,0108 ; 0,0292)$ & 0,9989 & 0,8404 \\
\hline$\delta_{10}$ & 0,0200 & 0,0054 & $(0,0106 ; 0,0293)$ & 0,9986 & 0,8389 \\
\hline$\delta_{11}$ & 0,0200 & 0,0053 & $(0,0106 ; 0,0292)$ & 0,9982 & 0,8372 \\
\hline$\delta_{12}$ & 0,0202 & 0,0052 & $(0,0107 ; 0,0292)$ & 1,0027 & 0,8401 \\
\hline$\delta_{13}$ & 0,0200 & 0,0052 & $(0,0107 ; 0,0291)$ & 0,9980 & 0,8348 \\
\hline$\delta_{14}$ & 0,0202 & 0,0053 & $(0,0109 ; 0,0293)$ & 1,0004 & 0,8401 \\
\hline$\delta_{15}$ & 0,0203 & 0,0053 & $(0,0107 ; 0,0293)$ & 0,9991 & 0,8383 \\
\hline$\delta_{16}$ & 0,0202 & 0,0052 & $(0,0111 ; 0,0292)$ & 0,9987 & 0,8402 \\
\hline$\delta_{17}$ & 0,0202 & 0,0053 & $(0,0108 ; 0,0294)$ & 0,9985 & 0,8376 \\
\hline$\delta_{18}$ & 0,0200 & 0,0052 & $(0,0111 ; 0,0292)$ & 1,0032 & 0,8385 \\
\hline$\delta_{19}$ & 0,0201 & 0,0052 & $(0,0108 ; 0,0292)$ & 0,9981 & 0,8409 \\
\hline$\delta_{20}$ & 0,0205 & 0,0052 & $(0,0107 ; 0,0293)$ & 0,9987 & 0,8380 \\
\hline$\delta_{21}$ & 0,0200 & 0,0054 & $(0,0107 ; 0,0291)$ & 0,9999 & 0,8397 \\
\hline
\end{tabular}


Observamos que as estimativas Bayesianas para os parâmetros $\beta_{0}, \beta_{1}, \beta_{2}, \beta_{3}$ dadas na tabela 7.26, se aproximaram das estimativas dadas na tabela 7.25 , referentes ao modelo com correlações homogêneas e as correlações $\delta_{i}, i=1, \ldots, 21$, apresentaram médias e intervalos de credibilidade bem próximas das estimativas de $\delta$ dadas na tabela 7.25.

Para todos os parâmetros tivemos uma razoável taxa de aceitação dos valores gerados pelo algoritmo Metropolis-Hastings e a convergência foi atingida pelo critério de Gelman \& Rubin, pois os valores do fator de redução e escala se aproximaram de 1

\subsection{4 - Comparação dos modelos}

Para uma discriminação Clássica dos modelos de regressão Logística e Binomial Correlacionado com correlações homogêneas, utilizamos o critério de minima informação (AIC), discutido na seção 5.1.

Apresentamos na tabela 7.27 os resultados do AIC obtidos com os dados da tabela 7.21.

Tabela 7.27 - Discriminação Clássica dos modelos para os dados da tabela 7.21 .

\begin{tabular}{cc}
\hline Modelo & AIC \\
\hline Regressão Logística & 117,874 \\
Binomial Correlacionado & 114,851 \\
\hline
\end{tabular}

Observamos que o AIC do modelo de Binomial Correlacionado é menor que o AIC do modelo de Regressão Logística, indicando que a correlação $\delta$ entre as variáveis binárias de $Y_{i}$ é um importante fator para melhorar o ajuste dos dados da tabela 7.21, quando trabalhamos com covariáveis.

Para discriminar os modelos na análise Bayesiana considerando os dados da tabela 7.21, encontramos estimativas de Monte Carlo das verossimilhanças marginais dos modelos de Regressão Logística, Binomial Correlacionado com correlações homogêneas e Binomial Correlacionado com correlações diferentes, utilizando as expressões dadas em $(6.22),(6.23)$ e (6.24), respectivamente. Na tabela 7.28 apresentamos os resultados obtidos 
Tabela 7.28 - Discriminação Bayesiana dos modelos para os dados da tabela 7.21

\begin{tabular}{cc}
\hline Modelo & Estimativa da Ver. Marginal \\
\hline Regressão Logística & $4,0612 \times 10^{-25}$ \\
Binomial Correlacionado com correl. Homog & $4,3538 \times 10^{-24}$ \\
Binomial Correlacionado com correl. Difer. & $4,8834 \times 10^{-24}$ \\
\hline
\end{tabular}

Observamos que as estimativas de Monte Carlo da verossimilhança para os dois modelos que englobam com correlação, ficaram bem proximas e ambas superaram a estimativa do modelo de regressão Logística. Portanto, concluímos que os dados analisados por Crowder (1978), apresentam uma variabilidade extra-Binomial significativa. 


\section{Capítulo 8: Conclusões e Considerações Futuras}

Nesta dissertação, comprovamos através dos exemplos numéricos analisados, que os modelos usados para ajustar a variabilidade extra-Binomial são fundamentais em muitas aplicações onde temos dados de contagens de sucessos dos ensaios de um experimento. Nos três exemplos discutidos no capítulo 7 , a suposição Binomial usual produz um ajuste ineficiente dos dados, e o uso de modelos para explicar a variação extra-Binomial torna-se necessário, para os dados com ou sem a presença de covariáveis.

$\mathrm{O}$ uso do modelo de misturas de distribuições Binomiais com a introdução das variáveis não observáveis (latentes), é uma alternativa eficiente para ajustar dados de contagens de sucessos em diversos experimentos.

A análise Bayesiana dos modelos usados para ajustar a variabilidade extra-Binomial, permite incorporar aos modelos, informações prévias a respeito dos parâmetros, e isso muitas vezes produz inferências mais precisas. As técnicas de simulação de Monte Carlo em Cadeias de Markov (MCMC), são apropriadas para a obtenção de sumários a posteriori de interesse dos parâmetros, pois possuem fácil implementação e não exigem um conhecimento computacional avançado.

Em relação as propostas futuras, podemos considerar a análise Bayesiana de modelos mais complexos, como modelos de misturas de várias $(>2)$ distribuições Binomiais, e estudar modelos para super dispersão envolvendo não apenas a variabilidade extra-Binomial, mais a super dispersão de outros modelos, como por exemplo os modelos de Poisson. 


\section{Apêndice A}

\section{Algoritmo Gibbs Sampling}

O algoritmo Gibbs Sampling foi introduzido formalmente por Geman e Geman (1984), que propuseram um esquema de amostragem de distribuições explorando as distribuições condicionais completas, através de um algoritmo iterativo que define uma Cadeia de Markov.

$\mathrm{O}$ algoritmo Gibbs Sampling é essencialmente um esquema iterativo de amostragem de uma cadeia de Markov cujo núcleo de transição é formado pelas distribuições condicionais completas.

\section{Descrição do método:}

Suponha que a distribuição de interesse é $\pi(\theta)$ onde $\theta=\left(\theta_{1}, \theta_{2}, \ldots, \theta_{p}\right)$ e que as distribuições condicionais completas a posteriori $\pi\left(\theta_{i} / \theta_{1}, \theta_{2}, \ldots, \theta_{1-1}, \theta_{i+1}, \ldots, \theta_{p}\right), \quad i=1, \ldots, p$, estão disponiveis sob a forma padronizada. Podemos então gerar amostras através do seguinte procedimento:

Passo 1: iniciar o contador das iterações, $j=1$, e atribuir valores iniciais ${\underline{\theta^{(0)}}}^{(0)}\left(\theta_{1}^{(0)}, \theta_{2}^{(0)}, \ldots, \theta_{p}^{(0)}\right)$.

Passo 2: obter um novo valor $\theta^{(j)}=\left(\theta_{1}^{(j)}, \theta_{2}^{(j)}, \ldots, \theta_{p}^{(j)}\right)$ através de sucessivas geração de valores:

$$
\begin{gathered}
\theta_{1}^{(\jmath)} \sim \pi\left(\theta_{1} / \theta_{2}^{(j-1)}, \ldots, \theta_{p}^{(\jmath-1)}\right) \\
\theta_{2}^{(\jmath)} \sim \pi\left(\theta_{2} / \theta_{1}^{(\jmath)}, \theta_{3}^{(\jmath-1)}, \ldots, \theta_{p}^{(j-1)}\right) \\
\vdots \\
\theta_{p}^{(\jmath)} \sim \pi\left(\theta_{p} / \theta_{1}^{(\jmath)}, \theta_{2}^{(\jmath)}, \ldots, \theta_{p-1}^{(\jmath)}\right)
\end{gathered}
$$


Passo 3: mudamos o contador $j$ para $j+1$ e retornamos a (ii) até a convergência.

Geman e Geman (1984) mostram que os valores gerados para $\theta_{i}, i=1, \ldots, p$, convergem em distribuição para uma variável aleatória da distribuição $\pi(\theta) \operatorname{com} j \rightarrow \infty$. 


\section{Apêndice B}

\section{$\underline{\text { Algoritmo Metropolis-Hastings }}$}

Quando as distribuições condicionais a posteriori $\pi\left(\theta_{i} / \theta_{1}, \theta_{2}, \ldots, \theta_{i-1}, \theta_{i+1}, \ldots, \theta_{p}\right)$, $i=1, \ldots, p$, não apresentam formas de distribuições conhecidas (Normal, Beta, etc.), uma alternativa é o uso do algoritmo Metropolis-Hastings.

A idéia básica é escrever a distribuição condicional a posteriori como o produto de duas funções:

$$
\pi\left(\theta_{i} / \theta_{1}, \theta_{2}, \ldots, \theta_{i-1}, \theta_{i+1}, \ldots, \theta_{p}\right) \propto \psi\left(\theta_{i}\right) \pi\left(\theta_{i}\right)
$$

onde $\pi\left(\theta_{i}\right)$ será usada como núcleo de transição para gerar as amostras de $\theta_{i}$ e tem a forma de uma distribuição padrão. A função $\psi\left(\theta_{i}\right)$ é utilizada no teste de aceitação e rejeição dos valores gerados.

Descrição do método:

Passo 1 : iniciar o contador das iterações, $j=1$, e atribuir valores iniciais $\theta^{(0)}=\left(\theta_{1}^{(0)}, \theta_{2}^{(0)}, \ldots, \theta_{p}^{(0)}\right)$.

Passo 2: obter os novos valores $\theta_{i}^{j}$ a partir da função de transição $\pi\left(\theta_{i}^{j}\right)$.

Passo3: calcular a probabilidade de aceitação do novo valor onde:

$$
\alpha\left(\theta_{i}^{j}\right)=\left\{\begin{array}{c}
\min \left\{1, \frac{\psi\left(\theta_{i}^{j}\right)}{\psi\left(\theta_{i}^{j-1}\right)}\right\}, \operatorname{se} \psi\left(\theta_{i}^{j}\right)>0 \\
1, \text { caso contrário }
\end{array}\right.
$$

Passo 4: gerar o valor $u$ a partir de uma distribuição Uniforme $(0,1)$ e fazer: 


$$
\theta_{i}^{j}= \begin{cases}\theta_{i}^{j} & \text { se } u \leq \alpha\left(\theta_{i}^{j}\right) \\ \theta_{i}^{j+1} & \text { caso contrário }\end{cases}
$$

Passo 5: mudamos o contador $j$ para $j+1$ e retornamos a (ii) até a convergência. 


\section{Apêndice C \\ Critério de convergência de Gelman e Rubin}

Uma forma simples de verificar convergência é a utilização de várias cadeias em paralelo começando com diferentes pontos iniciais. Com isso, evita-se que as cadeias se concentrem em regiões em torno de modas locais.

A inspeção visual de similaridade entre as trajetórias das várias cadeias após um certo número de iterações é um forte indício de convergência. Gelman \& Rubin (1992) formalizaram essa idéia, de que as trajetórias das cadeias devem ser a mesma depois de convergirem, através do uso de técnicas de análise de variância. A idéia geral é testar se a dispersão intra-cadeias é maior do que a dispersão inter-cadeias. Isto equivale a dizer que: o histograma das cadeias como um todo deve ser similar aos histogramas das cadeias tomadas individualmente.

Considerando $m$ cadeias que evoluem em paralelo e uma função real $t(\theta)$, tem-se $m$ trajetórias $\left\{t_{i}^{(1)}, t_{i}^{(2)}, \ldots, t_{i}^{(n)}\right\}, i=1, \ldots, m$, para $t$. Portanto, podem ser obtidas a variância entre as cadeias, $B$, e a variância dentro das cadeias, $W$. As fórmulas correspondentes são dadas por:

$$
B=\frac{n}{m-1} \sum_{i=1}^{m}\left(\bar{t}_{i}-\bar{t}\right)^{2} \quad \text { e } \quad W=\frac{1}{m(n-1)} \sum_{i=1}^{m} \sum_{j=1}^{n}\left(t_{i}^{(j)}-\bar{t}_{t}\right)^{2}
$$

onde $\bar{t}_{i}$ é a média das observações da cadeia $i$ e $\bar{t}$ é a média dessas médias, $i=1, \ldots, m$.

A variância de $t$ pode ser estimada de forma não viciada por:

$$
\hat{V}(t(\theta))=\left(1-\frac{1}{n}\right) W+\left(\frac{1}{n}\right) B
$$

Se as cadeias ainda não estiverem convergindo, então essa estimativa é maior que $V(t(\theta))$ pois os valores iniciais ainda estão sendo influenciados pelos valores dos outros parâmetros da cadeia, de forma que a distribuição de equilibrio ainda não foi atingida, indicando que eles foram 
escolhidos com dispersão maior que a da distribuição de equilíbrio. Por outro lado, $W$ fornece estimativas menores que $V(t(\theta))$, pois a cadeia não terá coberto toda a variabilidade de $t(\theta)$

Um indicador de convergência é dado pela chamada redução potencial estimada da escala $\hat{R}=\sqrt{\hat{V}(t(\theta)) / W}$. A medida que $n$ cresce, ambos os estimadores acabarão convergindo para $V(t(\theta))$ e $R$ convergirá para 1 . Assim, $\hat{R}$ pode ser usado como indicador de convergência pela avaliação de sua proximidade a 1. Gelman (1995) sugere aceitar como garantia de convergência valores de $R \leq 1,1$. 


\section{Referências Bibliográficas}

1) ALTHAM, P.M.E. Two Generalizations of the Binomial Distribution. Applied Statistics, n.27, p. 162-167,1978.

2) BOX, G.E.P.; JENKINS, G.M.; REINSEL, G.C. Time Series Analysis: Forecasting and Control, third edition, 1994.

3) CHIB, S.; GREENBERG, E. Understanding the Metropolis-Hastings. The American Statistican, n.49, p.327-335, 1995.

4) CROWDER, M.J. Beta-Binomial Anova for Proportions. Applied Statistics, n.27, p.34-37, 1978 .

5) GELFAND, A.E.; SMITH, A.F.M. Sampling Based Approaches to Calculating Marginal Densities. Journal of the American Statistical Association, n. 85, p.398 - 409, 1990.

6) GELMAN, A.E.; GELMAN, D. Stochastic Relaxation, Gibbs Distributions and the Bayesian Restoration of Images. IEEE Transactions on Pattern Analysis and Machine Intelligence, n.6, p. 721-741, 1984.

7) GELMAN, A.E.; RUBIN, D. Inference from Iterative Simulation Using Multiple Sequences. Statistical Sciences, n.7, p.457-472, 1992.

8) GRIFFITHS, D.A. Maximum Likelihood Estimation for the Beta-Binomial Distribuition and an Application to the Household Distribution of the Total Number of Cases of a Diease. Biometrics, n.29, p.637-648, 1973.

9) HSIAO, C.K. Bayesian Tests of Extra-Binomial Variability with Emphasis on the Boundary Case, PhD thesis, Carnegie-Mellon University, U.S.A., 1994. 


\section{Apêndice D}

\section{Análise Clássica e Bayesiana do modelo Binomial}

Considerando independentes as contagens de sucessos $y_{1}, y_{2}, \ldots, y_{N}$, a função de verossimilhança para o modelo Binomial, utilizando a expressão (1.1), é dada por,

$$
L(p)=\prod_{i=1}^{N}\left[\left(\begin{array}{l}
n_{i} \\
y_{i}
\end{array}\right) p^{y_{i}}(1-p)^{n_{i}-y_{i}}\right]=\left[\prod_{i=1}^{N}\left(\begin{array}{l}
n_{i} \\
y_{i}
\end{array}\right)\right] p^{\sum_{i=1}^{N} y_{i}}(1-p) \sum_{i=1}^{N}\left(n_{i}-y_{i}\right)
$$

Aplicando o logaritmo na expressão acima temos,

$$
\begin{aligned}
& l(p)=\log [L(p)]=\log \left\{\left[\prod_{i=1}^{N}\left(\begin{array}{l}
n_{i} \\
y_{i}
\end{array}\right)\right] p^{\sum_{i=1}^{N} y_{i}}(1-p) \sum_{i=1}^{N}\left(n_{i}-y_{i}\right)\right\} \\
& =\sum_{i=1}^{N}\left[\log \left(\begin{array}{l}
n_{i} \\
y_{i}
\end{array}\right)\right]+\log (p) \sum_{i=1}^{N} y_{i}+\log (1-p) \sum_{i=1}^{N}\left(n_{i}-y_{i}\right)
\end{aligned}
$$

A derivada de primeira ordem do logaritmo da função de verossimilhança em relação ao parâmetro $p$ é dada por,

$$
\frac{\partial l(p)}{\partial p}=\frac{\sum_{i=1}^{N} y_{i}}{p}-\frac{\sum_{i=1}^{N}\left(n_{i}-y_{t}\right)}{1-p}
$$

Ao contrário dos outros modelos discutidos nesta dissertação, no modelo Binomial temos 
que $\frac{\partial l(p)}{\partial p}=0$ é uma equação linear em relação a $p$. Assim, determinamos o valor exato do EMV para $p$, dado por,

$$
\hat{p}=\frac{\sum_{i=1}^{N} y_{i}}{\sum_{i=1}^{N} n_{i}}
$$

$\mathrm{Na}$ análise Bayesiana do modelo Binomial, assumimos para a probabilidade de sucesso $p$ uma distribuição a priori Uniforme $(0,1)$. Essa distribuição foi usada no capítulo 5 para encontrar a verossimilhança marginal exata do modelo. Utilizando esta verossimilhança marginal, podemos encontrar a densidade a posteriori exata para o parâmetro $p$, dada por,

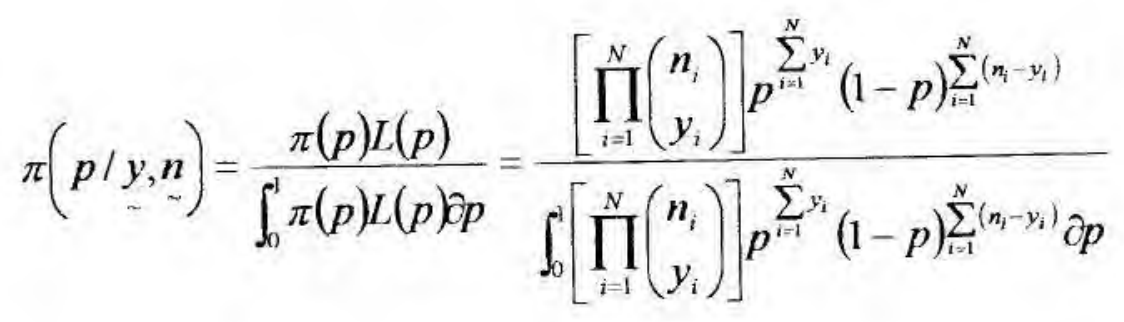

$$
\begin{aligned}
& =\frac{\Gamma\left(\sum_{i=1}^{N} n_{t}+2\right)}{\Gamma\left(\sum_{i=1}^{N} y_{i}+1\right) \Gamma\left(\sum_{i=1}^{N}\left(n_{i}-y_{i}\right)+1\right)} p^{\sum_{i=1}^{N} y_{i}}(1-p)^{\sum_{i=1}^{N}\left(n_{i}-y_{i}\right)}
\end{aligned}
$$

Algumas características do parâmetro $p$ podem ser determinadas a partir da expressão acima, sem o uso de métodos numéricos ou computacionais. A seguir apresentamos o desenvolvimento matemático para obter a esperança e a variância a posteriori de $p$. 


\section{1) Esperança:}

$$
\begin{aligned}
& E(p / y, n)=\int_{0}^{1} p \pi(p / \underset{\sim}{y, n}) \partial p \\
& =\int_{0}^{1} p \frac{\Gamma\left(\sum_{i=1}^{N} n_{i}+2\right)}{\Gamma\left(\sum_{i=1}^{N} y_{i}+1\right)\left(\sum_{i=1}^{N}\left(n_{i}-y_{i}\right)+1\right)} p^{\sum_{i=1}^{N} y_{i}}(1-p) \sum_{i=1}^{N}\left(n_{i}-y_{i}\right) \partial p \\
& =\frac{\Gamma\left(\sum_{i=1}^{N} n_{i}+2\right)}{\Gamma\left(\sum_{i=1}^{N} y_{i}+1\right)\left(\sum_{i=1}^{N}\left(n_{i}-y_{i}\right)+1\right)} \frac{\Gamma\left(\sum_{i=1}^{N} y_{i}+2\right)\left(\sum_{i=1}^{N}\left(n_{i}-y_{i}\right)+1\right)}{\Gamma\left(\sum_{i=1}^{N} n_{i}+3\right)} \\
& =\frac{\left(\sum_{i=1}^{N} n_{i}+1\right) !\left(\sum_{i=1}^{N} y_{i}+1\right) !}{\left(\sum_{i=1}^{N} y_{i}\right) !\left(\sum_{i=1}^{N} n_{i}+2\right) !}=\frac{\sum_{i=1}^{N} y_{i}+1}{\sum_{i=1}^{N} n_{i}+2}
\end{aligned}
$$

2) Variância:

$$
\begin{aligned}
& {[E(p / y, n)]^{2}=\frac{\left(\sum_{i=1}^{N} y_{i}+1\right)^{2}}{\left(\sum_{i=1}^{N} n_{i}+2\right)^{2}}} \\
& E\left[(p / \underset{\sim}{y, n})_{\sim}^{2}\right]=\int_{0}^{1} p^{2} \pi(p / \underset{\sim}{y, n}) \partial p
\end{aligned}
$$




$$
\begin{aligned}
& =\int_{0}^{1} p^{2} \frac{\Gamma\left(\sum_{i=1}^{N} n_{i}+2\right)}{\Gamma\left(\sum_{i=1}^{N} y_{i}+1\right)\left(\sum_{i=1}^{N}\left(n_{i}-y_{i}\right)+1\right)} p^{\sum_{i=1}^{N} y_{i}}(1-p) \sum_{i=1}^{N}\left(n_{1}-y_{i}\right) \partial p \\
& =\frac{\Gamma\left(\sum_{i=1}^{N} n_{i}+2\right)}{\Gamma\left(\sum_{i=1}^{N} y_{i}+1\right)\left(\sum_{i=1}^{N}\left(n_{i}-y_{i}\right)+1\right)} \frac{\Gamma\left(\sum_{i=1}^{N} y_{i}+3\right)\left(\sum_{i=1}^{N}\left(n_{i}-y_{i}\right)+1\right)}{\Gamma\left(\sum_{i=1}^{N} n_{i}+4\right)} \\
& =\frac{\left(\sum_{i=1}^{N} n_{i}+1\right) !\left(\sum_{i=1}^{N} y_{i}+2\right) !}{\left(\sum_{i=1}^{N} y_{i}\right) !\left(\sum_{i=1}^{N} n_{i}+3\right) !}=\frac{\left(\sum_{i=1}^{N} y_{i}+2\right)\left(\sum_{i=1}^{N} y_{i}+1\right)}{\left(\sum_{i=1}^{N} n_{i}+3\right)\left(\sum_{i=1}^{N} n_{i}+2\right)}
\end{aligned}
$$

Assim,

$$
\begin{aligned}
& \operatorname{Var}(p / \underline{\sim}, \underline{\sim})=E\left[(p / \underline{\sim}, n)^{2}\right]-[E(p / \underset{\sim}{y, n})]^{2} \\
& =\frac{\left(\sum_{i=1}^{N} y_{i}+2\right)\left(\sum_{i=1}^{N} y_{i}+1\right)}{\left(\sum_{i=1}^{N} n_{r}+3\right)\left(\sum_{i=1}^{N} y_{i}+1\right)}-\frac{\left({ }_{i}\right)}{\left(\sum_{i=1}^{N} n_{i}+2\right)^{2}} \\
& =\frac{\left(\sum_{i=1}^{N} y_{i}+1\right)\left(\sum_{i=1}^{N} n_{i}-\sum_{i=1}^{N} y_{i}+1\right)}{\left(\sum_{i=1}^{N} n_{i}+3\right)\left(\sum_{i=1}^{N} n_{i}+2\right)^{2}}
\end{aligned}
$$

93 


\section{Apêndice E \\ Programa Computacional para o modelo Beta-Binomial}

Neste apêndice, apresentamos um dos programas computacionais utilizados neste trabalho. Este programa foi desenvolvido no "software" Matlab e é baseado no algoritmo de simulação Metroplis-Hastings para o modelo Beta-Binomial, considerando os dados genéticos (Skellam (1948) )

\% MCMC para o modelo Beta-Binomial considerando

os dados genéticos

clear

识 vetores de dados

\begin{tabular}{|c|c|c|c|c|c|c|c|c|c|c|c|c|c|c|c|c|c|c|c|}
\hline & 3 & & & & & & & 3 & 3 & 3 & & 3 & 3 & 3 & 3 & 3 & & & \\
\hline 3 & 3 & 3 & 3 & 3 & 33 & 3 & 3 & 3 & 3 & 3 & 3 & 3 & 3 & 3 & 3 & 3 & 3 & 3 & \\
\hline 3 & 3 & 3 & 3 & 3 & 33 & 3 & 3 & 3 & 3 & 3. & 3 & 3 & 3 & 3 & 3 & 3 & 1 & & \\
\hline 3 & 33 & 3 & 3 & 3 & 33 & 3 & 3 & 3 & 3 & 3 & 3 & 3 & 3 & 3 & 3 & 3 & 3 & & \\
\hline 3 & 33 & 3 & 3 & 3 & 33 & 3 & 3 & 3 & 3 & 3 & 3 & 33 & 33 & 3 & 3 & 3 & 3 & & \\
\hline 3 & 3 & 3 & 3 & 3 & 33 & 3 & 3 & 3 & 3 & 3 & 3 & 33 & 33 & 3 & 3 & 3 & 3 & & \\
\hline 3 & 3 & 3 & 3 & 3 & 33 & 3 & 3 & 3 & 3 & 3 & 3 & 3 & 3 & 3 & 3 & 3 & 3 & & \\
\hline 3 & 33 & 3 & 3 & 3 & 33 & 3 & 3 & 3 & 3 & 3 & 3 & 3 & 3 & 3 & 3 & 3 & 3 & & \\
\hline 3 & 33 & 3 & 3 & 3 & 33 & 3 & 3 & 3 & 3 & 3 & 3 & 3 & 3 & 3 & 3 & 3 & & & \\
\hline 3 & 3 & 3 & 3 & 3 & 33 & 3 & 3 & 3 & 3 & 3 & 3 & 3 & 3 & 3 & 3 & 3 & & & \\
\hline 3 & 33 & 3 & 3 & 3 & 33 & 3 & 3 & 3 & 3 & 3 & 3 & 3 & 3 & 3 & 3 & 3 & & & \\
\hline 3 & 33 & 3 & 3 & 3 & 33 & 3 & 3 & 3 & 3 & 3 & 3 & 33 & 33 & 3 & 3 & 3 & & & \\
\hline 3 & 33 & 3 & 3 & 3 & 33 & 3 & 3 & 3 & 3 & 3 & 3 & 3 & 33 & 3 & 3 & 3 & & & \\
\hline 3 & 33 & 3 & 3 & 3 & 33 & 3 & 3 & 3 & 3 & & & & 33 & 3 & 3 & 3 & & & \\
\hline 3 & & & & & & & & & & & & & & & & & & & \\
\hline
\end{tabular}

$y i=\left[\begin{array}{lllllllllllllllllllllll}0 & 0 & 0 & 0 & 0 & 0 & 0 & 0 & 0 & 0 & 0 & 0 & 0 & 0 & 0 & 0 & 0 & 0 & 0 & 0 & 0 & 0 & 0\end{array}\right]$ $\begin{array}{llllllllllllllllllllllllllll}0 & 0 & 0 & 0 & 0 & 0 & 0 & 0 & 0 & 1 & 1 & 1 & 1 & 1 & 1 & 1 & 1 & 1 & 1 & 1 & 1 & 1 & 1 & \ldots\end{array}$

$\begin{array}{lllllllllllllllllllllllllllll}1 & 1 & 1 & 1 & 1 & 1 & 1 & 1 & 1 & 1 & 1 & 1 & 1 & 1 & 1 & 1 & 1 & 1 & 1 & 1 & 1 & 1 & 1 & \ldots\end{array}$

$\begin{array}{llllllllllllllllllllllllll}1 & 1 & 1 & 1 & 1 & 1 & 1 & 1 & 1 & 1 & 1 & 1 & 1 & 1 & 1 & 1 & 1 & 1 & 1 & 1 & 1 & 1 & 1 & \ldots\end{array}$

$\begin{array}{llllllllllllllllllllllll}1 & 1 & 1 & 1 & 1 & 1 & 1 & 1 & 1 & 1 & 1 & 1 & 1 & 1 & 1 & 1 & 1 & 1 & 1 & 1 & 1 & 1 & 1 & \ldots\end{array}$

$\begin{array}{llllllllllllllllllllllllllll}1 & 1 & 1 & 1 & 1 & 1 & 1 & 1 & 1 & 1 & 1 & 1 & 1 & 1 & 1 & 1 & 1 & 1 & 1 & 1 & 2 & 2 & 2 & \ldots\end{array}$

$\begin{array}{llllllllllllllllllllllll}2 & 2 & 2 & 2 & 2 & 2 & 2 & 2 & 2 & 2 & 2 & 2 & 2 & 2 & 2 & 2 & 2 & 2 & 2 & 2 & 2 & 2 & 2 & \cdots\end{array}$

$\begin{array}{lllllllllllllllllllllllll}2 & 2 & 2 & 2 & 2 & 2 & 2 & 2 & 2 & 2 & 2 & 2 & 2 & 2 & 2 & 2 & 2 & 2 & 2 & 2 & 2 & 2 & 2 & \ldots\end{array}$

$\begin{array}{llllllllllllllllllllllll}2 & 2 & 2 & 2 & 2 & 2 & 2 & 2 & 2 & 2 & 2 & 2 & 2 & 2 & 2 & 2 & 2 & 2 & 2 & 2 & 2 & 2 & 2\end{array} \ldots$

$2 \begin{array}{lllllllllllllllllllllll} & 2 & 2 & 2 & 2 & 2 & 2 & 2 & 2 & 2 & 2 & 2 & 2 & 2 & 2 & 2 & 2 & 2 & 2 & 2 & 2 & 2\end{array}$ 


$\begin{array}{lllllllllllllllllllllll}2 & 2 & 2 & 2 & 2 & 2 & 2 & 2 & 2 & 2 & 2 & 2 & 2 & 2 & 2 & 2 & 2 & 2 & 2 & 2 & 2 & 2 & 2 \ldots \\ 2 & 2 & 2 & 2 & 3 & 3 & 3 & 3 & 3 & 3 & 3 & 3 & 3 & 3 & 3 & 3 & 3 & 3 & 3 & 3 & 3 & 3 & 3 \\ 3 & 3 & 3 & 3 & 3 & 3 & 3 & 3 & 3 & 3 & 3 & 3 & 3 & 3 & 3 & 3 & 3 & 3 & 3 & 3 & 3 & 3 & 3 \\ 3 & 3 & 3 & 3 & 3 & 3 & 3 & 3 & 3 & 3 & 3 & 3 & 3 & 3 & 3 & 3 & 3 & 3 & 3 & 3 & 3 & 3 & 3 \\ 3 & 3 & 3 & 3 & 3 & 3 & 3 & 3 & 3 & 3 & 3 & 3 & 3 & 3 & 3] & \ldots\end{array}$

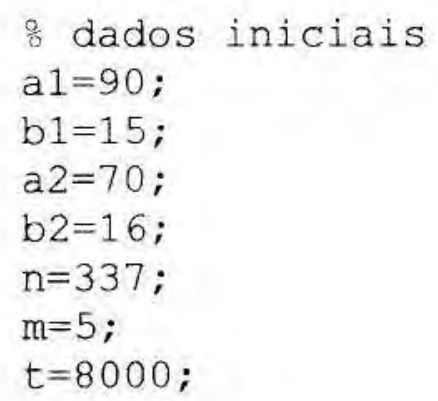

for $j=1: m$

$\operatorname{alf}(1, j)=\operatorname{alfo}(j)$;

$\operatorname{bet}(1, j)=\operatorname{bet} 0(j)$;

for $i=2: t$

$\because$ metropolis hastings 1

$\operatorname{alf}(i, j)=$ gamrnd $(a 1,1 / b 1)$;

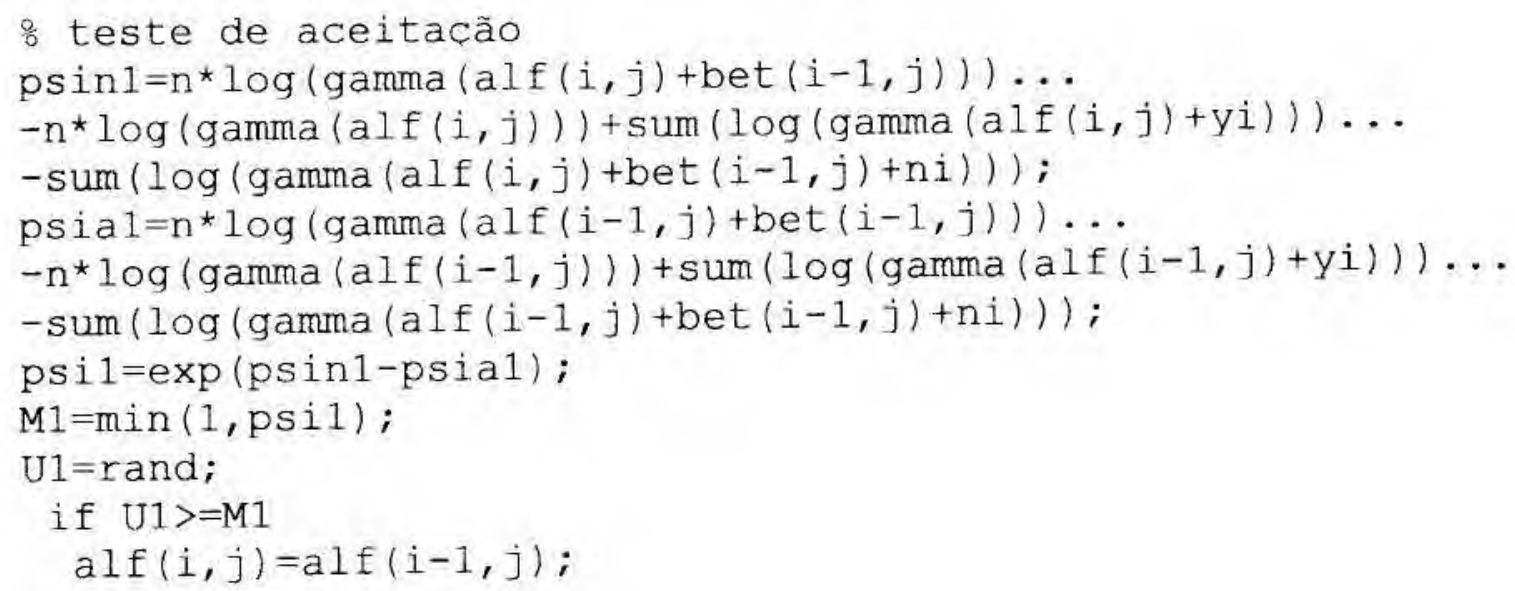


$\operatorname{ir} 1(j)=\operatorname{irl}(j)+1$;

end

\% metropolis hastings 2

$\operatorname{bet}(i, j)=\operatorname{gamrnd}(a 2,1 / b 2)$;

\% teste de aceitacão

$\operatorname{psin} 2=n * \log (\operatorname{gamma}(\operatorname{alf}(i, j)+\operatorname{bet}(i, j))) \ldots$

$-n .{ }^{\star} \log (\operatorname{gamma}(\operatorname{bet}(i, j)))+\operatorname{sum}(\log (\operatorname{gamma}(\operatorname{bet}(i, j)+n i-y i))) \ldots$

$-\operatorname{sum}(\log (\operatorname{gamma}(\operatorname{alf}(i, j)+\operatorname{bet}(i, j)+n i)))$;

psia2 $=n * \log (\operatorname{gamma}(\operatorname{alf}(i, j)+\operatorname{bet}(i-1, j))) \ldots$

$-n{ }^{\star} \log (\operatorname{gamma}(\operatorname{bet}(i-1, j)))+\operatorname{sum}(\log (\operatorname{gamma}(\operatorname{bet}(i-1, j)+n i-y i))) \ldots$

$-\operatorname{sum}(\log (\operatorname{gamma}(\operatorname{alf}(i, j)+\operatorname{bet}(i-1, j)+n i)))$;

psi2 $=\exp (p \sin 2-p s i a 2)$;

M2 $=\min (1, p s i 2)$;

$\mathrm{U} 2=\mathrm{rand}$;

if $\mathrm{U} 2>=\mathrm{M} 2$

$\operatorname{bet}(i, j)=\operatorname{bet}(i-1, j)$;

$\operatorname{ir} 2(j)=i r 2(j)+1$;

end

end

end

\& seleção das amostras

$\mathrm{aaa}=\mathrm{alf}(:)$;

$\mathrm{bbb}=\operatorname{bet}(:)$;

$\mathrm{k}=20$;

alf $00=\operatorname{alf}(4020: k: t,:)$;

bet $00=\operatorname{bet}(4020: k: t,:)$;

alf000=alf00(:);

bet $000=\operatorname{bet} 00(:)$;

\% salvar os dados

save dalfl aaa -ascii;

save dbet $1 \mathrm{bbb}$-ascii;

save dalf2 alf000-ascii;

save dbet 2 bet000-ascii;

¿ inferências

$\operatorname{malf}=\operatorname{mean}(\mathrm{alf} 000)$

mbet $=$ mean $($ bet000) 
sdalf $=\operatorname{std}(a \perp f 000)$

sdbet $=$ std $($ bet 000$)$

\% intervalos de credibilidade

linf alf=prctile (alf000,2.5)

linf bet=prctile (bet000,2.5)

lsup_alf=prctile (alf000,97.5)

1 sup_bet=prctile (bet000,97.5)

: convergência

gralf=gelrub (alf)

grbet=gelrub (bet)

: porcentagem de aceitação

ir $11=1-\operatorname{sum}(\operatorname{ir} 1) / 40000$

ir22=1- sum $(\operatorname{ir} 2) / 40000$

$\because$ verossimilhança marginal

$\mathrm{s}=$ length (alf000);

for $\mathrm{c} 1=1: \mathrm{s}$

for $\mathrm{c}=1: \mathrm{n}$

$\mathrm{hh}(\mathrm{c}, \mathrm{c} 1)=\left(\operatorname{prod}(1: \operatorname{ni}(\mathrm{c})) /\left(\operatorname{prod}(1: \mathrm{yi}(\mathrm{c})){ }^{\star} \operatorname{prod}(1:(\operatorname{ni}(\mathrm{c})-\mathrm{yi}(\mathrm{c})))\right)\right)$

* (gamma (alf000(c1) +bet000(c1)) / (gamma (alf000 (c1))...

* gamma (bet000(c1)) L)*((gamma (alf000 (c1) +yi (c)) ...

* gamma (bet000 (ci) +ni (c) -yi (c))) ...

/(gamma (alf000(c1) +bet000(c1) +ni(c)))) ;

end

end

est_vm=mean (prod (hh))

\% gráficos das iterações

iter alf=plot $(\operatorname{alf}(:))$

iter bet $=$ plot $($ bet $(:))$

\% aproximaçöes das densidades a posteriori marginais

hist_alf=hist (alf000)

hist bet=hist $($ bet 000$)$ 
10) KASS, R.E.; RAFTERY, A.E. Bayes Factors and Model Uncertainty. Journal of the American Statistical Association, n.90, p.773-775, 1995.

11) KLEIN, J.P.; MOESCHBERGER, M.L. Survival Analysis: Techniques for Censored and Truncated Data. Springer-Verlag, New York, 1997.

12) KUPPER, L.L,; HASEMAN, J.K. The Use of a Correlated Binomial Model for the Analysis of Certain Toxicological Experiments. Biometrics, n.34, p.69-76, 1978.

13) LEE, E.T. Statistical Methods for Survival Data Analysis. New York: Wiley, 1992.

14) PRENTICE, R.L. Binary Regression Using an Extended Beta-Binomial Distribution, with Discussion of Correlation Induced by Covariate Measurement Errors. Journal of the American Statistical Association, n.90, p.773-775, 1995.

15) ROBERTS, G.O; SMITH, A.F.M. Bayesian Methods via the Gibbs Sampler and related Markov Chain Monte Carlo Methods. Journal of the Royal Statistical Society, B, n.55, p.3-23, 1993.

16) RUDOLFER, S.,M. A Markov Chain Model of Extra-Binomial Variation. Biometrika, n.77, p. $255-264,1990$.

17) SKELlAM, J.G. A Probability Distribution Derived from the Binomial Distribution by Regarding the Probability of Sucess as Variable between the Sets of Trials. Journal of the Royal Statistical Society, B, p. 257-261, 1948.

18) TANNER, M.A.; WONG, W.H. The Calculation of Posterior Distributions by Data Augmentation (with discussion). Journal of the American Statistical Association, n.82, p.528$550,1995$.

19) TARONE, R.E. Testing the Goodness-of-Fit of the Binomial Distribution. Biometrika, n.66, p. $585-590,1979$. 
20) TIERNEY, L.; KADANE, J.B.; Accurate Approximations for Posterior Moments and Marginal Densities. Journal of the American Statistical Association, n.81, p.82-86, 1986.

21) TITTERINGTON, D.M.; SMITH, A.F.M.; MARKOV, U.E. Statistical Analysis of Finite Mixture Distributions. New York: Wiley, 1985.

22) WILliAMS, D.A. Extra-Binomial Variation in Logistic Linear Models. Applied Statistics, p.31, n.144-148, 1982. 